NISSUNA UMANA INVESTIGAZIONE SI PUO DIMANDARE VERA SCIENZIA S'ESSA NON PASSA PER LE MATEMATICHE DIMOSTRAZIONI LEONARDO DA VINCI

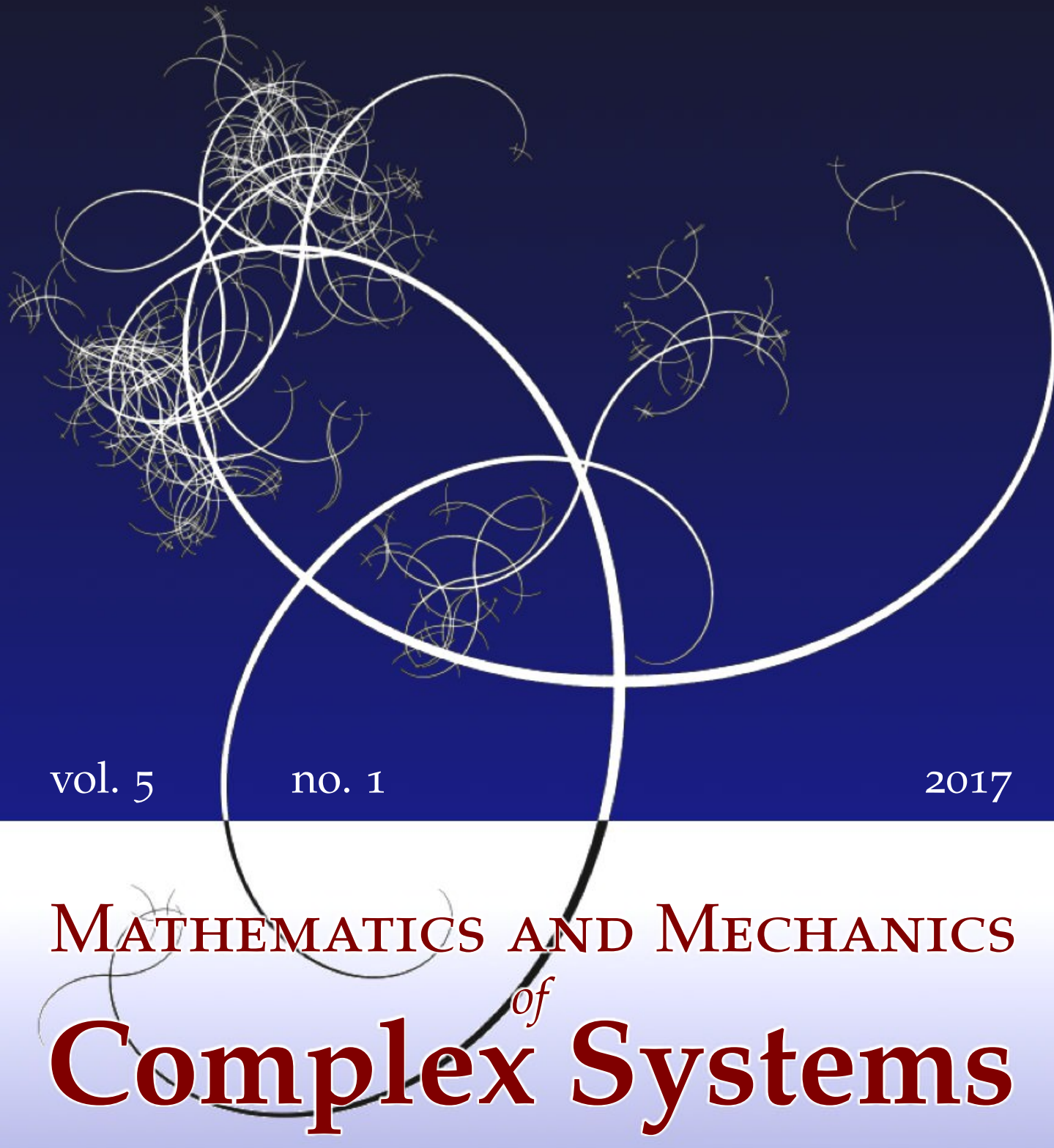

Graeme W. Milton, Marc Briane and Davit Harutyunyan ON THE POSSIBLE EFFECTIVE ELASTICITY TENSORS OF 2-DIMENSIONAL AND 3-DIMENSIONAL PRINTED MATERIALS 


\title{
ON THE POSSIBLE EFFECTIVE ELASTICITY TENSORS OF 2-DIMENSIONAL AND 3-DIMENSIONAL PRINTED MATERIALS
}

\author{
Graeme W. Milton, Marc Briane and Davit Harutyunyan
}

The set $G U_{f}$ of possible effective elastic tensors of composites built from two materials with elasticity tensors $\boldsymbol{C}_{1}>0$ and $\boldsymbol{C}_{2}=0$ comprising the set $U=$ $\left\{\boldsymbol{C}_{1}, \boldsymbol{C}_{2}\right\}$ and mixed in proportions $f$ and $1-f$ is partly characterized. The material with tensor $\boldsymbol{C}_{2}=0$ corresponds to a material which is void. (For technical reasons $\boldsymbol{C}_{2}$ is actually taken to be nonzero and we take the limit $\boldsymbol{C}_{2} \rightarrow 0$ ). Specifically, recalling that $G U_{f}$ is completely characterized through minimums of sums of energies, involving a set of applied strains, and complementary energies, involving a set of applied stresses, we provide descriptions of microgeometries that in appropriate limits achieve the minimums in many cases. In these cases the calculation of the minimum is reduced to a finite-dimensional minimization problem that can be done numerically. Each microgeometry consists of a union of walls in appropriate directions, where the material in the wall is an appropriate $p$-mode material that is easily compliant to $p \leq 5$ independent applied strains, yet supports any stress in the orthogonal space. Thus the material can easily slip in certain directions along the walls. The region outside the walls contains "complementary Avellaneda material", which is a hierarchical laminate that minimizes the sum of complementary energies.

\section{Introduction}

Here we consider what effective elasticity tensors can be produced in the limit $\delta \rightarrow 0$ if we mix in prescribed proportions two materials with positive definite and bounded elasticity tensors $\boldsymbol{C}_{1}$ and $\boldsymbol{C}_{2}=\delta \boldsymbol{C}_{0}$. In the limit $\delta \rightarrow 0$ this represents a mixture of an elastic phase and an extremely compliant phase. Thus we are given a set $U=\left\{\boldsymbol{C}_{1}, \delta \boldsymbol{C}_{0}\right\}$ and we are aiming to characterize as best we can the set $G U_{f}$ of all possible effective tensors of composites having a volume fraction $f$ of phase 1 . The elasticity tensor $\boldsymbol{C}_{1}$ need not be isotropic but if it is anisotropic we require that it has a fixed orientation throughout the composite. Our results are summarized by the theorems in Section 10.

\section{Communicated by Robert P. Lipton.}

MSC2010: 74Q20, 35Q74.

Keywords: printed materials, elastic $G$-closures, metamaterials. 
To get an idea of the enormity of the problem one has to recognize that in three dimensions elasticity tensors can be represented by $6 \times 6$ matrices and these have 21 independent elements. The set of possible elasticity tensors is thus represented as a set in a 21-dimensional space. Even a distorted multidimensional cube in a 21dimensional space needs about 44 million real numbers to represent it (specifying the position in 21-dimensional space of each of the $2^{21}$ vertices). In the case where the two phases are isotropic, one is free to rotate the material to obtain an equivalent structure. Thus the set of possible elasticity tensors is invariant under rotations. As rotations involve three parameters (the Euler angles) this reduces the number of constants needed to describe the elasticity tensor from 21 to $21-3=18$, and thus the elasticity tensor can be represented in an 18-dimensional space of tensor invariants. For example, in the generic case, one can take these 18 invariants as follows: the six eigenvalues of the elasticity tensor; the two independent elements of the normalized eigenstrain associated with the lowest eigenvalue that can be assumed to be diagonal by an appropriate choice of the coordinate axes (which then fixes these axes); the four independent elements of the normalized eigenstrain associated with the second lowest eigenvalue that is orthogonal to the first eigenstrain; the three independent elements of the normalized eigenstrain associated with the third lowest eigenvalue that is orthogonal to the first two eigenstrains; the two independent elements of the normalized eigenstrain associated with the third lowest eigenvalue that is orthogonal to the first three eigenstrains; and the one independent element of the normalized eigenstrain associated with the third lowest eigenvalue that is orthogonal to the first four eigenstrains. This brings the total to $6+2+4+3+2+1=18$. In the same way that it takes two parameters (the bulk and shear moduli) to specify the elastic behavior of an isotropic material, it takes 18 parameters to specify the elastic behavior of a fully anisotropic material.

A distorted cube in this 18-dimensional space still requires about 4.7 million numbers to represent it. This makes exploring the range of possible elasticity tensors a daunting, if not impossible, numerical task. Some numerical exploration of this space has been done by Sigmund [1994; 1995], but we emphasize that this exploration covers only a tiny fraction of the number of possibilities.

Furthermore, the microstructures we found that lie near the boundary of $G U_{f}$ have quite complicated multiscale architectures and thus would be difficult to find numerically. Also, it is not clear whether there are significantly simpler microstructures that can do the job. The numerical route of Sigmund should provide some simpler alternatives for the strut configurations in the multimode structures in the walls, although even then one needs to make subtle multiscale replacements (such as those appearing later in Figures 9 and 10) to achieve the desired performance. Numerical tests need to be made to see whether one can achieve the same performance with simpler structures. While strut configurations might be suitable at low 
volume fractions they are unlikely to be ideal at high volume fractions. Work by Allaire and Aubry [1999] shows that sometimes optimal microstructures necessarily have structure on multiple length scales. Even if one could numerically explore the question, it is not clear how one could summarize the results in a concise way.

From the applied side there is growing interest in trying to characterize the effective elasticity tensors of microstructures that can be produced by 3-dimensional or 2-dimensional printing. A dramatic example of such a microstructure is given in Figure 1. Our results have obvious relevance to this problem in the case where the 3dimensional printed material uses only one isotropic material plus void. Although our microstructures are somewhat extreme, they provide benchmarks that show what is theoretically possible. What is possible in practice will be a subset of this.

The microstructures we consider involve taking three limits. First, as they have structure on multiple length scales, the homogenization limit where the ratio between length scales goes to infinity needs to be taken. Second, the limit $\delta \rightarrow 0$ needs to be taken. Third, as the structure involves thin walls of width $\epsilon$, along which the material can "slip", the limit $\epsilon \rightarrow 0$ needs to be taken so the contribution to the complementary energy of these walls goes to zero, when the structure supports an applied stress. (Here $\epsilon$ should not be confused with the size of the unit cell, as is common in homogenization theory). The limits should be taken in this order, as, for example, standard homogenization theory is justified only if $\delta \neq 0$, so we need to take the homogenization limit before taking the limit $\delta \rightarrow 0$. In the walled structures the material may only occupy a small volume fraction, but this is ultimately irrelevant as the thin walled structures themselves occupy only a very small volume fraction in the final material (which goes to zero as $\epsilon \rightarrow 0$ ).

The case, applicable to printed materials, when phase 2 is actually void, rather than almost void, requires special care. To justify the homogenization steps taken here one has to first replace the void phase 2 with a composite foam having a small amount of phase 1 as the matrix phase, so that its effective elasticity tensor is nonzero, but approaches zero as the proportion of phase 1 in it tends to zero. The microgeometry in this composite needs to be much smaller than the scales in the geometries discussed here, which would involve mixtures of it and phase 1.

We emphasize, too, that our analysis is valid only for linear elasticity, and ignores nonlinear effects such as buckling. In reality the structures will easily buckle under compression. This buckling will occur, for example, in the square beam array structure of Figure 10. Additionally, some of the multimode materials are constructed via a superposition of appropriately shifted and deformed pentamode materials, and these substructures will interact under finite deformations. Also, in practice it would be difficult to realize the delicate multiscale materials that come close to attaining the bounds. Thus what is practically realizable will be just a subset, dependent on the current state of technology, of the set $G U_{f}$. 
While the title refers only to printed materials, the results are also applicable to any periodic, or statistically homogeneous, material containing voids or pores in a homogeneous material. Printed materials are more interesting than typical porous materials as they allow one to explore a wider range of interesting structures.

In a companion paper [Milton et al. 2017] we consider the opposite limit $\delta \rightarrow \infty$, corresponding to a mixture of an elastic material and an almost rigid material.

\section{Review of some bounds on the elastic moduli of two-phase composites and geometries that attain them}

Here we review a selection of results on sharp bounds on the elastic response of twophase composites and the associated problem of identifying optimal geometries that attain them. The interested reader is encouraged to look at the books of NematNasser and Hori [1998], Cherkaev [2000], Milton [2002], Allaire [2002], Torquato [2002] and Tartar [2009], which provide a much more comprehensive survey.

The most elementary bounds on the elastic properties of composites are the classical bounds of Hill [1952], who implicitly showed that

$$
\left\langle[\boldsymbol{C}(\boldsymbol{x})]^{-1}\right\rangle^{-1} \leq \boldsymbol{C}_{*} \leq\langle\boldsymbol{C}(\boldsymbol{x})\rangle .
$$

Here the angular brackets $\langle\cdot\rangle$ denote a volume average, and the inequality holds in the sense of quadratic forms, i.e., for fourth-order tensors $\boldsymbol{A}$ and $\boldsymbol{B}$ satisfying the symmetries of elasticity tensors we say that $\boldsymbol{A} \geq \boldsymbol{B}$ if $\boldsymbol{\epsilon}: \boldsymbol{A \epsilon} \geq \boldsymbol{\epsilon}: \boldsymbol{B} \boldsymbol{\epsilon}$ for all matrices $\boldsymbol{\epsilon}$. While these bounds were not explicitly stated by Hill in his 1952 paper they are an immediate and obvious consequence of his equation (2). If the two phases are isotropic the spectral decomposition of the elasticity tensors $\boldsymbol{C}_{1}$ and $\boldsymbol{C}_{2}$ of the two phases is

$$
\boldsymbol{C}_{1}=3 \kappa_{1} \boldsymbol{\Lambda}_{h}+2 \mu_{1} \boldsymbol{\Lambda}_{s} \quad \text { and } \quad \boldsymbol{C}_{2}=3 \kappa_{2} \boldsymbol{\Lambda}_{h}+2 \mu_{2} \boldsymbol{\Lambda}_{s},
$$

where $\kappa_{1}$ and $\kappa_{2}$ are the bulk moduli of the two phases, $\mu_{1}$ and $\mu_{2}$ are the shear moduli, and

$$
\left\{\boldsymbol{\Lambda}_{h}\right\}_{i j k \ell}=\frac{1}{3} \delta_{i j} \delta_{k \ell}, \quad\left\{\boldsymbol{\Lambda}_{s}\right\}_{i j k \ell}=\frac{1}{2}\left[\delta_{i k} \delta_{j \ell}+\delta_{i \ell} \delta_{k j}\right]-\frac{1}{3} \delta_{i j} \delta_{k \ell}
$$

act as projections. The tensor $\boldsymbol{\Lambda}_{h}$ projects onto the 1-dimensional space of matrices proportional to the second-order identity matrix, while $\boldsymbol{\Lambda}_{s}$ projects onto the 5-dimensional space of trace-free matrices. Similarly if the effective elasticity tensor $\boldsymbol{C}_{*}$ is isotropic we have that $\boldsymbol{C}_{*}=3 \kappa_{1} \boldsymbol{\Lambda}_{h}+2 \mu_{1} \boldsymbol{\Lambda}_{s}$, where $\kappa_{*}$ and $\mu_{*}$ are the effective bulk and shear moduli of the composite. In this paper we are interested in the case where the two phases are well-ordered in the sense that

$$
\boldsymbol{C}_{1} \geq \boldsymbol{C}_{2}
$$


and we will take the limit as $\boldsymbol{C}_{2} \rightarrow 0$, meaning that all the eigenvalues of $\boldsymbol{C}_{2}$ approach zero. In the case of isotropic components this well-ordering assumption is satisfied if $\kappa_{1} \geq \kappa_{2}$ and $\mu_{1} \geq \mu_{2}$, and we will take the limit as $\kappa_{2}, \mu_{2} \rightarrow 0$.

For isotropic composites of two well-ordered materials Hashin and Shtrikman [1963] and Hill [1963] obtained the celebrated bounds

$$
\begin{aligned}
& \kappa_{*} \geq f \kappa_{1}+(1-f) \kappa_{2}-\frac{f(1-f)\left(\kappa_{1}-\kappa_{2}\right)^{2}}{(1-f) \kappa_{1}+f \kappa_{2}+4 \mu_{2} / 3}, \\
& \kappa_{*} \leq f \kappa_{1}+(1-f) \kappa_{2}-\frac{f(1-f)\left(\kappa_{1}-\kappa_{2}\right)^{2}}{(1-f) \kappa_{1}+f \kappa_{2}+4 \mu_{1} / 3}, \\
& \mu_{*} \geq f \mu_{1}+(1-f) \mu_{2}-\frac{f(1-f)\left(\mu_{1}-\mu_{2}\right)^{2}}{(1-f) \mu_{1}+f \mu_{2}+\mu_{2}\left(9 \kappa_{2}+8 \mu_{2}\right) /\left[6\left(\kappa_{2}+2 \mu_{2}\right)\right]}, \\
& \mu_{*} \leq f \mu_{1}+(1-f) \mu_{2}-\frac{f(1-f)\left(\mu_{1}-\mu_{2}\right)^{2}}{(1-f) \mu_{1}+f \mu_{2}+\mu_{1}\left(9 \kappa_{1}+8 \mu_{1}\right) /\left[6\left(\kappa_{1}+2 \mu_{1}\right)\right]} .
\end{aligned}
$$

In fact these bounds (and the variational principles they derive from) hold even if one component has a negative bulk modulus, so long as the composite is stable [Kochmann and Milton 2014]. For 2-dimensional composites (fiber reinforced materials) analogous bounds on the effective elastic moduli were found by Hill [1964] and Hashin [1965]. Bounds on the complex effective bulk and shear moduli of isotropic two-phase 2-dimensional or 3-dimensional composites were also obtained [Gibiansky and Milton 1993; Milton and Berryman 1997; Gibiansky et al. 1993; 1999; Gibiansky and Lakes 1993; 1997]: these are appropriate to the propagation of fixed frequency elastic waves in composites when one or both of the phases is viscoelastic, and when the wavelength is much larger than the microstructure.

An important "attainability principle" is that bounds obtained by substituting a trial field in a variational principle will be attained when the geometry is such that the actual field matches this trial field. This principle was used, for example, in [Milton 1981c] to find geometries that attain the Hashin-Shtrikman bounds on the effective bulk modulus of composites with three or more phases (see also [Gibiansky and Sigmund 2000]). The Hashin-Shtrikman variational principles involve a minimization over trial polarization fields, and the actual polarization field depends on the choice of the elasticity tensor $\boldsymbol{C}_{0}$ of a "reference medium" (typically chosen to be positive definite) and is defined by

$$
\boldsymbol{P}(\boldsymbol{x})=\left(\boldsymbol{C}(\boldsymbol{x})-\boldsymbol{C}_{0}\right) \boldsymbol{\epsilon}(\boldsymbol{x})=\boldsymbol{\sigma}(\boldsymbol{x})-\boldsymbol{C}_{0} \boldsymbol{\epsilon}(\boldsymbol{x}) .
$$

The variational principles require that $\boldsymbol{C}(\boldsymbol{x})-\boldsymbol{C}_{0}$ be either positive semidefinite or negative semidefinite, so in the case of a well-ordered material natural choices of $\boldsymbol{C}_{0}$ are $\boldsymbol{C}_{1}$ or $\boldsymbol{C}_{2}$ and correspondingly the field will be zero in phase 1 or phase 2, respectively. The bounds are obtained by assuming it is constant in the other phase 
(proportional to the identity in case of the bulk modulus bounds, and trace-free for the shear modulus bounds). Hashin and Shtrikman [1963] recognized that the effective bulk modulus would be attained by the Hashin assemblage of coated spheres [Hashin 1962]. A single coated sphere can be a neutral inclusion: if the surrounding "matrix" material has an appropriate bulk modulus (with a specific value between $\kappa_{1}$ and $\kappa_{2}$ ) one can insert it in the matrix material without disturbing a surrounding hydrostatic field (this is the principle behind the unfeelability cloak of Bückmann, Thiel, Kadic, Schittny and Wegener [Bückmann et al. 2014]). The inclusion is invisible to the surrounding field and one can continue to insert similar inclusions, scaled to sizes ranging to the very small, until one essentially obtains a two-phase composite with effective bulk modulus the same as the original matrix material. Due to radial symmetry the forces acting on the spherical inner core will be equally distributed around the boundary and directed radially: thus the field inside the core material is hydrostatic and constant, and hence by the attainability principle, and due to their neutrality, sphere assemblages must attain the effective bulk modulus bounds in (2-5).

One very important class of microgeometries for which the field is constant in one phase are the sequentially layered laminates (first introduced by Maxwell [1873]) built by layering phase 2 with phase 1 in a direction $\boldsymbol{n}_{1}$ (by which we mean $\boldsymbol{n}_{1}$ is perpendicular to the layers), then taking this laminate and layering it again on a much larger length scale with phase 1 in a direction $\boldsymbol{n}_{2}$ to obtain a "rank 2" laminate, and continuing this process until one obtains a rank $m$ laminate, containing in a sense a "core" of phase 2 surrounded by layers of phase 1 . The field is then constant in the core material of phase 2 . An explicit formula for the effective elasticity tensor of such sequentially layered laminates was obtained by Francfort and Murat [1986], generalizing the analogous formulas obtained by Tartar [1985] for conductivity. Of course one can switch the roles of the phases in this construction and thus obtain a material where the field is constant in phase 1. It then immediately follows from the attainability principle (without requiring any calculation!) that one can attain the Hashin-Shtrikman shear modulus bounds (2-5) (and simultaneously the bulk modulus bounds) if one can find a sequentially layered laminate that has an isotropic elasticity tensor, and the easiest way to do this is to do the lamination sequentially by adding infinitesimal layers in random directions. This established the attainability of the Hashin-Shtrikman shear modulus bound [Milton 1986], also established independently and at the same time by Norris [1985], using the differential scheme that was known to be realizable [Milton 1985; Avellaneda 1987a] — in fact Roscoe [1973] had earlier realized the differential approximation scheme could produce the desired shear modulus - and at the same time elegantly by Francfort and Murat [1986], using sequentially layered laminates with just five directions of lamination (in the case of 3-dimensional composites). 
Hill [1963] proved that the bulk modulus bounds are valid also in the non-wellordered case where $\mu_{1} \geq \mu_{2}$ but $\kappa_{1} \leq \kappa_{2}$. As far as we know, the tightest bounds on the effective shear modulus of 3-dimensional composites in the non-well-ordered case where $\mu_{1} \geq \mu_{2}$ but $\kappa_{1} \leq \kappa_{2}$ are those of Milton and Phan-Thien [1982]:

$$
\begin{aligned}
\min _{\substack{\zeta \\
0 \leq \zeta \leq 1}} \frac{8\langle 6 / \mu+7 / \kappa\rangle_{\zeta}+15 / \mu_{2}}{2\left(\langle 21 / \mu+2 / \kappa\rangle_{\zeta} / \mu_{2}+40\langle 1 / \mu\rangle_{\zeta}\langle 1 / \kappa\rangle_{\zeta}\right)} \\
\leq \frac{f(1-f)\left(\mu_{1}-\mu_{2}\right)^{2}}{f \mu_{1}+(1-f) \mu_{2}-\mu_{*}}-(1-f) \mu_{1}-f \mu_{2} \\
\leq \max _{\substack{\zeta \\
0 \leq \zeta \leq 1}} \frac{8 \mu_{1}\langle 6 \kappa+7 \mu\rangle_{\zeta}+15\langle\mu\rangle_{\zeta}\langle\kappa\rangle_{\zeta}}{2\left(\langle 21 \kappa+2 \mu\rangle_{\zeta}+40 \mu_{1}\right)},
\end{aligned}
$$

where for any quantity $a$ taking values $a_{1}$ and $a_{2}$ in phase 1 and phase 2 , respectively, we define $\langle a\rangle_{\zeta} \equiv \zeta a_{1}+(1-\zeta) a_{2}$. These bounds are obtained by eliminating the geometric parameters from the bounds of Milton and Phan-Thien [1982] and are tighter than the better-known Walpole bounds [1966], and are in fact sharp (as they coincide with the Hashin-Shtrikman formula, which corresponds to particular geometries as we have discussed) when the moduli are slightly non-well-ordered. Specifically, the first bound in (2-7) is sharp when the minimum over $\zeta$ is attained at $\zeta=0$, which occurs when

$$
\kappa_{1}-\kappa_{2} \geq-\frac{\left(3 \kappa_{2}+8 \mu_{2}\right)^{2}}{42 \kappa_{2}^{2}} \frac{\kappa_{1} \kappa_{2}}{\mu_{1} \mu_{2}}\left(\mu_{1}-\mu_{2}\right)
$$

and the second bound in (2-7) is sharp when the maximum over $\zeta$ is attained at $\zeta=1$, which occurs when

$$
\kappa_{1}-\kappa_{2} \geq-\frac{\left(3 \kappa_{1}+8 \mu_{1}\right)^{2}}{42 \mu_{1}^{2}}\left(\mu_{1}-\mu_{2}\right)
$$

The bounds (2-5) and (2-7) constrain the pair $\left(\kappa_{*}, \mu_{*}\right)$ to lie in a rectangular box. Berryman and Milton [1988] obtained tighter coupled bounds which slice off two opposing corner regions of the box by eliminating the geometric parameters from the bulk modulus bounds of Beran and Molyneux [1966] (as simplified by Milton [1981b]) and from the shear modulus bounds of Milton and Phan-Thien [1982]. There is good reason to believe these bounds can be improved as the analogous 2-dimensional bounds are not as tight as the bounds of Cherkaev and Gibiansky [1993] coupling $\kappa_{*}$ and $\mu_{*}$, which were derived using the translation method.

For anisotropic composites with an effective tensor $\boldsymbol{C}_{*}$, the microstructure independent bounds that are directly analogous to the Hashin-Shtrikman-Hill bounds, 
given by (2-5), are the "trace bounds"

$$
\begin{aligned}
f \operatorname{Tr}\left[\boldsymbol{\Lambda}_{h}\left(\boldsymbol{C}_{*}-\boldsymbol{C}_{2}\right)^{-1}\right] & \leq \frac{1}{3\left(\kappa_{1}-\kappa_{2}\right)}+\frac{1-f}{3 \kappa_{2}+4 \mu_{2}}, \\
(1-f) \operatorname{Tr}\left[\boldsymbol{\Lambda}_{h}\left(\boldsymbol{C}_{1}-\boldsymbol{C}_{*}\right)^{-1}\right] & \leq \frac{1}{3\left(\kappa_{1}-\kappa_{2}\right)}-\frac{f}{3 \kappa_{1}+4 \mu_{1}}, \\
f \operatorname{Tr}\left[\boldsymbol{\Lambda}_{s}\left(\boldsymbol{C}_{*}-\boldsymbol{C}_{2}\right)^{-1}\right] & \leq \frac{5}{2\left(\mu_{1}-\mu_{2}\right)}+\frac{3\left(\kappa_{2}+2 \mu_{2}\right)(1-f)}{\mu_{2}\left(3 \kappa_{2}+4 \mu_{2}\right)}, \\
(1-f) \operatorname{Tr}\left[\boldsymbol{\Lambda}_{s}\left(\boldsymbol{C}_{1}-\boldsymbol{C}_{*}\right)^{-1}\right] & \leq \frac{5}{2\left(\mu_{1}-\mu_{2}\right)}-\frac{3\left(\kappa_{1}+2 \mu_{1}\right) f}{\mu_{1}\left(3 \kappa_{1}+4 \mu_{1}\right)},
\end{aligned}
$$

obtained independently by Milton and Kohn [1988] and Zhikov [1988; 1991a; 1991b]. In these expressions the fourth-order tensors $\boldsymbol{\Lambda}_{h}$ multiply the fourth-order tensors on their right, and

$$
\operatorname{Tr}[\boldsymbol{A}]=A_{i j i j}
$$

defines the "trace" of a fourth-order tensor (see also [Francfort and Murat 1986] and [Nemat-Nasser and Hori 1993] for related bounds). From the attainability principle it follows that these bounds will be achieved whenever the composite is a sequentially layered laminate, with a core of one phase, surrounded by layers (on widely separated length scales) of the other phase. When $\boldsymbol{C}_{*}$ is isotropic these bounds (2-10) reduce to the Hashin-Shtrikman-Hill bounds (2-5). In the case where the two phases, and hence the composite, are incompressible we can define the five effective shear moduli $\mu_{1}^{*}, \mu_{2}^{*}, \mu_{3}^{*}, \mu_{4}^{*}, \mu_{5}^{*}$ to be the five finite eigenvalues of $\frac{1}{2} \boldsymbol{C}_{*}$, and the second pair of bounds in (2-10) reduce to

$$
\begin{aligned}
\sum_{i=1}^{5} \frac{f}{2\left(\mu_{* i}-\mu_{2}\right)} & \leq \frac{5}{2\left(\mu_{1}-\mu_{2}\right)}+\frac{3\left(\kappa_{2}+2 \mu_{2}\right)(1-f)}{\mu_{2}\left(3 \kappa_{2}+4 \mu_{2}\right)}, \\
\sum_{i=1}^{5} \frac{1-f}{2\left(\mu_{1}-\mu_{* i}\right)} & \leq \frac{5}{2\left(\mu_{1}-\mu_{2}\right)}-\frac{3\left(\kappa_{1}+2 \mu_{1}\right) f}{\mu_{1}\left(3 \kappa_{1}+4 \mu_{1}\right)} .
\end{aligned}
$$

Lipton [1988] established that the analogous bounds for the two effective shear moduli $\mu_{1}^{*}$ and $\mu_{2}^{*}$ of 2-dimensional composites of two incompressible isotropic phases completely characterize $G U_{f}$.

Earlier, Willis [1977] considered anisotropic composites and used the HashinShtrikman variational principle with a trial polarization that was zero in one phase and constant in the other to obtain bounds on the elastic energy of a two-phase composite. He found that these bounds are not microgeometry independent, but rather involve the two-point correlation function, i.e., the probability that a rod with fixed orientation lands with both ends in phase 1 when thrown randomly in a composite. It follows from the attainability principle that the Willis bounds will be 
achieved when the composite is a sequentially layered laminate, with a core of one phase, surrounded by layers (on widely separated length scales) of the other phase.

In a major advance, Avellaneda [1987b] recognized that for any composite of two phases with well-ordered tensors not all the information contained in the twopoint correlation function was relevant to determining the bounds: what was relevant was a "reduced two-point correlation function" that could be represented as a positive measure $\mu(\xi)$ (with unit integral) on the sphere $|\xi|=1$. Roughly speaking one takes the Fourier transform of the two-point correlation function and integrates it over rays $\boldsymbol{k}=k \boldsymbol{\xi}$ in "Fourier space" keeping $\boldsymbol{\xi}$ fixed and integrating over $k$ from 0 to infinity. Most importantly, every such measure could be realized to an arbitrarily high degree of approximation by the measure of a suitable sequentially layered laminate. For example, a measure with weighted delta functions in directions $\xi_{1}$ and $\xi_{2}$ would be realized by a second-rank sequentially layered laminate with layers normal to $\xi_{1}$ and $\xi_{2}$. (We note in passing that these reduced two-point correlation functions of Avellaneda are a special case of the $H$-measures introduced at the same time by Tartar [1989; 1990], in terms of which he could calculate second-order corrections to the effective tensor of a nearly homogeneous composite. $H$-measures were also introduced independently by Gérard [1989; 1994] under the name of microlocal defect measures. For composites of two isotropic phases the HashinShtrikman conductivity bounds, and indeed variational conductivity bounds at any order, can be naturally expressed in terms of the series expansion coefficients of the effective tensor up to a corresponding order for a nearly homogeneous composite, as shown by Milton and McPhedran [1982].)

The fantastic implication was that by summing the Willis bounds [1977], and then minimizing over all positive measures on the sphere, one would get sharp bounds on the sum of elastic complementary energies

$$
W_{f}^{0}\left(\boldsymbol{\sigma}_{1}^{0}, \boldsymbol{\sigma}_{2}^{0}, \boldsymbol{\sigma}_{3}^{0}, \boldsymbol{\sigma}_{4}^{0}, \boldsymbol{\sigma}_{5}^{0}, \boldsymbol{\sigma}_{6}^{0}\right)=\min _{\boldsymbol{C}_{*} \in G U_{f}} \sum_{j=1}^{6} \boldsymbol{\sigma}_{j}^{0}: \boldsymbol{C}_{*}^{-1} \boldsymbol{\sigma}_{j}^{0},
$$

and similarly one could get sharp bounds on the sum of elastic energies

$$
W_{f}^{6}\left(\boldsymbol{\epsilon}_{1}^{0}, \boldsymbol{\epsilon}_{2}^{0}, \boldsymbol{\epsilon}_{3}^{0}, \boldsymbol{\epsilon}_{4}^{0}, \boldsymbol{\epsilon}_{5}^{0}, \boldsymbol{\epsilon}_{6}^{0}\right)=\min _{\boldsymbol{C}_{*} \in G U_{f}} \sum_{i=1}^{6} \boldsymbol{\epsilon}_{i}^{0}: \boldsymbol{C}_{*} \boldsymbol{\epsilon}_{i}^{0} .
$$

Here some of the applied stresses $\boldsymbol{\sigma}_{j}^{0}$ or the applied strains $\boldsymbol{\epsilon}_{i}^{0}$ could be zero. So the evaluation of the functions $W_{f}^{0}\left(\boldsymbol{\sigma}_{1}^{0}, \boldsymbol{\sigma}_{2}^{0}, \boldsymbol{\sigma}_{3}^{0}, \boldsymbol{\sigma}_{4}^{0}, \boldsymbol{\sigma}_{5}^{0}, \boldsymbol{\sigma}_{6}^{0}\right)$ and $W_{f}^{6}\left(\boldsymbol{\epsilon}_{1}^{0}, \boldsymbol{\epsilon}_{2}^{0}, \boldsymbol{\epsilon}_{3}^{0}, \boldsymbol{\epsilon}_{4}^{0}, \boldsymbol{\epsilon}_{5}^{0}, \boldsymbol{\epsilon}_{6}^{0}\right)$ reduces to a finite-dimensional minimization problem which can be done numerically. Hence we will treat the functions $W_{f}^{0}\left(\boldsymbol{\sigma}_{1}^{0}, \boldsymbol{\sigma}_{2}^{0}, \boldsymbol{\sigma}_{3}^{0}, \boldsymbol{\sigma}_{4}^{0}, \boldsymbol{\sigma}_{5}^{0}, \boldsymbol{\sigma}_{6}^{0}\right)$ as being known, and we will call an "Avellaneda material" an associated sequentially layered laminate material with effective tensor $\boldsymbol{C}_{*}=\boldsymbol{C}_{f}^{A}\left(\boldsymbol{\epsilon}_{1}^{0}, \boldsymbol{\epsilon}_{2}^{0}, \boldsymbol{\epsilon}_{3}^{0}, \boldsymbol{\epsilon}_{4}^{0}, \boldsymbol{\epsilon}_{5}^{0}, \boldsymbol{\epsilon}_{6}^{0}\right)$ that 
attains the minimum in (2-14), and similarly we call a "complementary Avellaneda material" an associated sequentially layered laminate material with effective tensor $\boldsymbol{C}_{*}=\widetilde{\boldsymbol{C}}_{f}^{A}\left(\boldsymbol{\sigma}_{1}^{0}, \boldsymbol{\sigma}_{2}^{0}, \boldsymbol{\sigma}_{3}^{0}, \boldsymbol{\sigma}_{4}^{0}, \boldsymbol{\sigma}_{5}^{0}, \boldsymbol{\sigma}_{6}^{0}\right) \in G U_{f}$ that attains the minimum in (2-13). Explicit analytical formulas for the tensors $\boldsymbol{C}_{f}^{A}\left(\boldsymbol{\epsilon}_{1}^{0}, \boldsymbol{\epsilon}_{2}^{0}, \boldsymbol{\epsilon}_{3}^{0}, \boldsymbol{\epsilon}_{4}^{0}, \boldsymbol{\epsilon}_{5}^{0}, \boldsymbol{\epsilon}_{6}^{0}\right)$ and $\widetilde{\boldsymbol{C}}_{f}^{A}\left(\boldsymbol{\sigma}_{1}^{0}, \boldsymbol{\sigma}_{2}^{0}, \boldsymbol{\sigma}_{3}^{0}, \boldsymbol{\sigma}_{4}^{0}, \boldsymbol{\sigma}_{5}^{0}, \boldsymbol{\sigma}_{6}^{0}\right)$ are not generally available, but rather have to be found by numerical computation. When $\boldsymbol{C}_{1} \geq \boldsymbol{C}_{2}$ one needs to take the minimum in (2-13) over the $\boldsymbol{C}_{*}$ of sequentially layered laminates with a core material of phase 2 . Similarly, when $\boldsymbol{C}_{1}^{-1} \geq \boldsymbol{C}_{2}^{-1}$ the minimum in (2-14) also can be taken over the $\boldsymbol{C}_{*}$ of sequentially layered laminates with a core material of phase 2 . We remark that although Avellaneda assumed the tensors $\boldsymbol{C}_{1}$ and $\boldsymbol{C}_{2}$ were isotropic, his analysis easily extends to the case where the tensors are anisotropic but well-ordered (either with $\boldsymbol{C}_{1} \geq \boldsymbol{C}_{2}$ or $\boldsymbol{C}_{2} \geq \boldsymbol{C}_{1}$ ) and with constant orientation throughout the composite: see, for example, Section 23.3 in [Milton 2002].

These $\boldsymbol{C}_{*}$ of sequentially layered laminates are given by the formula of Francfort and Murat [1986] and Gibiansky and Cherkaev [1997b]:

$$
(1-f)\left(\boldsymbol{C}_{1}-\boldsymbol{C}_{*}\right)^{-1}=\left(\boldsymbol{C}_{1}-\boldsymbol{C}_{2}\right)^{-1}-f \sum_{j=1}^{r} c_{j} \boldsymbol{\Gamma}\left(\boldsymbol{n}_{j}\right),
$$

where $r$ is the rank of the sequential laminate, the positive weights $c_{j}$ sum to 1 , the $\boldsymbol{n}_{i}$ are the lamination directions, and $\boldsymbol{\Gamma}(\boldsymbol{n})$ is the fourth-order tensor with elements given by

$$
\begin{aligned}
\{\boldsymbol{\Gamma}(\boldsymbol{n})\}_{h i k \ell}=\frac{1}{4}\left(n_{h}\left\{\boldsymbol{C}(\boldsymbol{n})^{-1}\right\}_{i k} n_{\ell}+\right. & n_{h}\left\{\boldsymbol{C}(\boldsymbol{n})^{-1}\right\}_{i \ell} n_{k} \\
& \left.+n_{i}\left\{\boldsymbol{C}(\boldsymbol{n})^{-1}\right\}_{h k} n_{\ell}+n_{i}\left\{\boldsymbol{C}(\boldsymbol{n})^{-1}\right\}_{h \ell} n_{k}\right),
\end{aligned}
$$

in which $\boldsymbol{C}(\boldsymbol{n})=\boldsymbol{n} \cdot \boldsymbol{C}_{1} \boldsymbol{n}$ is the $3 \times 3$ matrix known as the acoustic tensor, with elements

$$
\{\boldsymbol{C}(\boldsymbol{n})\}_{i k}=\left\{\boldsymbol{n} \cdot \boldsymbol{C}_{1} \boldsymbol{n}\right\}_{i k}=n_{h}\left\{\boldsymbol{C}_{1}\right\}_{h i k \ell} n_{\ell} .
$$

Thus the minimum needs to be taken over the rank $r$ of the sequential laminate, over the positive weights $c_{j}$, which sum to 1 , and over the lamination directions $\boldsymbol{n}_{j}$. In the case where phase 1 is isotropic, with bulk modulus $\kappa_{1}$ and shear modulus $\mu_{1}$, $\boldsymbol{C}(\boldsymbol{n})$ can be easily calculated and one obtains

$\left\{\boldsymbol{\Gamma}\left(\boldsymbol{n}_{j}\right)\right\}_{h i k \ell}$

$=\frac{3 n_{h} n_{i} n_{k} n_{\ell}}{3 \kappa_{1}+4 \mu_{1}}+\frac{1}{4 \mu_{1}}\left(n_{h} \delta_{i k} n_{\ell}+n_{h} \delta_{i \ell} n_{k}+n_{i} \delta_{h k} n_{\ell}+n_{i} \delta_{h \ell} n_{k}-4 n_{h} n_{i} n_{k} n_{\ell}\right)$.

Francfort, Murat, and Tartar [Francfort et al. 1995] proved that when $\boldsymbol{C}_{1}$ is isotropic it suffices to limit attention to laminates of rank $r \leq 6$. When $\boldsymbol{C}_{1}$ is anisotropic we extend an argument due to Avellaneda [1987b]. Consider the set $\mathcal{A}$ consisting of 
all fourth-order tensors $\boldsymbol{A}$ of the form

$$
\boldsymbol{A}=\int_{|\boldsymbol{n}|=1} \boldsymbol{\Gamma}(\boldsymbol{n}) m(d \boldsymbol{n}),
$$

where $m(d \boldsymbol{n})$ is a nonnegative measure on the unit sphere having an integral of 1 over the sphere. Since $\boldsymbol{A}$ satisfies

$$
\{\boldsymbol{A}\}_{h i k \ell}\left\{\boldsymbol{C}_{1}\right\}_{h i k \ell}=\int_{|\boldsymbol{n}|=1}\left\{\boldsymbol{C}(\boldsymbol{n})^{-1}\right\}_{i k}\{\boldsymbol{C}(\boldsymbol{n})\}_{i k} m(d \boldsymbol{n})=3,
$$

it follows that $\mathcal{A}$ is a convex set in a space of dimension $v=20$ (with 20 of the 21 independent matrix elements of $\boldsymbol{A}$ as coordinates, and the remaining element being determined by (2-20)). The extreme points correspond to point masses on the unit sphere. Hence any tensor of the form (2-19) is a convex combination of at most $v+1$ extreme points. Thus the sum (2-15) can be limited to $r \leq 21$; i.e., it suffices to consider laminates up to rank 21. Lipton [1991; 1992; 1994] obtained a complete algebraic characterization of the possible sequentially layered laminates having transverse or orthotropic symmetry and derived explicit expressions for many of the associated bounds. The Avellaneda materials are of course difficult to build in practice since they have structure on multiple length scales. However, if $f$ is small and one phase is void, Bourdin and Kohn [2008] showed that it suffices to use a walled structure (similar to the structure on the right in Figure 4, but with walls in many directions, not just two, and with the wall thickness depending on orientation).

As observed by Avellaneda [1987b], the implications of course also apply to 2-dimensional elasticity. Define

$$
W_{f}^{0}\left(\boldsymbol{\sigma}_{1}^{0}, \boldsymbol{\sigma}_{2}^{0}, \boldsymbol{\sigma}_{3}^{0}\right)=\min _{\boldsymbol{C}_{*} \in G U_{f}} \sum_{j=1}^{3} \boldsymbol{\sigma}_{j}^{0}: \boldsymbol{C}_{*}^{-1} \boldsymbol{\sigma}_{j}^{0}
$$

and

$$
W_{f}^{3}\left(\boldsymbol{\epsilon}_{1}^{0}, \boldsymbol{\epsilon}_{2}^{0}, \boldsymbol{\epsilon}_{3}^{0}\right)=\min _{\boldsymbol{C}_{*} \in G U_{f}} \sum_{i=1}^{3} \boldsymbol{\epsilon}_{i}^{0}: \boldsymbol{C}_{*} \boldsymbol{\epsilon}_{i}^{0} .
$$

Then there is an Avellaneda material with effective tensor $\boldsymbol{C}_{*}=\boldsymbol{C}_{f}^{A}\left(\boldsymbol{\epsilon}_{1}^{0}, \boldsymbol{\epsilon}_{2}^{0}, \boldsymbol{\epsilon}_{3}^{0}\right)$ that attains the minimum in (2-22), and a complementary Avellaneda material with effective tensor $\boldsymbol{C}_{*}=\widetilde{\boldsymbol{C}}_{f}^{A}\left(\boldsymbol{\sigma}_{1}^{0}, \boldsymbol{\sigma}_{2}^{0}, \boldsymbol{\sigma}_{3}^{0}\right) \in G U_{f}$ that attains the minimum in (2-21). In 2-dimensional elasticity, sequentially layered laminates have elasticity tensors given by (2-15)-(2-17) when the tensor $\boldsymbol{C}_{1}$ is anisotropic. When the elasticity tensor $\boldsymbol{C}_{1}$ of phase 1 is isotropic, the sequentially layered laminates of rank $r$ have effective compliance tensors $S_{*}=\left(\boldsymbol{C}_{*}\right)^{-1}$ given by the Gibiansky-Cherkaev formula

$$
(1-f)\left(\boldsymbol{S}_{1}-\boldsymbol{S}_{*}\right)^{-1}=\left(\boldsymbol{S}_{1}-\boldsymbol{S}_{2}\right)^{-1}-f\left[\left(4 \kappa_{2}\right)^{-1}+\left(4 \mu_{2}\right)^{-1}\right] \boldsymbol{M}
$$


(see [Gibiansky and Cherkaev 1997b, equations (2.37) and (2.38)] and see also [Lurie et al. 1982], in which Lurie, Cherkaev, and Fedorov derived an equivalent, but less simple, formula), where $\boldsymbol{S}_{1}=\left(\boldsymbol{C}_{1}\right)^{-1}$ and $\boldsymbol{S}_{2}=\left(\boldsymbol{C}_{2}\right)^{-1}$ are the compliance tensors of the two phases, occupying volume fractions $f$ and $1-f$, respectively, and $\boldsymbol{M}$ has elements

$$
\{\boldsymbol{M}\}_{h i k \ell}=\sum_{j=1}^{r} c_{j} \boldsymbol{t}_{j_{h}} \boldsymbol{t}_{j_{i}} \boldsymbol{t}_{j_{k}} \boldsymbol{t}_{j_{\ell}},
$$

in which the $\boldsymbol{t}_{j}$ are unit vectors perpendicular to the directions of lamination (i.e., parallel to the layer boundaries), and the $c_{j}$ are any set of positive weights, summing to 1 , giving the proportions of phase 1 laminated in the various directions. The tensor $\boldsymbol{M}$ is clearly positive semidefinite and has the property that

$$
\{\boldsymbol{M}\}_{h k h k}=\{\boldsymbol{M}\}_{h h k k}=1 .
$$

Conversely, Avellaneda and Milton [1989] have shown that given a positive semidefinite fourth-order tensor $\boldsymbol{M}$ satisfying (2-25) there is a sequential layered laminate of rank $r \leq 3$ that corresponds to it, i.e., such that (2-23) holds for some choice of unit vectors $\boldsymbol{t}_{j}$ and weights $c_{j}$ (see also Theorem 2.2 of [Francfort et al. 1995]). Thus when $\boldsymbol{C}_{1}$ is isotropic, the computation of the complementary Avellaneda tensor $\widetilde{\boldsymbol{C}}_{f}^{A}\left(\boldsymbol{\sigma}_{1}^{0}, \boldsymbol{\sigma}_{2}^{0}, \boldsymbol{\sigma}_{3}^{0}\right)$ reduces to a minimization over positive semidefinite fourth-order tensors $\boldsymbol{M}$ satisfying (2-25). When $\boldsymbol{C}_{1}$ is anisotropic, by the same argument as in the 3-dimensional case, it suffices to consider sequential layered laminates of rank at most 6 .

We also remark that aside from hierarchical laminates there are many other structures that have a uniform field in one phase, sometimes only for certain applied fields. These include assemblages of confocal ellipses and ellipsoids [Milton 1980; 1981a; Grabovsky and Kohn 1995a], the periodic Vigdergauz geometries [Vigdergauz 1986; 1994; 1996; 1999; Grabovsky and Kohn 1995b], the Sigmund structures [2000], and the periodic E-inclusions of Liu, James, and Leo [Liu et al. 2007] (see also Section 23.9 of [Milton 2002]). Usually these attain the bounds when the measure $\mu(\xi)$ minimizing the sum of Willis bounds is not required to be a discrete measure. Allaire and Aubry [1999] have shown that sometimes the best microstructure necessarily has structure on multiple length scales (like sequentially layered laminates).

For single energies for anisotropic two-phase composites, the Hill bounds (2-1) imply

$$
\begin{gathered}
\boldsymbol{\epsilon}_{0}:\left[f \boldsymbol{C}_{1}^{-1}+(1-f) \boldsymbol{C}_{2}^{-1}\right]^{-1} \boldsymbol{\epsilon}_{0} \leq \boldsymbol{\epsilon}_{0}: \boldsymbol{C}_{*} \boldsymbol{\epsilon}_{0} \leq \boldsymbol{\epsilon}_{0}:\left[f \boldsymbol{C}_{1}+(1-f) \boldsymbol{C}_{2}\right] \boldsymbol{\epsilon}_{0}, \\
\boldsymbol{\sigma}_{0}:\left[f \boldsymbol{C}_{1}+(1-f) \boldsymbol{C}_{2}\right]^{-1} \boldsymbol{\sigma}_{0} \leq \boldsymbol{\sigma}_{0}: \boldsymbol{C}_{*}^{-1} \boldsymbol{\sigma}_{0} \leq \boldsymbol{\sigma}_{0}:\left[f \boldsymbol{C}_{1}^{-1}+(1-f) \boldsymbol{C}_{2}^{-1}\right] \boldsymbol{\sigma}_{0} .
\end{gathered}
$$


Improved, and in fact sharp, upper and lower bounds on the elastic energy $\boldsymbol{\epsilon}_{0}: \boldsymbol{C}_{*} \boldsymbol{\epsilon}_{0}$ in terms of the given applied strain $\epsilon_{0}$ and sharp upper and lower bounds on the complementary elastic energy $\boldsymbol{\sigma}_{0}: \boldsymbol{C}_{*}^{-1} \boldsymbol{\sigma}_{0}$ in terms of the given applied stress $\boldsymbol{\sigma}_{0}$ were obtained for isotropic component materials by Gibiansky and Cherkaev [1997a], Kohn and Lipton [1988], and Allaire and Kohn [1993a; 1993b; 1994]. The paper of Gibiansky and Cherkaev [1997a] was for the fourth-order plate equation, but this can be mapped to the equivalent 2-dimensional elasticity problem considered by Allaire and Kohn [1993b]. Their lower bounds on $\boldsymbol{\sigma}^{0}: \boldsymbol{C}_{*}^{-1} \boldsymbol{\sigma}^{0}$ are equivalent to the bounds that for any tensor $\boldsymbol{C}_{*} \in G U_{f}$,

$$
\boldsymbol{\sigma}^{0}: \boldsymbol{C}_{*}^{-1} \boldsymbol{\sigma}^{0} \geq \boldsymbol{\sigma}^{0}:\left[\widetilde{\boldsymbol{C}}_{f}^{A}\left(\boldsymbol{\sigma}^{0}, 0,0\right)\right]^{-1} \boldsymbol{\sigma}^{0}
$$

and they provided an explicit formula for the right-hand side for any $2 \times 2$ symmetric matrix $\sigma^{0}$ representing the applied stress. This bound can be viewed in two ways: in the way originally interpreted, i.e., as a bound on the possible (elastic energy, average stress, volume fraction) triplets; or as a bound

$$
\boldsymbol{\sigma}^{0}: \boldsymbol{\epsilon}^{0} \geq \boldsymbol{\sigma}^{0}:\left[\widetilde{\boldsymbol{C}}_{f}^{A}\left(\boldsymbol{\sigma}^{0}, 0,0\right)\right]^{-1} \boldsymbol{\sigma}^{0}
$$

on the possible (average stress, average strain, volume fraction) triplets. Here $\epsilon^{0}=$ $\boldsymbol{C}_{*}^{-1} \boldsymbol{\sigma}^{0}$ is the strain associated with $\boldsymbol{\sigma}^{0}$. Significantly, Milton, Serkov, and Movchan [Milton et al. 2003] found that the inequality (2-28) completely characterizes the possible (average stress, average strain, volume fraction) triplets in the limit in which one phase becomes void, when the other phase is isotropic. Specifically, given any triplet $\left(\boldsymbol{\sigma}^{0}, \boldsymbol{\epsilon}^{0}, f\right)$ satisfying (2-28) as an inequality, they give a recipe for constructing a 2-dimensional microstructure with effective tensor $\boldsymbol{C}_{*}$ and having phase 1 occupy a volume fraction $f$ such that $\sigma^{0}=\boldsymbol{C}_{*} \boldsymbol{\epsilon}^{0}$.

For 3-dimensional composites explicit expressions for the optimal upper energy bound were found by Gibiansky and Cherkaev [1997b] and Allaire [1994] for the case of a two-phase composite where one of the phases is void or rigid [Gibiansky and Cherkaev 1997b]. Grabovsky [1996] obtained energy bounds for two-phase composites containing anisotropic phases, each with a constant orientation.

Another major advance was made by Milton and Cherkaev [1995], who showed that any desired positive definite fourth-order tensor which has the symmetries of an elasticity tensor could be realized as the effective elasticity tensor $\boldsymbol{C}_{*}$ of a composite of a sufficiently stiff isotropic material and a sufficiently compliant isotropic material. One key to this advance was the realization that certain structures called pentamode materials could be (arbitrarily) stiff to one applied stress $\sigma_{1}^{0}$ and yet have five mutually orthogonal strains $\boldsymbol{\epsilon}_{1}^{0}, \boldsymbol{\epsilon}_{2}^{0}, \boldsymbol{\epsilon}_{3}^{0}, \boldsymbol{\epsilon}_{4}^{0}, \boldsymbol{\epsilon}_{5}^{0}$, each orthogonal to $\boldsymbol{\sigma}_{1}^{0}$ as five (arbitrarily compliant) easy modes of deformations (hence the name pentamode). 


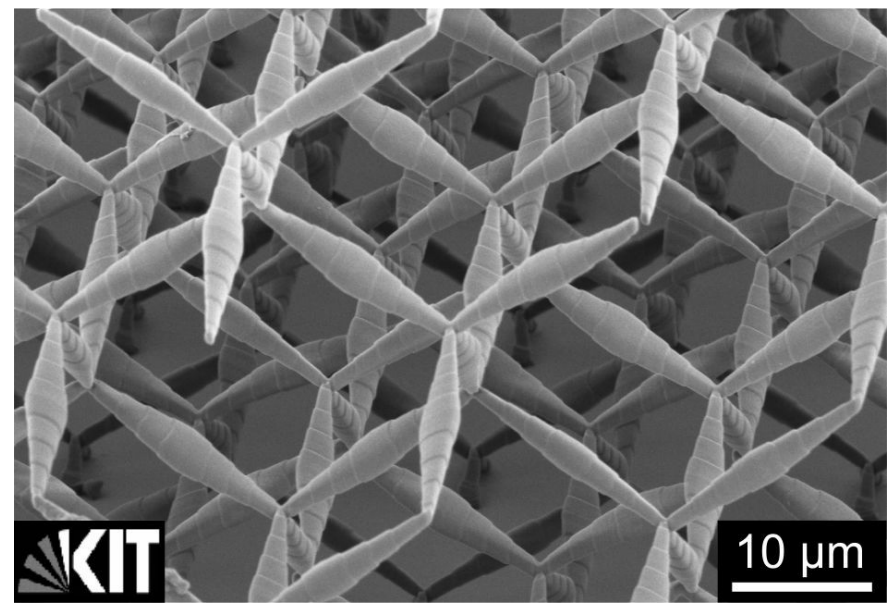

Figure 1. An electron micrograph of the pentamode structure created by Kadic, Bückmann, Stenger, Thiel and Wegener [Kadic et al. 2012] using a 3-dimensional lithography technique. (Used with the kind permission of Martin Wegener.)

For such a pentamode

$$
W_{f}^{5}\left(\boldsymbol{\sigma}_{1}^{0}, \boldsymbol{\epsilon}_{1}^{0}, \boldsymbol{\epsilon}_{2}^{0}, \boldsymbol{\epsilon}_{3}^{0}, \boldsymbol{\epsilon}_{4}^{0}, \boldsymbol{\epsilon}_{5}^{0}\right)=\min _{\boldsymbol{C}_{*} \in G U_{f}}\left[\left(\sum_{i=1}^{5} \boldsymbol{\epsilon}_{i}^{0}: \boldsymbol{C}_{*} \boldsymbol{\epsilon}_{i}^{0}\right)+\boldsymbol{\sigma}_{1}^{0}: \boldsymbol{C}_{*}^{-1} \boldsymbol{\sigma}_{1}^{0}\right]
$$

approaches zero as the constituent stiff isotropic material becomes increasingly stiff and the constituent compliant isotropic material becomes increasingly compliant. The lattice structure of a pentamode is similar to that of diamond with a stiff double cone structure replacing each carbon bond. This structure ensures that the tips of four double cone structures meet at each vertex. This is the essential feature: treating the double cone structures as struts, the tension in one determines uniquely the tension in the other three. This is simply the balance of forces. Thus the structure as a whole can essentially support only one stress. Pentamode structures were experimentally realized by Kadic, Bückmann, Stenger, Thiel and Wegener [Kadic et al. 2012] in an incredible feat of precision three-dimensional lithography. One of their electron micrographs of the structure is shown in Figure 1. Pentamode structures were also independently discovered in 1995 by Sigmund, although he did not find the complete span of pentamode structures needed here: one needs pentamodes that can support any chosen stress, not just a hydrostatic one. It is this aspect of pentamodes that makes them more interesting than, for example, a gel. Gels are examples of pentamodes as they are easy to shear, but difficult to compress under a hydrostatic loading $\sigma_{1}=\boldsymbol{I}$. By contrast the pentamodes of Milton and Cherkaev could be stiff to any desired stress $\sigma_{1}^{0}$ : this desired stress 
may be a mixture of shear and compression, and may have eigenvalues of mixed signs. A simple argument for seeing that these pentamodes can achieve any desired elasticity tensor was given in the foreword of the book edited by Phani and Hussein [2017]. To recapitulate that argument, one expresses the desired $\boldsymbol{C}_{*}$ in terms of its eigenvectors and eigenvalues,

$$
\boldsymbol{C}_{*}=\sum_{i=1}^{6} \lambda_{i} \boldsymbol{v}_{i} \otimes \boldsymbol{v}_{i} .
$$

The idea, roughly speaking, is to find six pentamode structures each supporting a stress represented by the vector $\boldsymbol{v}_{i}$ for $i=1,2, \ldots, 6$. The stiffness of the material and the necks of the junction regions at the vertices need to be adjusted so each pentamode structure has an effective elasticity tensor close to

$$
\boldsymbol{C}_{*}^{(i)}=\lambda_{i} \boldsymbol{v}_{i} \otimes \boldsymbol{v}_{i}
$$

Then one successively superimposes all these six pentamode structures, with their lattice structures being offset to avoid collisions. Additionally one may need to deform the structures appropriately to avoid these collisions as described in [Milton and Cherkaev 1995], and when one does this it is necessary to readjust the stiffness of the material in the structure to maintain the value of $\lambda_{i}$. Then the remaining void in the structure is replaced by an extremely compliant material. (Its presence is needed just for technical reasons, to ensure that the assumptions of homogenization theory are valid so that the elastic properties can be described by an effective tensor.) But it is so compliant that essentially the effective elasticity tensor is just a sum of the effective elasticity tensors of the six pentamodes, i.e., the elastic interaction between the six pentamodes is negligible. In this way we arrive at a material with (approximately) the desired elasticity tensor $\boldsymbol{C}_{*}$.

It is worth mentioning that with extremely high contrast materials the homogenized equations are not necessarily the usual linear elasticity equations, but can also include nonlocal terms. Nonlocal interactions can be obtained for example with an extremely stiff dumbbell-shaped inclusion with the balls arbitrarily distant. If the bar joining them is not only extremely stiff but also extremely thin, then it does not directly couple with the surrounding elastic material (except in the very near vicinity of the bar, where it is obviously deformed by it), but provides a nonlocal interaction between the balls. In fact, amazingly, Camar-Eddine and Seppecher [2003] have completely characterized all possible linear macroscopic behaviors of any high contrast composite: they showed that any energetically stable behavior can be obtained using materials with such dumbbell-shaped inclusions interacting at many length scales. Some interesting examples of high contrast materials with exotic effective behaviors have been given by Seppecher, Alibert, and dell'Isola [Seppecher et al. 2011]. 

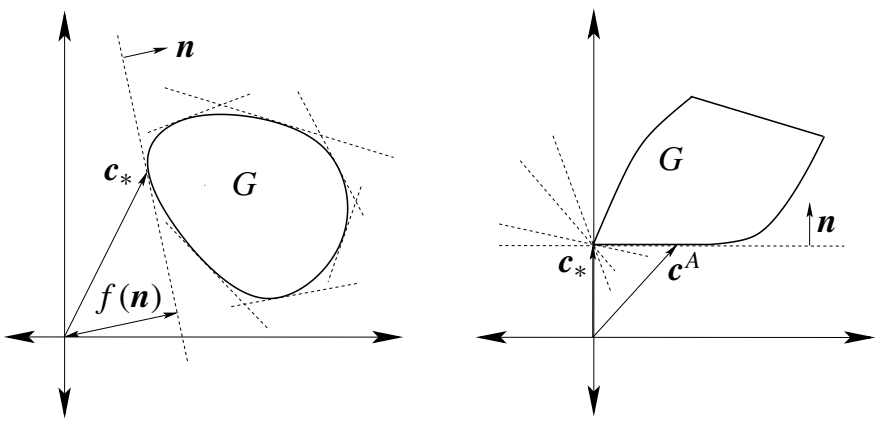

Figure 2. Left: A convex set is the envelope of its tangent planes. The positions of the two tangent planes with normal $\boldsymbol{n}$ are determined by the Legendre transform $f(\boldsymbol{n})$ and $f(-\boldsymbol{n})$ defined by (3-1). Specifically $f(\boldsymbol{n})$ and $f(-\boldsymbol{n})$ give the distances of the tangent planes from the origin. Right: An example highlighting an interesting case discussed in the text that helps give a geometrical interpretation of the results of the paper.

\section{Characterizing convex sets and $G$-closures for elasticity}

Let $G$ be a convex set of real $d$-dimensional vectors, meaning that if $c_{1}, c_{2} \in G$ then $\theta \boldsymbol{c}_{1}+(1-\theta) \boldsymbol{c}_{2} \in G$ for all $\theta \in[0,1]$. As shown in Figure 2 (left) for $d=2$ such a convex set can be completely characterized by its Legendre transform,

$$
f(\boldsymbol{n})=\min _{\boldsymbol{c} \in G} \boldsymbol{n} \cdot \boldsymbol{c} .
$$

Clearly this function satisfies the homogeneity property that

$$
f(\lambda \boldsymbol{n})=\lambda f(\boldsymbol{n}) \text { for all } \lambda>0,
$$

and consequently it suffices to know $f(\boldsymbol{n})$ for all unit vectors $\boldsymbol{n}$ to recover the function $f(\boldsymbol{n})$ for any vector $\boldsymbol{n}$. The values of $f(\boldsymbol{n})$ and $f(-\boldsymbol{n})$ give the positions of the two planes with normals $\pm \boldsymbol{n}$ that are tangent to $G$ : specifically $|f(\boldsymbol{n})|$ and $|f(-\boldsymbol{n})|$ give the distances from these tangent planes to the origin. By varying $\boldsymbol{n}$ and taking the intersection of the regions between the planes one recovers $G$ : the set $G$ is the envelope of its tangent planes as illustrated in Figure 2 (left). Thus the Legendre transform function $f(\boldsymbol{n})$ with $|\boldsymbol{n}|=1$ completely characterizes $G$.

The example of Figure 2 (right) is also illuminating for the purposes of this paper. Let $\boldsymbol{n}$ and $\boldsymbol{m}$ be the vectors

$$
\boldsymbol{n}=\left(\begin{array}{l}
0 \\
1
\end{array}\right), \quad \boldsymbol{m}=\left(\begin{array}{l}
1 \\
0
\end{array}\right)
$$

and consider $f(\boldsymbol{n}+\alpha \boldsymbol{m})$ for $\alpha \geq 0$ in the context of this example. (Of course $\boldsymbol{n}+\alpha \boldsymbol{m}$ is only a unit vector when $\alpha=0$.) As the boundary of $G$ contains a flat section 
orthogonal to $\boldsymbol{n}$, the vector $\boldsymbol{c}$ which attains the minimum in (3-1) is not unique. In the diagram both $\boldsymbol{c}^{A}$ and $\boldsymbol{c}_{*}$ are minimizers. However, for an infinitesimal value of $\alpha>0, \boldsymbol{c}_{*}$ is selected as the unique minimizer and remains the minimizer no matter how large $\alpha>0$ becomes. Furthermore, since $\boldsymbol{c}_{*}$ is orthogonal to $\boldsymbol{m}$ the value of $f(\boldsymbol{n}+\alpha \boldsymbol{m})$ remains constant for all $\alpha \geq 0$.

If $G$ is a convex set of, say, real $d \times d$ matrices it can be similarly characterized by its Legendre transform,

$$
f(\boldsymbol{N})=\min _{\boldsymbol{C} \in G}(\boldsymbol{N}, \boldsymbol{C}),
$$

defined for all $d \times d$ matrices $N$, where $(\boldsymbol{N}, \boldsymbol{C})$ is an inner product on the space of matrices which we may take to be

$$
(\boldsymbol{N}, \boldsymbol{C})=N_{i j} C_{i j} \equiv \boldsymbol{N}: \boldsymbol{C},
$$

where we have adopted the Einstein summation convention that sums over repeated indices are assumed, and the double dot ":" denotes a double contraction of indices. This is exactly equivalent to (3-1) if we think of the matrix $\boldsymbol{C}$ being represented by the vector $c$ of its matrix elements. Note that if $G$ only contains symmetric matrices, then it suffices to take $N$ as a symmetric matrix since $(\boldsymbol{A}, \boldsymbol{C})=0$ if $C$ is symmetric and $A$ is antisymmetric.

Similarly, if $G$ is a convex set of fourth-order elasticity tensors $C$ satisfying the usual symmetries

$$
C_{i j k \ell}=C_{j i k \ell}=C_{k \ell i j},
$$

then it can be characterized by the Legendre transform (3-4) with an inner product

$$
(\boldsymbol{N}, \boldsymbol{C})=N_{i j k \ell} C_{i j k \ell},
$$

and again it suffices to assume $\boldsymbol{N}$ has the same symmetries as $\boldsymbol{C}$, i.e., those in (3-6).

However, $G$-closures (i.e., sets of all possible effective tensors) are not generally convex sets. Nevertheless, they do have some convexity properties as a consequence of their stability under lamination. In the case of the set $G U_{f}$ where $U=\left\{\boldsymbol{C}_{1}, \delta \boldsymbol{C}_{2}\right\}$, we can take two materials with effective tensors $\boldsymbol{C}_{1}^{*}, \boldsymbol{C}_{2}^{*} \in G U_{f}$ and laminate them together in a direction $\boldsymbol{n}$ (representing the vector perpendicular to the layers) in proportions $\theta$ and $1-\theta$ to obtain an effective tensor $\boldsymbol{C}_{*}(\boldsymbol{n}, \theta)$ which necessarily lies in the set $G U_{f}$ for all $\theta \in[0,1]$. While $\boldsymbol{C}_{*}(\boldsymbol{n}, \theta)$ is not a linear average of $\boldsymbol{C}_{1}^{*}$ and $\boldsymbol{C}_{2}^{*}$, there exist fractional linear transformations $T_{\boldsymbol{n}}$ of fourth-order tensors such that lamination in direction $\boldsymbol{n}$ reduces to a linear average [Backus 1962; Milton 1990] (see also [Tartar 1979]):

$$
T_{\boldsymbol{n}}\left(\boldsymbol{C}_{*}(\boldsymbol{n}, \theta)\right)=\theta T_{\boldsymbol{n}}\left(\boldsymbol{C}_{1}^{*}\right)+(1-\theta) T_{\boldsymbol{n}}\left(\boldsymbol{C}_{2}^{*}\right) \text { for all } \theta \in[0,1] .
$$

Thus $T_{n}\left(G U_{f}\right)$ must be a convex set of fourth-order tensors. In the particular case where a set of effective tensors has no interior, i.e., is constrained to lie on a manifold of dimension $m$ smaller than the dimension of the space of fourth-order tensors 
satisfying the symmetries of elasticity tensors (i.e., $m<21$ for 3-dimensional composites and $m<6$ for 2-dimensional composites), then as recognized by Grabovsky [1998] (see also [Grabovsky and Sage 1998]) $T_{\boldsymbol{n}}$ must map this manifold to a subset of a hyperplane of dimension $m$ for any value of $\boldsymbol{n}$. This places rather severe constraints on the form of such manifolds. Identifying such manifolds is important as they represent exact relations satisfied by effective tensors, no matter what the geometry of the composite happens to be. Thus these constraints provide necessary conditions for an exact relation. Later, sufficient conditions for an exact relation to hold were obtained [Grabovsky et al. 2000].

Unfortunately, the use of Legendre transforms of the convex set $T_{n}\left(G U_{f}\right)$ is not useful to us as we are unaware of any direct variational principles for $T_{\boldsymbol{n}}\left(\boldsymbol{C}_{*}\right)$. An alternative approach was prompted by work of Cherkaev and Gibiansky [1992; 1993], who found that bounding sums of energies and complementary energies could lead to very useful bounds on $G$-closures. It was proved by Francfort and Milton [Francfort and Milton 1994; Milton 1994] that minimums over $\boldsymbol{C}_{*} \in G U_{f}$ of such sums of energies and complementary energies completely characterize $G U_{f}$ in much the same way that Legendre transforms characterize convex sets: the stability under lamination of $G U_{f}$ is what allows one to recover $G U_{f}$ from the values of these minimums (see also Chapter 30 in [Milton 2002]). Figure 3 captures the idea of this characterization.

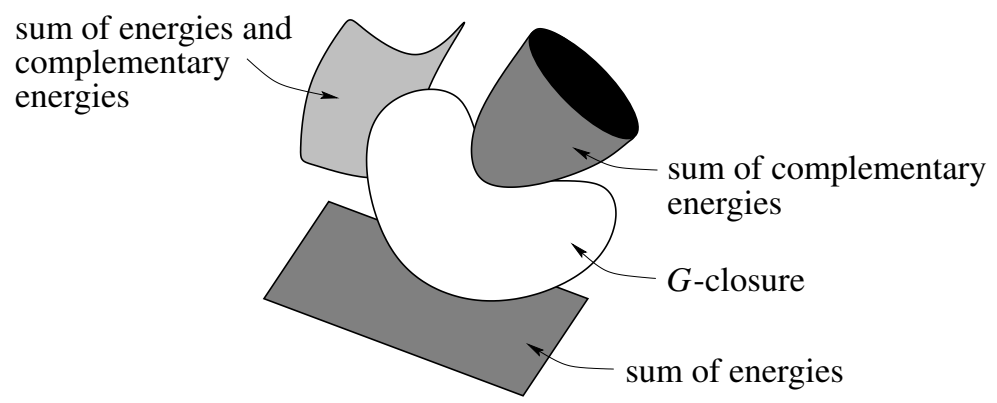

Figure 3. $G$-closures are characterized by minimums of sums of energies and complementary energies. The coordinates here represent the elements of the effective elasticity tensor $\boldsymbol{C}_{*}$. Then a plane represents a surface where a sum of energies is constant, and when this sum takes its minimum value the plane is tangent to the $G$-closure. The convexity properties of the $G$-closure guarantee that the surfaces corresponding to the minimums of sums of energies and complementary energies wrap around the $G$-closure and touch each point on its boundary. (Reproduction of Figure 30.1 in [Milton 2002].) 
Specifically, in the case of 3-dimensional elasticity, the set $G U_{f}$ is completely characterized if we know the seven "energy functions",

$$
\begin{aligned}
& W_{f}^{0}\left(\boldsymbol{\sigma}_{1}^{0}, \boldsymbol{\sigma}_{2}^{0}, \boldsymbol{\sigma}_{3}^{0}, \boldsymbol{\sigma}_{4}^{0}, \boldsymbol{\sigma}_{5}^{0}, \boldsymbol{\sigma}_{6}^{0}\right)=\min _{\boldsymbol{C}_{*} \in G U_{f}} \sum_{j=1}^{6} \boldsymbol{\sigma}_{j}^{0}: \boldsymbol{C}_{*}^{-1} \boldsymbol{\sigma}_{j}^{0}, \\
& W_{f}^{1}\left(\boldsymbol{\sigma}_{1}^{0}, \boldsymbol{\sigma}_{2}^{0}, \boldsymbol{\sigma}_{3}^{0}, \boldsymbol{\sigma}_{4}^{0}, \boldsymbol{\sigma}_{5}^{0}, \boldsymbol{\epsilon}_{1}^{0}\right)=\min _{\boldsymbol{C}_{*} \in G U_{f}}\left[\boldsymbol{\epsilon}_{1}^{0}: \boldsymbol{C}_{*} \boldsymbol{\epsilon}_{1}^{0}+\sum_{j=1}^{5} \boldsymbol{\sigma}_{j}^{0}: \boldsymbol{C}_{*}^{-1} \boldsymbol{\sigma}_{j}^{0}\right], \\
& W_{f}^{2}\left(\boldsymbol{\sigma}_{1}^{0}, \boldsymbol{\sigma}_{2}^{0}, \boldsymbol{\sigma}_{3}^{0}, \boldsymbol{\sigma}_{4}^{0}, \boldsymbol{\epsilon}_{1}^{0}, \boldsymbol{\epsilon}_{2}^{0}\right)=\min _{\boldsymbol{C}_{*} \in G U_{f}}\left[\sum_{i=1}^{2} \boldsymbol{\epsilon}_{i}^{0}: \boldsymbol{C}_{*} \boldsymbol{\epsilon}_{i}^{0}+\sum_{j=1}^{4} \boldsymbol{\sigma}_{j}^{0}: \boldsymbol{C}_{*}^{-1} \boldsymbol{\sigma}_{j}^{0}\right], \\
& W_{f}^{3}\left(\boldsymbol{\sigma}_{1}^{0}, \boldsymbol{\sigma}_{2}^{0}, \boldsymbol{\sigma}_{3}^{0}, \boldsymbol{\epsilon}_{1}^{0}, \boldsymbol{\epsilon}_{2}^{0}, \boldsymbol{\epsilon}_{3}^{0}\right)=\min _{\boldsymbol{C}_{*} \in G U_{f}}\left[\sum_{i=1}^{3} \boldsymbol{\epsilon}_{i}^{0}: \boldsymbol{C}_{*} \boldsymbol{\epsilon}_{i}^{0}+\sum_{j=1}^{3} \boldsymbol{\sigma}_{j}^{0}: \boldsymbol{C}_{*}^{-1} \boldsymbol{\sigma}_{j}^{0}\right], \\
& W_{f}^{4}\left(\boldsymbol{\sigma}_{1}^{0}, \boldsymbol{\sigma}_{2}^{0}, \boldsymbol{\epsilon}_{1}^{0}, \boldsymbol{\epsilon}_{2}^{0}, \boldsymbol{\epsilon}_{3}^{0}, \boldsymbol{\epsilon}_{4}^{0}\right)=\min _{\boldsymbol{C}_{*} \in G U_{f}}\left[\sum_{i=1}^{4} \boldsymbol{\epsilon}_{i}^{0}: \boldsymbol{C}_{*} \boldsymbol{\epsilon}_{i}^{0}+\sum_{j=1}^{2} \boldsymbol{\sigma}_{j}^{0}: \boldsymbol{C}_{*}^{-1} \boldsymbol{\sigma}_{j}^{0}\right], \\
& W_{f}^{5}\left(\boldsymbol{\sigma}_{1}^{0}, \boldsymbol{\epsilon}_{1}^{0}, \boldsymbol{\epsilon}_{2}^{0}, \boldsymbol{\epsilon}_{3}^{0}, \boldsymbol{\epsilon}_{4}^{0}, \boldsymbol{\epsilon}_{5}^{0}\right)=\min _{\boldsymbol{C}_{*} \in G U_{f}}\left[\left(\sum_{i=1}^{5} \boldsymbol{\epsilon}_{i}^{0}: \boldsymbol{C}_{*} \boldsymbol{\epsilon}_{i}^{0}\right)+\boldsymbol{\sigma}_{1}^{0}: \boldsymbol{C}_{*}^{-1} \boldsymbol{\sigma}_{1}^{0}\right], \\
& W_{f}^{6}\left(\boldsymbol{\epsilon}_{1}^{0}, \boldsymbol{\epsilon}_{2}^{0}, \boldsymbol{\epsilon}_{3}^{0}, \boldsymbol{\epsilon}_{4}^{0}, \boldsymbol{\epsilon}_{5}^{0}, \boldsymbol{\epsilon}_{6}^{0}\right)=\min _{\boldsymbol{C}_{*} \in G U_{f}} \sum_{i=1}^{6} \boldsymbol{\epsilon}_{i}^{0}: \boldsymbol{C}_{*} \boldsymbol{\epsilon}_{i}^{0} .
\end{aligned}
$$

In fact, it suffices [Milton and Cherkaev 1995] to know these functions for sets of applied strains $\boldsymbol{\epsilon}_{i}^{0}$ and applied stresses $\boldsymbol{\sigma}_{j}^{0}$ that are mutually orthogonal:

$$
\begin{aligned}
\left(\boldsymbol{\epsilon}_{i}^{0}, \boldsymbol{\sigma}_{j}^{0}\right)=0, \quad\left(\boldsymbol{\epsilon}_{i}^{0}, \boldsymbol{\epsilon}_{k}^{0}\right)=0, \quad\left(\boldsymbol{\sigma}_{j}^{0}, \boldsymbol{\sigma}_{\ell}^{0}\right)=0, & \\
& \text { for all } i, j, k, \ell \text { with } i \neq j, i \neq k, j \neq \ell .
\end{aligned}
$$

Each of these terms in the minimums has a physical significance. For example, in the expression for $W_{f}^{2}$,

$$
\sum_{i=1}^{2} \boldsymbol{\epsilon}_{i}^{0}: \boldsymbol{C}_{*} \boldsymbol{\epsilon}_{i}^{0}+\sum_{j=1}^{4} \boldsymbol{\sigma}_{j}^{0}: \boldsymbol{C}_{*}^{-1} \boldsymbol{\sigma}_{j}^{0}
$$

has the physical interpretation of being the sum of energies per unit volume stored in the composite with effective elasticity tensor $\boldsymbol{C}_{*}$ when successively subjected to the two applied strains $\epsilon_{1}^{0}$ and $\epsilon_{2}^{0}$ and then to the four applied stresses $\sigma_{1}^{0}, \sigma_{2}^{0}, \sigma_{3}^{0}$ and $\boldsymbol{\sigma}_{4}^{0}$. To distinguish the terms $\boldsymbol{\epsilon}_{i}^{0}: \boldsymbol{C}_{*} \boldsymbol{\epsilon}_{i}^{0}$ and $\boldsymbol{\sigma}_{j}^{0}: \boldsymbol{C}_{*}^{-1} \boldsymbol{\sigma}_{j}^{0}$, the first is called an energy (it is really an energy per unit volume associated with the applied strain $\epsilon_{i}^{0}$ ) 
and the second is called a complementary energy, although it too physically represents an energy per unit volume associated with the applied stress $\boldsymbol{\sigma}_{j}^{0}$. Note that the quantity (3-11) can be equivalently written as

$$
\left(C_{*}, N\right)+\left(C_{*}^{-1}, N^{\prime}\right),
$$

where

$$
\boldsymbol{N}=\sum_{i=1}^{2} \boldsymbol{\epsilon}_{i}^{0} \otimes \boldsymbol{\epsilon}_{i}^{0}, \quad \boldsymbol{N}^{\prime}=\sum_{j=1}^{4} \boldsymbol{\sigma}_{j}^{0} \otimes \boldsymbol{\sigma}_{j}^{0},
$$

in which for any $d \times d$ symmetric matrix $\boldsymbol{A}$, the tensor $\boldsymbol{A} \otimes \boldsymbol{A}$ is defined to be the fourth-order tensor with elements

$$
\{\boldsymbol{A} \otimes \boldsymbol{A}\}_{i j k \ell}=\{\boldsymbol{A}\}_{i j}\{\boldsymbol{A}\}_{k \ell} .
$$

If we decompose the positive semidefinite tensors $N$ and $N^{\prime}$ into their spectral decompositions

$$
\boldsymbol{N}=\sum_{i=1}^{2} \lambda_{i} \boldsymbol{v}_{i} \otimes \boldsymbol{v}_{i}, \quad \boldsymbol{N}^{\prime}=\sum_{j=1}^{4} \lambda_{j}^{\prime} \boldsymbol{v}_{j}^{\prime} \otimes \boldsymbol{v}_{j}^{\prime},
$$

with eigenmatrices $\boldsymbol{v}_{i}$ and $\boldsymbol{v}_{j}^{\prime}$ and corresponding nonnegative eigenvalues $\lambda_{i}$ and $\lambda_{j}^{\prime}$, then, with the orthogonality constraints (3-10), we can make the identifications

$$
\boldsymbol{\epsilon}_{i}^{0}=\sqrt{\lambda_{i}} \boldsymbol{v}_{i}, \quad \boldsymbol{\sigma}_{j}^{0}=\sqrt{\lambda_{j}^{\prime}} \boldsymbol{v}_{j} .
$$

Note that due to the orthogonality conditions (3-10) the fourth-order tensors $N$ and $N^{\prime}$ have the property that the product $N N^{\prime}$ is zero. Here the product of two fourth-order tensors $\boldsymbol{C}$ and $\boldsymbol{C}^{\prime}$ is given by

$$
\left\{\boldsymbol{C} \boldsymbol{C}^{\prime}\right\}_{i j k \ell}=\{\boldsymbol{C}\}_{i j m n}\left\{\boldsymbol{C}^{\prime}\right\}_{m n k \ell} .
$$

Thus in the same way that convex sets are the envelope of planes, the $G$-closure $G U_{f}$ is the envelope of special surfaces parametrized by positive semidefinite fourth-order tensors $\boldsymbol{N}$ and $\boldsymbol{N}^{\prime}$ satisfying the symmetries of elasticity tensors, and having zero product $N N^{\prime}=N^{\prime} N=0$ (i.e., the range of $N^{\prime}$ is in the null space of $N$, and conversely the range of $N$ is in the null space of $N^{\prime}$ ). These special surfaces consist of all positive definite fourth-order tensors $\boldsymbol{C}$ satisfying

$$
(C, N)+\left(C^{-1}, N^{\prime}\right)=c,
$$

where $c$ is a positive real constant. In the case $N^{\prime}=0$ this does represent a hyperplane, but its orientation is restricted by the fact that the outward normal to the surface $N$ is restricted to be a positive definite fourth-order tensor (by outward normal we mean the normal pointing away from the origin). Knowledge of the seven 
functions $W_{f}^{i}$ given by (3-9) is clearly equivalent to knowledge of the function

$$
W_{f}\left(\boldsymbol{N}, \boldsymbol{N}^{\prime}\right)=\min _{\boldsymbol{C}_{*} \in G U_{f}}\left(C_{*}, \boldsymbol{N}\right)+\left(\boldsymbol{C}_{*}^{-1}, \boldsymbol{N}^{\prime}\right)
$$

for all positive semidefinite fourth-order tensors $N$ and $N^{\prime}$ satisfying the symmetries of elasticity tensors and having $N N^{\prime}=0$. The formula for recovering $G U_{f}$ from $W_{f}\left(N, N^{\prime}\right)$ is then

$$
\bigcap_{\substack{\boldsymbol{N}, \boldsymbol{N}^{\prime} \geq 0 \\ \boldsymbol{N} \boldsymbol{N}^{\prime}=0}}\left\{\boldsymbol{C}:(\boldsymbol{C}, \boldsymbol{N})+\left(\boldsymbol{C}^{-1}, \boldsymbol{N}^{\prime}\right) \geq W_{f}\left(\boldsymbol{N}, \boldsymbol{N}^{\prime}\right)\right\}=G U_{f} .
$$

More generally if we replace $G U_{f}$ in (3-19) by another set $G$ of positive definite matrices, and if the left-hand side of (3-20) is again $G$, then we may say $G$ is "W-convex".

An explicit definition of $\mathrm{W}$-convexity is as follows: a set $G$ of positive definite symmetric matrices is said to be strictly $\mathrm{W}$-convex if $G$ is simply connected and if for every pair of positive semidefinite symmetric matrices $N$ and $N^{\prime}$, not both zero, the minimum in

$$
\min _{\boldsymbol{C} \in G}(C, N)+\left(C^{-1}, N^{\prime}\right)
$$

is uniquely attained by only one $C \in G$. Geometrically, $G$ is strictly W-convex if for all positive semidefinite symmetric matrices $N$ and $N^{\prime}$, not both zero, the surface that consists of all positive definite matrices $\boldsymbol{C}$ satisfying

$$
(\boldsymbol{C}, \boldsymbol{N})+\left(\boldsymbol{C}^{-1}, \boldsymbol{N}^{\prime}\right)=k,
$$

where $k$ is chosen as the smallest value for which this surface touches $G$, has the property that it touches $G$ at only one point. A set $G$ is W-convex if it is a limit of strictly W-convex sets. If the set $G$ has a smooth boundary, then the condition for W-convexity can be expressed in terms of the curvature of the boundary of $G$ : when $G$ is a set of matrices, this curvature at each point on the surface of $G$ is a fourthorder tensor; when $G$ is a set of fourth-order elasticity tensors, this curvature is an eighth-order tensor. (See equation (3.51) in [Milton 1994], or equation (30.11) in [Milton 2002], for the explicit inequalities that the curvature must satisfy.)

The stability of $G U_{f}$ under lamination implies it is $W$-convex, but $W$-convexity probably does not imply stability under lamination, as stability under lamination depends on the underlying partial differential equations. Associated with any set $G$ of symmetric positive definite matrices $\boldsymbol{C}$ is its $W$-transform, defined as

$$
W\left(\boldsymbol{N}, \boldsymbol{N}^{\prime}\right)=\min _{\boldsymbol{C} \in G}(\boldsymbol{C}, \boldsymbol{N})+\left(\boldsymbol{C}^{-1}, \boldsymbol{N}^{\prime}\right),
$$

where $N$ and $N^{\prime}$ are symmetric positive semidefinite matrices satisfying $N N^{\prime}=0$, and the inner product of two symmetric matrices $\boldsymbol{A}$ and $\boldsymbol{B}$ can be taken as $(\boldsymbol{A}, \boldsymbol{B})=$ 
$\operatorname{Tr}(\boldsymbol{A B})$, where $\operatorname{Tr}$ denotes the trace (sum of diagonal elements) of a matrix. To see some of the properties of $W$-transforms it is helpful to extend the definition of the transform to allow for matrices $\boldsymbol{N}$ and $\boldsymbol{N}^{\prime}$ that have a nonzero product, $\boldsymbol{N} \boldsymbol{N}^{\prime} \neq 0$. The defining equation, (3-23), remains the same. Then consider a weighted average of $\left(N_{1}, N_{1}^{\prime}\right)$ and $\left(N_{2}, N_{2}^{\prime}\right)$, with weights $\theta$ and $1-\theta$, where the four matrices $N_{1}, N_{1}^{\prime}, N_{2}, N_{2}^{\prime}$ are positive semidefinite. Then for any $\theta \in(0,1)$, we have

$$
\begin{aligned}
W( & \left.\theta \boldsymbol{N}_{1}+(1-\theta) \boldsymbol{N}_{2}, \theta \boldsymbol{N}_{1}^{\prime}+(1-\theta) \boldsymbol{N}_{2}^{\prime}\right) \\
& =\min _{\boldsymbol{C} \in G}\left\{\theta\left[\left(\boldsymbol{C}, \boldsymbol{N}_{1}\right)+\left(\boldsymbol{C}^{-1}, \boldsymbol{N}_{1}^{\prime}\right)\right]+(1-\theta)\left[\left(\boldsymbol{C}, \boldsymbol{N}_{2}\right)+\left(\boldsymbol{C}^{-1}, \boldsymbol{N}_{2}^{\prime}\right)\right]\right\} \\
& \geq \theta\left\{\min _{\boldsymbol{C} \in G}\left(\boldsymbol{C}, \boldsymbol{N}_{1}\right)+\left(\boldsymbol{C}^{-1}, \boldsymbol{N}_{1}^{\prime}\right)\right\}+(1-\theta)\left\{\min _{\boldsymbol{C} \in G}\left(\boldsymbol{C}, \boldsymbol{N}_{2}\right)+\left(\boldsymbol{C}^{-1}, \boldsymbol{N}_{2}^{\prime}\right)\right\} \\
& \geq \theta W_{f}\left(\boldsymbol{N}_{1}, \boldsymbol{N}_{1}^{\prime}\right)+(1-\theta) W_{f}\left(\boldsymbol{N}_{2}, \boldsymbol{N}_{2}^{\prime}\right),
\end{aligned}
$$

which (by definition) implies $W\left(\boldsymbol{N}, \boldsymbol{N}^{\prime}\right)$ is a jointly concave function of $\boldsymbol{N}$ and $\boldsymbol{N}^{\prime}$. This concavity is a well-known property of Legendre transforms.

\section{Variational principles}

Upper bounds on the sums of energies and complementary energies can easily be obtained from classic energy minimization variational principles. For example, in the case of the sum (3-11), we have

$$
\begin{aligned}
\sum_{i=1}^{2} \boldsymbol{\epsilon}_{i}^{0}: \boldsymbol{C}_{*} \boldsymbol{\epsilon}_{i}^{0}+\sum_{j=1}^{4} \boldsymbol{\sigma}_{j}^{0}: \boldsymbol{C}_{*}^{-1} \boldsymbol{\sigma}_{j}^{0} \\
\quad=\min _{\underline{\boldsymbol{\epsilon}}_{1}, \underline{\underline{\epsilon}}_{2}, \underline{\underline{\sigma}}_{1}, \underline{\boldsymbol{\sigma}}_{2}, \underline{\underline{\sigma}}_{3}, \underline{\underline{\sigma}}_{4}}\left\langle\sum_{i=1}^{2} \underline{\boldsymbol{\epsilon}}_{i}(\boldsymbol{x}): \boldsymbol{C}(\boldsymbol{x}) \underline{\boldsymbol{\epsilon}}_{i}(\boldsymbol{x})+\sum_{j=1}^{4} \underline{\boldsymbol{\sigma}}_{j}(\boldsymbol{x}):[\boldsymbol{C}(\boldsymbol{x})]^{-1} \underline{\boldsymbol{\sigma}}_{j}(\boldsymbol{x})\right\rangle,
\end{aligned}
$$

where the minimum is over a set of two trial strain fields $\underline{\epsilon}_{1}(\boldsymbol{x})$ and $\underline{\epsilon}_{2}(\boldsymbol{x})$ and a set of four trial stress fields $\underline{\sigma}_{1}(\boldsymbol{x}), \underline{\sigma}_{2}(\boldsymbol{x}), \underline{\sigma}_{3}(\boldsymbol{x})$, and $\underline{\sigma}_{4}(\boldsymbol{x})$ that have the prescribed average values

$$
\left\langle\underline{\boldsymbol{\epsilon}}_{i}\right\rangle=\boldsymbol{\epsilon}_{i}^{0} \quad \text { for } i=1,2, \quad\left\langle\underline{\boldsymbol{\sigma}}_{j}\right\rangle=\boldsymbol{\sigma}_{j}^{0} \quad \text { for } j=1,2,3,4,
$$

and are subject to the differential constraints that

$$
\begin{gathered}
\underline{\boldsymbol{\epsilon}}_{i}(\boldsymbol{x})=\frac{1}{2}\left(\nabla \underline{\boldsymbol{u}}_{i}(\boldsymbol{x})+\left(\nabla \underline{\boldsymbol{u}}_{i}(\boldsymbol{x})\right)^{T}\right) \quad \text { for } i=1,2, \\
\nabla \cdot \underline{\boldsymbol{\sigma}}_{j}(\boldsymbol{x})=0 \quad \text { for } j=1,2,3,4,
\end{gathered}
$$

where $T$ denotes the transpose (reflecting the matrix about its diagonal) and $\underline{\boldsymbol{u}}_{i}(\boldsymbol{x})$ is the trial displacement field associated with the trial stress field $\underline{\epsilon}_{i}(\boldsymbol{x})$. The trial strain fields $\underline{\epsilon}_{i}(\boldsymbol{x})$ and the trial stress fields $\underline{\boldsymbol{\sigma}}_{j}(\boldsymbol{x})$ (but not the trial displacement fields) should be chosen to be periodic (if the composite is periodic), quasiperiodic (if the composite is quasiperiodic), or statistically homogeneous (if the composite 
is statistically homogeneous). It may be the case that the material has structure on widely separated length scales. Maybe it can be viewed as a mixture of two composites, one with effective tensor $\boldsymbol{C}_{*}^{1}$ and a second with effective tensor $\boldsymbol{C}_{*}^{2}$, so that at the mesoscale it has a geometry described by a characteristic function $\chi_{*}(\boldsymbol{x})$, where $\chi_{*}(\boldsymbol{x})$ is 1 in the composite with effective tensor $\boldsymbol{C}_{*}^{1}$ and 0 in the material with effective tensor $\boldsymbol{C}_{*}^{2}$. Naturally the length scale, or length scales, of variations in $\chi_{*}(\boldsymbol{x})$ should be much larger than the variations in the microstructure of the materials that have the effective tensors $\boldsymbol{C}_{*}^{1}$ and $\boldsymbol{C}_{*}^{2}$. Then we can treat the material having effective tensor as a composite of the materials $\boldsymbol{C}_{*}^{1}$ and $\boldsymbol{C}_{*}^{2}$ and we have the variational principle

$$
\begin{aligned}
\sum_{i=1}^{2} \boldsymbol{\epsilon}_{i}^{0}: \boldsymbol{C}_{*} \boldsymbol{\epsilon}_{i}^{0}+\sum_{j=1}^{4} \boldsymbol{\sigma}_{j}^{0}: \boldsymbol{C}_{*}^{-1} \boldsymbol{\sigma}_{j}^{0} \\
=\min _{\underline{\boldsymbol{\epsilon}}_{1}, \underline{\boldsymbol{\epsilon}}_{2}, \underline{\boldsymbol{\sigma}}_{1}, \underline{\boldsymbol{\sigma}}_{2}, \underline{\sigma}_{3}, \underline{\boldsymbol{\sigma}}_{4}}\left\langle\sum_{i=1}^{2} \underline{\boldsymbol{\epsilon}}_{i}(\boldsymbol{x}):\left[\chi_{*}(\boldsymbol{x}) \boldsymbol{C}_{*}^{1}+\left(1-\chi_{*}(\boldsymbol{x})\right) \boldsymbol{C}_{*}^{2}\right] \underline{\boldsymbol{\epsilon}}_{i}(\boldsymbol{x})\right. \\
\left.+\sum_{j=1}^{4} \underline{\boldsymbol{\sigma}}_{j}(\boldsymbol{x}):\left[\chi_{*}(\boldsymbol{x}) \boldsymbol{C}_{*}^{1}+\left(1-\chi_{*}(\boldsymbol{x})\right) \boldsymbol{C}_{*}^{2}\right]^{-1} \underline{\boldsymbol{\sigma}}_{j}(\boldsymbol{x})\right\rangle,
\end{aligned}
$$

where again the minimum is over fields subject to the appropriate average values and differential constraints. Particular choices of trial fields will then lead to an upper bound on this sum of energies and complementary energies. To bound the quantities on the right one may again use variational principles. When $\boldsymbol{x}$ is in the material $\boldsymbol{C}_{*}^{k}$ for $k=1$ or 2 , one has the variational principles

$$
\begin{aligned}
& \underline{\boldsymbol{\epsilon}}_{i}(\boldsymbol{x}): \boldsymbol{C}_{* \underline{\boldsymbol{\epsilon}}_{i}}^{k}(\boldsymbol{x})=\min _{\underline{\boldsymbol{\epsilon}}_{i}}\left\langle\underline{\underline{\boldsymbol{\epsilon}}}_{i}(\boldsymbol{x}, \boldsymbol{y}): \boldsymbol{C}^{k}(\boldsymbol{y}) \underline{\underline{\boldsymbol{\epsilon}}}_{i}(\boldsymbol{x}, \boldsymbol{y})\right\rangle_{\boldsymbol{y}}, \\
& \underline{\boldsymbol{\sigma}}_{j}(\boldsymbol{x}):\left[\boldsymbol{C}_{*}^{k}\right]^{-1} \underline{\boldsymbol{\sigma}}_{j}(\boldsymbol{x})=\min _{\underline{\underline{\sigma}}_{j}}\left\langle\underline{\underline{\boldsymbol{\sigma}}}_{j}(\boldsymbol{x}, \boldsymbol{y}):\left[\boldsymbol{C}^{k}(\boldsymbol{y})\right]^{-1} \underline{\underline{\boldsymbol{\sigma}}}_{j}(\boldsymbol{x}, \boldsymbol{y})\right\rangle_{\boldsymbol{y}},
\end{aligned}
$$

where $\langle\cdot\rangle_{\boldsymbol{y}}$ now denotes an average over the $\boldsymbol{y}$ variable ( $\boldsymbol{x}$ is the "slow variable" and $\boldsymbol{y}$ is the "fast variable") and

$$
\boldsymbol{C}^{k}(\boldsymbol{y})=\chi^{k}(\boldsymbol{y}) \boldsymbol{C}_{1}+\left(1-\chi^{k}(\boldsymbol{y})\right) \boldsymbol{C}_{2},
$$

in which $\chi^{k}(\boldsymbol{y})$ is the characteristic function representing the geometry associated with the effective tensor $\boldsymbol{C}_{*}^{k}$, taking a value 1 in the material with tensor $\boldsymbol{C}_{1}$ and 0 in the material with tensor $\boldsymbol{C}_{2}$. Here the trial fields have the prescribed average values

$$
\left\langle\underline{\underline{\epsilon}}_{i}(\boldsymbol{x}, \boldsymbol{y})\right\rangle_{\boldsymbol{y}}=\underline{\boldsymbol{\epsilon}}_{i}(\boldsymbol{x}) \text { for } i=1,2, \quad\left\langle\underline{\underline{\sigma}}_{j}(\boldsymbol{x}, \boldsymbol{y})\right\rangle_{\boldsymbol{y}}=\underline{\sigma}_{j}(\boldsymbol{x}) \text { for } j=1,2,3,4,
$$

and are subject to the differential constraints

$$
\begin{gathered}
\underline{\boldsymbol{\epsilon}}_{i}(\boldsymbol{x}, \boldsymbol{y})=\frac{1}{2}\left(\nabla_{y} \underline{\underline{\boldsymbol{u}}}_{i}(\boldsymbol{x}, \boldsymbol{y})+\left(\nabla \underline{\underline{\underline{u}}}_{i}(\boldsymbol{x}, \boldsymbol{y})\right)^{T}\right) \quad \text { for } i=1,2, \\
\nabla \cdot y \underline{\underline{\sigma}}_{j}(\boldsymbol{x}, \boldsymbol{y})=0 \quad \text { for } j=1,2,3,4,
\end{gathered}
$$


where $\nabla_{y}$ and $\nabla \cdot y$ are the gradient and divergence with respect to the $\boldsymbol{y}$ variables. We call the step of replacing the variational principle (4-1) by the variational principles (4-4) and (4-5) the "homogenization at intermediate scales step".

In this paper we will choose trial fields that satisfy the local orthogonality condition that

$$
\underline{\epsilon}_{i}(\boldsymbol{x}): \underline{\boldsymbol{\sigma}}_{j}(\boldsymbol{x})=0, \quad \text { for all } \boldsymbol{x} .
$$

Using the differential constraints satisfied by the trial fields, and integration by parts, one sees that the associated average fields are necessarily orthogonal too:

$$
\boldsymbol{\epsilon}_{i}^{0}: \boldsymbol{\sigma}_{j}^{0}=\left\langle\underline{\boldsymbol{\epsilon}}_{i}(\boldsymbol{x})\right\rangle:\left\langle\underline{\boldsymbol{\sigma}}_{j}(\boldsymbol{x})\right\rangle=\left\langle\underline{\boldsymbol{\epsilon}}_{i}(\boldsymbol{x}): \underline{\boldsymbol{\sigma}}_{j}(\boldsymbol{x})\right\rangle=0 .
$$

\section{Finding most of the energy functions}

Recall from Section 2 that an complementary Avellaneda material is a sequentially layered laminate material with phase 1 occupying a volume fraction $f$ and with effective tensor

$$
\widetilde{\boldsymbol{C}}_{f}^{A}\left(\boldsymbol{\sigma}_{1}^{0}, \boldsymbol{\sigma}_{2}^{0}, \boldsymbol{\sigma}_{3}^{0}, \boldsymbol{\sigma}_{4}^{0}, \boldsymbol{\sigma}_{5}^{0}, 0\right)
$$

that attains equality in (2-13). It is found by minimizing the right-hand side of (2-13) as $\boldsymbol{C}_{*}$ varies within the class of tensors given by (2-15)-(2-17) with $\boldsymbol{C}_{2}=0$, as the rank $r$, the positive weights $c_{j}$ which sum to 1 , and the unit vectors $\boldsymbol{n}_{i}$ are varied. Here some of the applied stresses $\sigma_{j}^{0}$ could be zero. Since the energy $\boldsymbol{\sigma}_{j}^{0}: \boldsymbol{C}_{*}^{-1} \boldsymbol{\sigma}_{j}^{0}$ associated with any applied stress $\boldsymbol{\sigma}_{j}^{0}$ is necessarily nonnegative, we obtain from (3-9) the bounds

$$
\begin{gathered}
\sum_{j=1}^{5} \boldsymbol{\sigma}_{j}^{0}:\left[\widetilde{\boldsymbol{C}}_{f}^{A}\left(\boldsymbol{\sigma}_{1}^{0}, \boldsymbol{\sigma}_{2}^{0}, \boldsymbol{\sigma}_{3}^{0}, \boldsymbol{\sigma}_{4}^{0}, \boldsymbol{\sigma}_{5}^{0}, 0\right)\right]^{-1} \boldsymbol{\sigma}_{j}^{0} \leq W_{f}^{1}\left(\boldsymbol{\sigma}_{1}^{0}, \boldsymbol{\sigma}_{2}^{0}, \boldsymbol{\sigma}_{3}^{0}, \boldsymbol{\sigma}_{4}^{0}, \boldsymbol{\sigma}_{5}^{0}, \boldsymbol{\epsilon}_{1}^{0}\right), \\
\sum_{j=1}^{4} \boldsymbol{\sigma}_{j}^{0}:\left[\widetilde{\boldsymbol{C}}_{f}^{A}\left(\boldsymbol{\sigma}_{1}^{0}, \boldsymbol{\sigma}_{2}^{0}, \boldsymbol{\sigma}_{3}^{0}, \boldsymbol{\sigma}_{4}^{0}, 0,0\right)\right]^{-1} \boldsymbol{\sigma}_{j}^{0} \leq W_{f}^{2}\left(\boldsymbol{\sigma}_{1}^{0}, \boldsymbol{\sigma}_{2}^{0}, \boldsymbol{\sigma}_{3}^{0}, \boldsymbol{\sigma}_{4}^{0}, \boldsymbol{\epsilon}_{1}^{0}, \boldsymbol{\epsilon}_{2}^{0}\right), \\
\sum_{j=1}^{3} \boldsymbol{\sigma}_{j}^{0}:\left[\widetilde{\boldsymbol{C}}_{f}^{A}\left(\boldsymbol{\sigma}_{1}^{0}, \boldsymbol{\sigma}_{2}^{0}, \boldsymbol{\sigma}_{3}^{0}, 0,0,0\right)\right]^{-1} \boldsymbol{\sigma}_{j}^{0} \leq W_{f}^{3}\left(\boldsymbol{\sigma}_{1}^{0}, \boldsymbol{\sigma}_{2}^{0}, \boldsymbol{\sigma}_{3}^{0}, \boldsymbol{\epsilon}_{1}^{0}, \boldsymbol{\epsilon}_{2}^{0}, \boldsymbol{\epsilon}_{3}^{0}\right), \\
\sum_{j=1}^{2} \boldsymbol{\sigma}_{j}^{0}:\left[\widetilde{\boldsymbol{C}}_{f}^{A}\left(\boldsymbol{\sigma}_{1}^{0}, \boldsymbol{\sigma}_{2}^{0}, 0,0,0,0\right)\right]^{-1} \boldsymbol{\sigma}_{j}^{0} \leq W_{f}^{4}\left(\boldsymbol{\sigma}_{1}^{0}, \boldsymbol{\sigma}_{2}^{0}, \boldsymbol{\epsilon}_{1}^{0}, \boldsymbol{\epsilon}_{2}^{0}, \boldsymbol{\epsilon}_{3}^{0}, \boldsymbol{\epsilon}_{4}^{0}\right), \\
\boldsymbol{\sigma}_{1}^{0}:\left[\widetilde{\boldsymbol{C}}_{f}^{A}\left(\boldsymbol{\sigma}_{1}^{0}, 0,0,0,0,0\right)\right]^{-1} \boldsymbol{\sigma}_{1}^{0} \leq W_{f}^{5}\left(\boldsymbol{\sigma}_{1}^{0}, \boldsymbol{\epsilon}_{1}^{0}, \boldsymbol{\epsilon}_{2}^{0}, \boldsymbol{\epsilon}_{3}^{0}, \boldsymbol{\epsilon}_{4}^{0}, \boldsymbol{\epsilon}_{5}^{0}\right), \\
0 \leq W_{f}^{6}\left(\boldsymbol{\epsilon}_{1}^{0}, \boldsymbol{\epsilon}_{2}^{0}, \boldsymbol{\epsilon}_{3}^{0}, \boldsymbol{\epsilon}_{4}^{0}, \boldsymbol{\epsilon}_{5}^{0}, \boldsymbol{\epsilon}_{6}^{0}\right) .
\end{gathered}
$$

The last inequality is clearly sharp, being attained when the composite consists of islands of phase 1 surrounded by a phase 2 (so that $\boldsymbol{C}_{*}$ approaches 0 as $\delta \rightarrow 0$ ). 
The objective of this paper is to show that many of the other inequalities are also sharp in the limit $\delta \rightarrow 0$, at least when the spaces spanned by the applied strains $\boldsymbol{\epsilon}_{j}^{0}$ for $j=1,2, \ldots, p$ satisfy certain properties. This space of applied strains $\mathcal{V}_{p}$, associated with $W_{f}^{p}$, has dimension $p$ and is spanned by $\epsilon_{1}^{0}, \epsilon_{2}^{0}, \ldots, \epsilon_{p}^{0}$.

The recipe for doing this is to simply insert into a relevant complementary Avellaneda material a microstructure occupying a thin walled region, such that the material can slip along the walls when the applied strain lies in appropriate spaces $\mathcal{V}_{p}$, yet which is such that the combination of Avellaneda material and walled material can support without slip any applied stress in the subspace orthogonal to $\mathcal{V}_{p}$. This will be possible only when $\mathcal{V}_{p}$ is spanned by symmetrized rank 1 matrices, taking the form

$$
\boldsymbol{\epsilon}^{(k)}=\frac{1}{2}\left(\boldsymbol{a}_{k} \boldsymbol{n}_{k}^{T}+\boldsymbol{n}_{k} \boldsymbol{a}_{k}^{T}\right), \quad \text { for } k=1, \ldots, p .
$$

The existence of such matrices $\boldsymbol{\epsilon}^{(k)}$ is proved in Section 7. The proof uses small perturbations of the applied stresses and strains. But, due to the continuity of the energy functions $W_{f}^{k}$ established in Section 9, the small perturbations do not modify the generic result. The vectors $\boldsymbol{n}_{k}$ determine the orientation of the walls in the structure. For each $\boldsymbol{n}_{k}$ there is a set of parallel walls perpendicular to $\boldsymbol{n}_{k}$ that allow slip given by the strain $\boldsymbol{\epsilon}^{(k)}$. We say slip but it should be recognized that $\boldsymbol{\epsilon}^{(k)}$ is not generally a pure shear, but rather a combination of dilation and shear, since it does not generally have zero trace.

To define the thin walled structure, introduce the periodic function $H_{c}(x)$ with period 1 which takes the value 1 if $x-[x] \leq c$, where $[x]$ is the greatest integer less than $x$, and $c \in[0,1]$ gives the thickness of each wall relative to the spacing between walls (which is unity). Then for the unit vectors $\boldsymbol{n}_{1}, \boldsymbol{n}_{2}, \ldots, \boldsymbol{n}_{p}$ appearing in (5-2), and for a small relative wall thickness $c=\epsilon$, define the characteristic functions

$$
\eta_{k}(\boldsymbol{x})=H_{\epsilon}\left(\boldsymbol{x} \cdot \boldsymbol{n}_{k}+k / p\right) .
$$

This characteristic function defines a series of parallel walls, as shown on the left in Figure 4, each perpendicular to the vector $\boldsymbol{n}_{j}$, where $\eta_{j}(\boldsymbol{x})=1$ in the wall material. The additional shift term $k / p$ in (5-3) ensures the walls associated with $k_{1}$ and $k_{2}$ do not intersect when it happens that $\boldsymbol{n}_{k_{1}}=\boldsymbol{n}_{k_{2}}$, at least when $\epsilon$ is small. Note that $\epsilon$ is a volume fraction, not a homogenization parameter. We will be taking the limit $\epsilon \rightarrow 0$ after taking the homogenization limit.

Now define the characteristic function

$$
\chi_{*}(\boldsymbol{x})=\prod_{k=1}^{p}\left(1-\eta_{k}(\boldsymbol{x})\right) .
$$

If $p \leq 3$, this is usually a periodic function of $\boldsymbol{x}$, an exception being if $p=3$ and there are no nonzero integers $z_{1}, z_{2}$, and $z_{3}$ such that $z_{1} \boldsymbol{n}_{1}+z_{2} \boldsymbol{n}_{2}+z_{3} \boldsymbol{n}_{3}=0$. More 

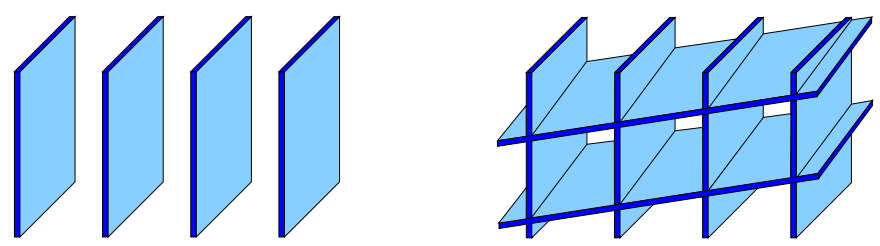

Figure 4. Example of walled structures. On the left we have a "rank 1" walled structure and on the right a "rank 2" walled structure. The generalization to walled structures of any rank is obvious, and precisely defined by the characteristic function (5-4) that is 0 in the walls, and 1 in the remaining material.

generally, $\chi_{*}(\boldsymbol{x})$ is a quasiperiodic function of $\boldsymbol{x}$. The walled structure is where $\chi_{*}(\boldsymbol{x})$ takes the value 0 . In the case $p=2$ the walled structure is illustrated on the right in Figure 4.

Recall that a $p$-mode material is a material for which there are $p$ independent strains to which the material is easily compliant, yet the material is much more resistant to any strain in the $(6-p)$-dimensional orthogonal subspace. In this sense the microstructure of Figure 1 is a pentamode material. We consider a subclass of multimode materials which can still support stresses in the limit $\delta \rightarrow 0$. We say a composite with effective tensor $\boldsymbol{C}_{*}$ built from the two materials $\boldsymbol{C}_{1}$ and $\boldsymbol{C}_{2}=\delta \boldsymbol{C}_{0}$ is easily compliant to a strain $\boldsymbol{\epsilon}_{i}^{0}$ if the elastic energy $\boldsymbol{\epsilon}_{i}^{0}: \boldsymbol{C}_{*} \boldsymbol{\epsilon}_{i}^{0}$ goes to zero as $\delta \rightarrow 0$, and supports a stress $\boldsymbol{\sigma}_{j}^{0}$ if the complementary energy $\boldsymbol{\sigma}_{j}^{0}: \boldsymbol{C}_{*}^{-1} \boldsymbol{\sigma}_{j}^{0}$ has a nonzero limit as $\delta \rightarrow 0$. We desire $p$-mode materials for which there are $p$ independent strains to which the material is easily compliant, yet for which the material supports any stress in the $(6-p)$-dimensional orthogonal subspace. The pentamode structure of Figure 1 needs to be modified as all its elastic moduli go to zero as $\delta \rightarrow 0$. The multimode structures we will introduce have structure on multiple length scales and it is important that one takes the limit of an infinite separation of length scales (so one can apply homogenization theory) before taking the limit $\delta \rightarrow 0$.

Inside the walled structure, where $\chi_{*}(\boldsymbol{x})=0$, we put a $p$-mode material with effective tensor $C_{*}^{2}=C_{*}\left(\mathcal{V}_{p}\right)$ that supports any applied stress $\sigma^{0}$ in the space orthogonal to $\mathcal{V}_{p}$ and which is easily compliant to any strain $\epsilon^{0}$ in the space $\mathcal{V}_{p}$. When we take the six matrices

$$
\boldsymbol{v}_{1}=\boldsymbol{\sigma}_{1}^{0} /\left|\boldsymbol{\sigma}_{1}^{0}\right|, \ldots, \boldsymbol{v}_{6-p}=\boldsymbol{\sigma}_{6-p}^{0} /\left|\boldsymbol{\sigma}_{6-p}^{0}\right|, \boldsymbol{v}_{7-p}=\boldsymbol{\epsilon}_{1}^{0} /\left|\boldsymbol{\epsilon}_{1}^{0}\right|, \ldots, \boldsymbol{v}_{6}=\boldsymbol{\epsilon}_{p}^{0} /\left|\boldsymbol{\epsilon}_{p}^{0}\right|
$$

as an orthonormal basis for the space of $6 \times 6$ matrices, we need to find a $p$-mode material for which the elasticity tensor $\boldsymbol{C}_{*}^{2}$ in this basis is such that

$$
\lim _{\delta \rightarrow 0} C_{*}^{2}=\left(\begin{array}{cc}
\boldsymbol{A} & 0 \\
0 & 0
\end{array}\right)
$$


where $A$ represents a (strictly) positive definite $(6-p) \times(6-p)$ matrix and the 0 on the diagonal represents the $p \times p$ zero matrix.

Outside the walled structure, where $\chi_{*}(\boldsymbol{x})=1$, we put the complementary Avellaneda material with effective elasticity tensor

$$
\boldsymbol{C}_{*}^{1}=\widetilde{\boldsymbol{C}}_{f}^{A}\left(\boldsymbol{\sigma}_{1}^{0}, \ldots, \boldsymbol{\sigma}_{6-p}^{0}, 0, \ldots, 0\right) .
$$

In a variational principle similar to (4-4) (i.e., treating the complementary Avellaneda material and the $p$-mode material both as homogeneous materials with effective tensors $\boldsymbol{C}_{*}^{1}$ and $\boldsymbol{C}_{*}^{2}$, respectively) we choose trial stress fields that are constant,

$$
\underline{\sigma}_{j}(\boldsymbol{x})=\boldsymbol{\sigma}_{j}^{0},
$$

thus trivially fulfilling the differential constraints, and trial strain fields of the form

$$
\underline{\boldsymbol{\epsilon}}_{i}(\boldsymbol{x})=\sum_{k=1}^{p} \boldsymbol{\epsilon}_{i, k} \eta_{k}(\boldsymbol{x}) / \epsilon,
$$

which are required to have the average values

$$
\boldsymbol{\epsilon}_{i}^{0}=\left\langle\underline{\boldsymbol{\epsilon}}_{i}\right\rangle=\sum_{k=1}^{p} \boldsymbol{\epsilon}_{i, k},
$$

and the matrices $\boldsymbol{\epsilon}_{i, k}$ have the form

$$
\boldsymbol{\epsilon}_{i, k}=a_{i, k} \boldsymbol{\epsilon}^{(k)},
$$

for some choice of constants $a_{i, k}$ which ensures they are symmetrized rank 1 matrices lying in the space $\mathcal{V}_{p}$ (so they cost very little energy), and which ensures that the $\epsilon_{i}^{0}$ given by (5-9) are orthogonal. This symmetrized rank 1 form ensures that $\underline{\epsilon}_{i}(\boldsymbol{x})$ derives from a displacement field. Specifically we have

$$
\underline{\boldsymbol{\epsilon}}_{i}(\boldsymbol{x})=\frac{1}{2}\left(\nabla \underline{\boldsymbol{u}}_{i}(\boldsymbol{x})+\left(\nabla \underline{\boldsymbol{u}}_{i}(\boldsymbol{x})\right)^{T}\right),
$$

with

$$
\underline{\boldsymbol{u}}_{i}(\boldsymbol{x})=\sum_{k=1}^{p} a_{i, k} \boldsymbol{a}_{k}\left\{\left(\boldsymbol{n}_{k} \cdot \boldsymbol{x}\right) \eta_{k}(\boldsymbol{x}) / \epsilon+\left(\left[\boldsymbol{n}_{k} \cdot \boldsymbol{x}\right]+1\right)\left(1-\eta_{k}(\boldsymbol{x})\right)\right\},
$$

where, as before, $\left[\boldsymbol{n}_{j} \cdot \boldsymbol{x}\right]$ is the greatest integer less than $\boldsymbol{n}_{j} \cdot \boldsymbol{x}$. One can easily check that this displacement field is continuous at the wall interfaces.

To find upper bounds on the energy associated with this trial strain field, first consider those parts of the walled structure that are outside of any junction regions, i.e., where for some $k$ we have $\eta_{k}(\boldsymbol{x})=1$, while $\eta_{s}(\boldsymbol{x})=0$ for all $s \neq k$. An upper bound for the volume fraction occupied by the region where $\eta_{k}(\boldsymbol{x})=1$ while $\eta_{s}(\boldsymbol{x})=0$ for all $s \neq k$ is of course $\epsilon$, as this represents the volume of the region 
where $\eta_{k}(\boldsymbol{x})=1$. The associated energy per unit volume of the trial strain field in those parts of the walled structure that are outside of any junction regions is bounded above by

$$
\sum_{k=1}^{p} \boldsymbol{\epsilon}_{i, k}: \boldsymbol{C}_{*}\left(\mathcal{V}_{p}\right) \boldsymbol{\epsilon}_{i, k} / \epsilon .
$$

We will see in Section 8 that with an appropriate choice of multimode material, $\boldsymbol{\epsilon}_{i, k}: \boldsymbol{C}_{*}\left(\mathcal{V}_{p}\right) \boldsymbol{\epsilon}_{i, k}$ is bounded above by a quantity proportional to $\delta$, essentially because all the strain is concentrated in phase 2 . So we require that the limits $\delta \rightarrow 0$ and $\epsilon \rightarrow 0$ be taken so that $\delta / \epsilon \rightarrow 0$ to ensure that the quantity (5-13) goes to zero in this limit.

Next, consider those junction regions where only two walls meet, i.e., where for some $k_{1}$ and $k_{2}>k_{1}, \boldsymbol{x}$ is such that $\eta_{k_{1}}(\boldsymbol{x})=\eta_{k_{2}}(\boldsymbol{x})=1$ while $\eta_{s}(\boldsymbol{x})=0$ for all $s$ not equal to $k_{1}$ or $k_{2}$. Provided $\boldsymbol{n}_{k_{1}} \neq \boldsymbol{n}_{k_{2}}$, an upper bound for the volume fraction occupied by each such junction region is $\epsilon^{2}$. Then the associated energy per unit volume of the trial strain field in these junction regions where only two walls meet is bounded above by

$$
\sum_{k_{1}=1}^{p} \sum_{k_{2}=k_{1}+1}^{p}\left(\boldsymbol{\epsilon}_{i, k_{1}}+\boldsymbol{\epsilon}_{i, k_{2}}\right): \boldsymbol{C}_{*}\left(\mathcal{V}_{p}\right)\left(\boldsymbol{\epsilon}_{i, k_{1}}+\boldsymbol{\epsilon}_{i, k_{2}}\right) .
$$

Thus, the powers of $\epsilon$ cancel and this energy density goes to zero if the multimode material is easily compliant to the strains $\boldsymbol{\epsilon}_{i, k_{1}}+\boldsymbol{\epsilon}_{i, k_{2}}$ for all $k_{1}$ and $k_{2}$ with $k_{2}>k_{1}$.

Finally, consider those junction regions where three or more walls meet, i.e., for some $k_{1}, k_{2}>k_{1}$, and $k_{3}>k_{2}, \boldsymbol{x}$ is such that $\eta_{k_{i}}(\boldsymbol{x})=1$ for $i=1,2,3$. For a given choice of $k_{1}, k_{2}>k_{1}$, and $k_{3}>k_{2}$ such that the three vectors $\boldsymbol{n}_{k_{1}}, \boldsymbol{n}_{k_{2}}$, and $\boldsymbol{n}_{k_{3}}$ are not coplanar, an upper bound for the volume fraction occupied by this region is $\epsilon^{3}$. In the case that the three vectors $\boldsymbol{n}_{k_{1}}, \boldsymbol{n}_{k_{2}}$, and $\boldsymbol{n}_{k_{3}}$ are coplanar, we can ensure that the volume fraction occupied by this region is $\epsilon^{3}$ or less by appropriately translating one or two wall structures, i.e., by replacing $\eta_{k_{m}}(\boldsymbol{x})$ with $\eta_{k_{m}}\left(\boldsymbol{x}+\alpha_{i} \boldsymbol{n}_{k_{m}}\right)$ for $m=2,3$, for an appropriate choice of $\alpha_{2}$ and $\alpha_{3}$ between 0 and 1. Since the energy density of the trial field in these regions scales as $\epsilon^{3} / \epsilon^{2}=\epsilon$, we can ignore this contribution in the limit $\epsilon \rightarrow 0$ as it goes to zero too.

From this analysis of the energy densities associated with the trial fields it follows that one does not necessarily need the pentamode, quadramode, trimode, bimode, and unimode materials as appropriate for the material inside the walled structure. Instead, by modifying the construction, it suffices to use only unimode and bimode materials. In the walled structure we now put unimode materials in those sections where for some $k$ we have $\eta_{k}(\boldsymbol{x})=1$ while $\eta_{k^{\prime}}(\boldsymbol{x})=0$ for all $k^{\prime} \neq k$. Each unimode material is easily compliant to the single strain $\epsilon^{(k)}$ appropriate to the wall under consideration. A prescription for constructing 3-dimensional unimode 
materials that are multiple rank laminates, and which are easily compliant under any desired single strain, is given in Section 5.1 of [Milton and Cherkaev 1995]. In each junction region of the walled structure where $\eta_{k_{1}}(\boldsymbol{x})=\eta_{k_{2}}(\boldsymbol{x})=1$ for some $k_{1} \neq k_{2}$ while $\eta_{k}(\boldsymbol{x})=0$ for all $k$ not equal to $k_{1}$ or $k_{2}$, we put a bimode material which is easily compliant to any strain in the subspace spanned by $\epsilon^{\left(k_{1}\right)}$ and $\boldsymbol{\epsilon}^{\left(k_{2}\right)}$ as appropriate to the junction region under consideration. At present we do not know of any recipe in three dimensions for constructing bimode materials that have any desired pair of strains as their easy modes of deformation, other than to superimpose four pentamode structures as described in Section 8. In the remaining junction regions of the walled structure (where three or more walls intersect) we put phase 1 . The contribution to the average energy of the fields in these regions vanishes as $\epsilon \rightarrow 0$ as discussed above.

By these constructions we effectively obtain materials with elasticity tensors $\boldsymbol{C}_{*}$ such that

$$
\lim _{\delta \rightarrow 0} \boldsymbol{C}_{*}=\left(\boldsymbol{I}-\Pi_{p}\right) \widetilde{\boldsymbol{C}}_{f}^{A}\left(\boldsymbol{I}-\Pi_{p}\right),
$$

where $\boldsymbol{I}$ is the fourth-order identity matrix, $\Pi_{p}$ is the fourth-order tensor that is the projection onto the space $\mathcal{V}_{p}, \boldsymbol{I}-\Pi_{p}$ is the projection onto the orthogonal complement of $\mathcal{V}_{p}$, and $\widetilde{\boldsymbol{C}}_{f}^{A}$ is the relevant complementary Avellaneda material. In the basis (5-5) $I-\Pi_{p}$ is represented by the $6 \times 6$ matrix that has the block form

$$
\boldsymbol{I}-\Pi_{p}=\left(\begin{array}{cc}
\boldsymbol{I}_{6-p} & 0 \\
0 & 0
\end{array}\right)
$$

where $\boldsymbol{I}_{6-p}$ represents the $(6-p) \times(6-p)$ identity matrix and the 0 on the diagonal represents the $p \times p$ zero matrix.

\section{Simplifications for 2-dimensional printed materials}

For 2-dimensional printed materials, or any 2-dimensional two-phase composite with one phase being void, the analysis simplifies as then the space of $2 \times 2$ symmetric matrices has dimension 3 , so there are only four energy functions to consider:

$$
\begin{aligned}
W_{f}^{0}\left(\boldsymbol{\sigma}_{1}^{0}, \boldsymbol{\sigma}_{2}^{0}, \boldsymbol{\sigma}_{3}^{0}\right) & =\min _{\boldsymbol{C}_{*} \in G U_{f}} \sum_{j=1}^{3} \boldsymbol{\sigma}_{j}^{0}: \boldsymbol{C}_{*}^{-1} \boldsymbol{\sigma}_{j}^{0}, \\
W_{f}^{1}\left(\boldsymbol{\sigma}_{1}^{0}, \boldsymbol{\sigma}_{2}^{0}, \boldsymbol{\epsilon}_{1}^{0}\right) & =\min _{\boldsymbol{C}_{*} \in G U_{f}}\left[\boldsymbol{\epsilon}_{1}^{0}: \boldsymbol{C}_{*} \boldsymbol{\epsilon}_{1}^{0}+\sum_{j=1}^{2} \boldsymbol{\sigma}_{j}^{0}: \boldsymbol{C}_{*}^{-1} \boldsymbol{\sigma}_{j}^{0}\right], \\
W_{f}^{2}\left(\boldsymbol{\sigma}_{1}^{0}, \boldsymbol{\epsilon}_{1}^{0}, \boldsymbol{\epsilon}_{2}^{0}\right) & =\min _{\boldsymbol{C}_{*} \in G U_{f}}\left[\left(\sum_{i=1}^{2} \boldsymbol{\epsilon}_{i}^{0}: \boldsymbol{C}_{*} \boldsymbol{\epsilon}_{i}^{0}\right)+\boldsymbol{\sigma}_{1}^{0}: \boldsymbol{C}_{*}^{-1} \boldsymbol{\sigma}_{1}^{0}\right], \\
W_{f}^{3}\left(\boldsymbol{\epsilon}_{1}^{0}, \boldsymbol{\epsilon}_{2}^{0}, \boldsymbol{\epsilon}_{3}^{0}\right) & =\min _{\boldsymbol{C}_{*} \in G U_{f}} \sum_{i=1}^{3} \boldsymbol{\epsilon}_{i}^{0}: \boldsymbol{C}_{*} \boldsymbol{\epsilon}_{i}^{0} .
\end{aligned}
$$


Again $W_{f}^{0}\left(\sigma_{1}^{0}, \sigma_{2}^{0}, \sigma_{3}^{0}\right)$ is attained for a "complementary Avellaneda material" consisting of a sequentially layered laminate geometry having an effective tensor $\boldsymbol{C}_{*}=$ $\widetilde{\boldsymbol{C}}_{f}^{A}\left(\boldsymbol{\sigma}_{1}^{0}, \boldsymbol{\sigma}_{2}^{0}, \boldsymbol{\sigma}_{3}^{0}\right) \in G U_{f}$, and we have the inequalities

$$
\begin{aligned}
\sum_{j=1}^{2} \boldsymbol{\sigma}_{j}^{0}:\left[\widetilde{\boldsymbol{C}}_{f}^{A}\left(\boldsymbol{\sigma}_{1}^{0}, \boldsymbol{\sigma}_{2}^{0}, 0\right)\right]^{-1} \boldsymbol{\sigma}_{j}^{0} & \leq W_{f}^{1}\left(\boldsymbol{\sigma}_{1}^{0}, \boldsymbol{\sigma}_{2}^{0}, \boldsymbol{\epsilon}_{1}^{0}\right), \\
\boldsymbol{\sigma}_{1}^{0}:\left[\widetilde{\boldsymbol{C}}_{f}^{A}\left(\boldsymbol{\sigma}_{1}^{0}, 0,0\right)\right]^{-1} \boldsymbol{\sigma}_{1}^{0} & \leq W_{f}^{2}\left(\boldsymbol{\sigma}_{1}^{0}, \boldsymbol{\epsilon}_{1}^{0}, \boldsymbol{\epsilon}_{2}^{0}\right), \\
0 & \leq W_{f}^{3}\left(\boldsymbol{\epsilon}_{1}^{0}, \boldsymbol{\epsilon}_{2}^{0}, \boldsymbol{\epsilon}_{3}^{0}\right),
\end{aligned}
$$

where, as before, the last inequality is sharp in the limit $\delta \rightarrow 0$ being attained when the material consists of islands of phase 1 surrounded by a phase 2 .

The recipe for showing that the bound (6-1) on $W_{f}^{1}\left(\boldsymbol{\sigma}_{1}^{0}, \boldsymbol{\sigma}_{2}^{0}, \boldsymbol{\epsilon}_{1}^{0}\right)$ is sharp for certain values of $\epsilon_{1}^{0}$ and that the bound (6-1) on $W_{f}^{2}\left(\boldsymbol{\sigma}_{1}^{0}, \boldsymbol{\epsilon}_{1}^{0}, \boldsymbol{\epsilon}_{2}^{0}\right)$ is sharp for certain values of $\epsilon_{1}^{0}$ and $\epsilon_{2}^{0}$ is almost exactly the same as in the 3-dimensional case: insert into the complementary Avellaneda material a thin walled structure of respectively unimode and bimode materials so that slips can occur along these walls, allowing with very little energetic cost the average strain $\epsilon_{1}^{0}$ in the case of $W_{f}^{1}$, or any strain in the space spanned by $\epsilon_{1}^{0}$ and $\epsilon_{2}^{0}$ in the case of $W_{f}^{2}$.

\section{The algebraic problem: characterizing those symmetric matrix pencils spanned by symmetrized rank 1 matrices}

We are interested in the following question: Given $k$ linearly independent symmetric $d \times d$ matrices $\boldsymbol{A}_{1}, \boldsymbol{A}_{2}, \ldots, \boldsymbol{A}_{k}$, find necessary and sufficient conditions such that there exist linearly independent matrices $\left\{\boldsymbol{B}_{i}\right\}_{i=1}^{k}$ spanned by the basis elements $\boldsymbol{A}_{i}$ so that each matrix $\boldsymbol{B}_{i}$ is a symmetrized rank 1 matrix, i.e., there exist vectors $\boldsymbol{a}_{i}$ and $\boldsymbol{b}_{i}$, with $\left|\boldsymbol{b}_{i}\right|=1$, such that

$$
\boldsymbol{B}_{i}=\frac{1}{2}\left(\boldsymbol{b}_{i} \boldsymbol{a}_{i}^{T}+\boldsymbol{a}_{i} \boldsymbol{b}_{i}^{T}\right) .
$$

It is assumed that $d=2$ or 3 and $1 \leq k \leq k_{d}$, where $k_{2}=2$ and $k_{3}=5$. Here we are working in the generic situation, i.e., we prove the algebraic result for a dense set of matrices. The continuity result of Section 9 will allow us to conclude for the whole set of matrices. Actually, the proof below also shows that the algebraic result holds for the complement of a zero measure set of matrices.

Theorem 7.1. The above problem is solvable if and only if the matrices $\boldsymbol{A}_{i}$ for $i=1, \ldots, k$ satisfy the following conditions:

$$
\operatorname{det}\left(\boldsymbol{A}_{1}\right) \leq 0, \quad \text { if } k=1, d=2,
$$

$A_{1}$ has two eigenvalues of opposite signs and one zero eigenvalue, or has two zero eigenvalues, if $k=1, d=3$. 
(ii) If $k=d=2$,

$$
\operatorname{det}\left(\boldsymbol{A}_{1}\right)<0
$$

or

$f(t)=\operatorname{det}\left(\boldsymbol{A}_{1}+t \boldsymbol{A}_{2}\right)$ is quadratic and has two distinct roots for $t$, or is linear in $t$ with a nonzero coefficient of $t$.

(iii) If $k=2$ and $d=3$, defining $\boldsymbol{A}(\eta, \mu)=\eta \boldsymbol{A}_{1}+\mu \boldsymbol{A}_{2}$, the numbers

$$
\operatorname{det}(\boldsymbol{A}(\eta, \mu)), \quad\{\boldsymbol{A}(\eta, \mu)\}_{11}\{\boldsymbol{A}(\eta, \mu)\}_{22}-\{\boldsymbol{A}(\eta, \mu)\}_{12}^{2}, \quad\{\boldsymbol{A}(\eta, \mu)\}_{11}
$$

are never simultaneously nonnegative for any choice of $\eta$ and $\mu$ not both zero (equivalently $\boldsymbol{A}(\eta, \mu)$ is never strictly positive definite for any values of $\eta$ and $\mu)$, and

$$
\begin{aligned}
\triangle=18 \operatorname{det}\left(\boldsymbol{A}_{1}\right) \operatorname{det}\left(\boldsymbol{A}_{2}\right) S_{1} S_{2}-4 S_{1}^{3} \operatorname{det}\left(\boldsymbol{A}_{2}\right) & +S_{1}^{2} S_{2}^{2}-4 S_{2}^{3} \operatorname{det}\left(\boldsymbol{A}_{1}\right) \\
& -27 \operatorname{det}\left(\boldsymbol{A}_{1}\right)^{2} \operatorname{det}\left(\boldsymbol{A}_{2}\right)^{2}>0,
\end{aligned}
$$

where $S_{i}=\sum_{j=1}^{3} s_{i j}$ for $i=1,2$ and $s_{i j}$ is the determinant of the matrix obtained by replacing the $j$-th row of $\boldsymbol{A}_{i}$ by the $j$-th row of $\boldsymbol{A}_{i+1}$, where by convention we have $\boldsymbol{A}_{3}=\boldsymbol{A}_{1}$ (equivalently $\boldsymbol{A}(\eta, \mu)$ has three distinct roots).

$$
\text { Always solvable if } k \geq 3, d=3 \text {. }
$$

Remark. In fact, the condition (7-2) and the last condition in (7-3), that $f(t)$ is linear in $t$, could be withdrawn since we are considering the generic case. They are inserted because we can treat them explicitly.

Proof. Case (i): $k=1, d=2$ or 3 . In this case $\boldsymbol{A}_{1}$ must be a multiple of $\boldsymbol{B}_{1}$ and hence must be a symmetrized rank 1 matrix. To see more clearly the condition for a matrix $\boldsymbol{B}$ to be a symmetrized rank 1 matrix, i.e., have the form $\boldsymbol{B}=\frac{1}{2}\left(\boldsymbol{b} \boldsymbol{a}^{T}+\boldsymbol{a} \boldsymbol{b}^{T}\right)$, let us, without loss of generality, choose our coordinates so that $\boldsymbol{b}=[1,0]^{T}$ when $d=2$ and $\boldsymbol{b}=[1,0,0]^{T}$ when $d=3$. Then $\boldsymbol{B}$ has the representation

$$
\boldsymbol{B}=\left(\begin{array}{cc}
a_{1} & \frac{1}{2} a_{2} \\
\frac{1}{2} a_{2} & 0
\end{array}\right) \text { when } d=2, \quad \boldsymbol{B}=\left(\begin{array}{ccc}
a_{1} & \frac{1}{2} a_{2} & \frac{1}{2} a_{3} \\
\frac{1}{2} a_{2} & 0 & 0 \\
\frac{1}{2} a_{3} & 0 & 0
\end{array}\right) \text { when } d=3 .
$$

These have eigenvalues

$$
\begin{aligned}
& \lambda=\frac{1}{2}\left(a_{1} \pm \sqrt{a_{1}^{2}+a_{2}^{2}}\right) \quad \text { when } d=2, \\
& \lambda=\frac{1}{2}\left(a_{1} \pm \sqrt{a_{1}^{2}+a_{2}^{2}+a_{3}^{2}}\right) \text { and } \lambda=0 \quad \text { when } d=3 .
\end{aligned}
$$

So, clearly $\boldsymbol{B}$ is a symmetrized rank 1 matrix in two dimensions if and only if $\operatorname{det}(\boldsymbol{B}) \leq 0$, and is a symmetrized rank 1 matrix in three dimensions if and only if 
it has two eigenvalues of opposite signs and one zero eigenvalue, or has two zero eigenvalues.

Case (ii): $k=2, d=2$. In this case there should be two distinct values of $t$ such that $\operatorname{det}\left(\boldsymbol{A}_{1}+t \boldsymbol{A}_{2}\right)<0$, which by continuity of this determinant as a function of $t$ is guaranteed if any of the conditions in (7-3) are met. Note that the case where $\operatorname{det}\left(\boldsymbol{A}_{1}+t \boldsymbol{A}_{2}\right)=0$ for all $t$ can be ruled out from consideration since this can only happen when $\boldsymbol{A}_{2}$ is proportional to $\boldsymbol{A}_{1}$, as can be easily seen by working in a basis where $\boldsymbol{A}_{2}$ is diagonal.

Case (iii): $k=2, d=3$. Consider the matrix pencil (over reals $\eta$ and $\mu$ ) $\boldsymbol{A}(\eta, \mu)=$ $\eta \boldsymbol{A}_{1}+\mu \boldsymbol{A}_{2}$. Assuming that $\operatorname{det} \boldsymbol{A}(\eta, \mu)$ is not zero for all $\eta$ and $\mu$, there are at least two matrices on the pencil which have nonzero determinant. Let us relabel them as $\boldsymbol{A}_{1}$ and $\boldsymbol{A}_{2}$. Then the equation $\operatorname{det}(\boldsymbol{A}(1, \mu))=0$ must have either two or three roots $\mu=z_{i}$ for $i=1,2$ or $i=1,2,3$, where the $z_{i}$ are obtained by changing the sign of the generalized eigenvalues. This gives Cardan's condition:

$$
\begin{aligned}
\triangle=18 \operatorname{det}\left(\boldsymbol{A}_{1}\right) \operatorname{det}\left(\boldsymbol{A}_{2}\right) S_{1} S_{2}-4 S_{1}^{3} \operatorname{det}\left(\boldsymbol{A}_{2}\right) & +S_{1}^{2} S_{2}^{2}-4 S_{2}^{3} \operatorname{det}\left(\boldsymbol{A}_{1}\right) \\
& -27 \operatorname{det}\left(\boldsymbol{A}_{1}\right)^{2} \operatorname{det}\left(\boldsymbol{A}_{2}\right)^{2} \geq 0 .
\end{aligned}
$$

Suppose that $\boldsymbol{A}_{1}+\mu \boldsymbol{A}_{2}$ contains a symmetric matrix with two zero eigenvalues (a rank 1 matrix) as $\mu$ is varied. Then by redefining $\boldsymbol{A}_{2}$ we can assume $\boldsymbol{A}_{2}$ is this matrix, now with zero determinant, and by using a basis where $\boldsymbol{A}_{2}$ is diagonal, we see that $\operatorname{det}\left(\boldsymbol{A}_{1}+\mu \boldsymbol{A}_{2}\right)$ depends linearly on $\mu$ and $\operatorname{det}\left(\boldsymbol{A}_{1}+\mu \boldsymbol{A}_{2}\right)$ can only have one root: (7-9) must be violated. So we can exclude this possibility: $\boldsymbol{A}_{1}+\mu \boldsymbol{A}_{2}$ has at most one zero eigenvalue for any value of $\mu$. Now consider the eigenvalues of $\boldsymbol{A}(\theta) \equiv \boldsymbol{A}(\cos \theta, \sin \theta)$ as $\theta$ is varied. As $\boldsymbol{A}(-\theta)=-\boldsymbol{A}(\theta)$ it suffices to consider the interval of $\theta$ between 0 and $\pi$. Some scenarios for the eigenvalue trajectories are plotted in Figure 5. At the values $\theta_{i}=\arctan ^{-1}\left(z_{i}\right)$ at least one of the eigenvalues must be zero, and the favorable situation is when there are two remaining eigenvalues of opposite signs or only one nonzero eigenvalue. Such angles $\theta_{i}$ are marked by the vertical dashed lines in the figure. The unfavorable situation is when there are two nonzero eigenvalues of the same sign, marked by the red vertical lines in Figure 5 (left). First suppose that $\boldsymbol{A}(\theta)$ is positive definite for some $\theta=\theta_{0}$. By refining $\theta$ as the old $\theta$ minus $\theta_{0}$, let us suppose $\boldsymbol{A}(0)$ is positive definite. Then the scenario is that in Figure 5 (left), or some variant of it in which eigenvalues cross, which is unfavorable. The only way to avoid this is for $\boldsymbol{A}(\theta)$ to have two zero eigenvalues at the smallest and largest values of $\theta \in[0, \pi]$ for which $\operatorname{det} \boldsymbol{A}(\theta)=0$, as in Figure 5 (middle), but we have ruled out the possibility that $\boldsymbol{A}(\theta)$ has two zero eigenvalues for any value of $\theta$. We are left with Figure 5 (right) as being the only possible suitable scenario. In conclusion, we require that the matrix $\boldsymbol{A}(\theta)$ not be positive semidefinite for any choice of $\theta$; i.e., the three quantities 

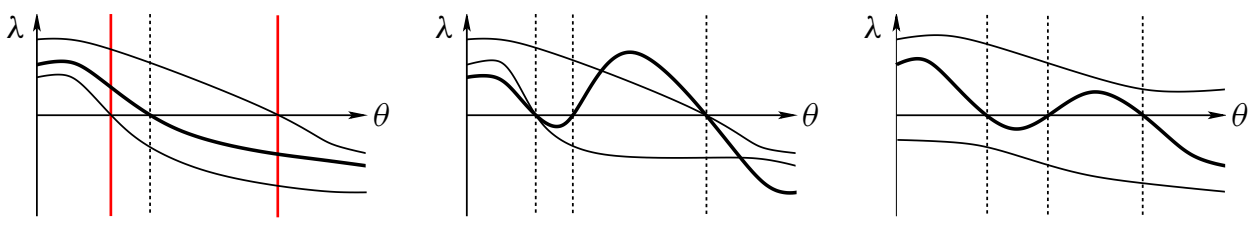

Figure 5. Some scenarios for the eigenvalues $\lambda$ of $\boldsymbol{A}(\theta)=$ $\cos \theta \boldsymbol{A}_{1}+\sin \theta \boldsymbol{A}_{2}$ as $\theta$ is varied.

$$
\operatorname{det}(\boldsymbol{A}(\eta, \mu)), \quad\{\boldsymbol{A}(\eta, \mu)\}_{11}\{\boldsymbol{A}(\eta, \mu)\}_{22}-\{\boldsymbol{A}(\eta, \mu)\}_{12}^{2}, \quad\{\boldsymbol{A}(\eta, \mu)\}_{11}
$$

are never simultaneously nonnegative for any choice of $\eta$ and $\mu$ not both zero. This condition could be made explicit by using the formula for the roots of a cubic to determine the generalized eigenvalues $-z_{i}$.

Case (iv): $k \geq 3, d=3$. The case $k=3$ is a straightforward consequence of Lemma 7.2 below.

It remains to consider $k \geq 4$ and $d=3$. By the previous step, in the space spanned by $\boldsymbol{A}_{1}, \boldsymbol{A}_{2}$, and $\boldsymbol{A}_{3}$ there are three matrices $\boldsymbol{A}_{1}^{\prime}, \boldsymbol{A}_{2}^{\prime}$, and $\boldsymbol{B}_{3}=\boldsymbol{A}_{3}+\eta_{3} \boldsymbol{A}_{1}^{\prime}+\mu_{3} \boldsymbol{A}_{2}^{\prime}$ that are linearly independent, symmetrized and of rank 1 . Then, again by the previous step, we can find linearly independent matrices $\boldsymbol{B}_{1}, \ldots, \boldsymbol{B}_{k}$ that have the form $\boldsymbol{B}_{1}=\boldsymbol{A}_{1}^{\prime}, \boldsymbol{B}_{2}=\boldsymbol{A}_{2}^{\prime}$, and $\boldsymbol{B}_{i}=\boldsymbol{A}_{i}+\eta_{i} \boldsymbol{A}_{1}^{\prime}+\mu_{i} \boldsymbol{A}_{2}^{\prime}$ for $3 \leq i \leq k$ and that are of rank 1 .

In the sequel we write

$$
\boldsymbol{a} \otimes \boldsymbol{b}:=\boldsymbol{a} \boldsymbol{b}^{T} \quad \text { and } \quad \boldsymbol{a} \odot \boldsymbol{b}:=\frac{1}{2}(\boldsymbol{a} \otimes \boldsymbol{b}+\boldsymbol{b} \otimes \boldsymbol{a}) \quad \text { for } \boldsymbol{a}, \boldsymbol{b} \in \mathbb{R}^{3} .
$$

Lemma 7.2. Let $\boldsymbol{A}, \boldsymbol{B}, \boldsymbol{C}$ be three symmetric matrices of $\mathbb{R}^{3 \times 3}$.

(i) Up to small perturbations of $\boldsymbol{A}, \boldsymbol{B}, \boldsymbol{C}$, there exist a basis $(\boldsymbol{x}, \boldsymbol{y}, \boldsymbol{z})$ of $\mathbb{R}^{3}$ and three vectors $\boldsymbol{a}, \boldsymbol{b}, \boldsymbol{c}$ of $\mathbb{R}^{3}$ satisfying

$$
\left\{\begin{array}{l}
\boldsymbol{a} \in\{\boldsymbol{A x}, \boldsymbol{B x}, \boldsymbol{C} \boldsymbol{x}\}^{\perp} \backslash\{\boldsymbol{0}\}, \\
\boldsymbol{b} \in\{\boldsymbol{A} \boldsymbol{y}, \boldsymbol{B} \boldsymbol{y}, \boldsymbol{C} \boldsymbol{y}\}^{\perp} \backslash\{\boldsymbol{0}\}, \\
\boldsymbol{c} \in\{\boldsymbol{A z}, \boldsymbol{B} z, \boldsymbol{C} z\}^{\perp} \backslash\{\boldsymbol{0}\},
\end{array}\right.
$$

or equivalently,

$$
\boldsymbol{a} \odot \boldsymbol{x}, \boldsymbol{b} \odot \boldsymbol{y}, \boldsymbol{c} \odot \boldsymbol{z} \in\{\boldsymbol{A}, \boldsymbol{B}, \boldsymbol{C}\}^{\perp} \backslash\{\boldsymbol{0}\} .
$$

(ii) Up to small perturbations of $\boldsymbol{A}, \boldsymbol{B}, \boldsymbol{C}$, there exist three independent symmetrized rank 1 matrices in the space $\{\boldsymbol{A}, \boldsymbol{B}, \boldsymbol{C}\}^{\perp}$.

Proof. (i) Let $F$ be the cubic function defined by

$$
F(\boldsymbol{x}):=\operatorname{det}(\boldsymbol{A x}, \boldsymbol{B} \boldsymbol{x}, \boldsymbol{C} \boldsymbol{x}) \quad \text { for } \boldsymbol{x} \in \mathbb{R}^{3} .
$$


If $F \equiv 0$ in $\mathbb{R}^{3}$, then condition (7-12) is immediately satisfied. Otherwise, there exists a basis $\left(\boldsymbol{x}_{0}, \boldsymbol{u}_{0}, \boldsymbol{v}_{0}\right)$ of $\mathbb{R}^{3}$ in the nonempty open set $\{F \neq 0\}$. Since we have

$$
F\left(\boldsymbol{x}_{0}+s \boldsymbol{u}_{0}\right) \underset{|s| \rightarrow \infty}{\sim} s^{3} F\left(\boldsymbol{u}_{0}\right) \underset{|s| \rightarrow \infty}{\longrightarrow} \pm \infty,
$$

there exists $s, t \in \mathbb{R} \backslash\{0\}$ such that $\boldsymbol{x}:=\boldsymbol{x}_{0}+s \boldsymbol{u}_{0}$ and $\boldsymbol{y}:=\boldsymbol{x}_{0}+t \boldsymbol{v}_{0}$ are two independent vectors in the set $\{F=0\}$.

First, assume that the set $\{F=0\}$ is not contained in the plane $\operatorname{Span}\{\boldsymbol{x}, \boldsymbol{y}\}$. Then there exists a basis $(\boldsymbol{x}, \boldsymbol{y}, \boldsymbol{z})$ of $\mathbb{R}^{3}$ in the set $\{F=0\}$. Therefore, there exist three vectors $\boldsymbol{a}, \boldsymbol{b}, \boldsymbol{c}$ of $\mathbb{R}^{3}$ satisfying (7-12), or equivalently (7-13).

Now, assume that $\{F=0\} \subset \operatorname{Span}\{\boldsymbol{x}, \boldsymbol{y}\}$. First of all, up to small perturbations we can assume that the matrices $\boldsymbol{A}, \boldsymbol{B}, \boldsymbol{C}$ are invertible. Since $\boldsymbol{B}^{-1} \boldsymbol{C}$ is a $3 \times 3$ real matrix, it has at least a real eigenvalue $\lambda$. The perturbation procedure is now divided into two cases.

First case: The matrix $\boldsymbol{B}^{-1} \boldsymbol{C}$ has two complex conjugate eigenvalues.

Then the eigenspace $\operatorname{Ker}\left(\boldsymbol{B}^{-1} \boldsymbol{C}-\lambda \boldsymbol{I}_{3}\right)$ is a line of $\mathbb{R}^{3}$ spanned by $\boldsymbol{e} \in \mathbb{R}^{3} \backslash\{\boldsymbol{0}\}$. Consider a basis $\left(\boldsymbol{x}_{0}, \boldsymbol{u}_{0}, \boldsymbol{v}_{0}\right)$ of $\mathbb{R}^{3}$ in the set $\{F \neq 0\}$ such that $\left(\boldsymbol{e}, \boldsymbol{x}_{0}, \boldsymbol{u}_{0}\right)$ and $\left(\boldsymbol{e}, \boldsymbol{x}_{0}, \boldsymbol{v}_{0}\right)$ are also two bases of $\mathbb{R}^{3}$. As previously there exist $s, t \in \mathbb{R} \backslash\{0\}$ such that $\boldsymbol{x}:=\boldsymbol{x}_{0}+s \boldsymbol{u}_{0}$ and $\boldsymbol{y}:=\boldsymbol{x}_{0}+t \boldsymbol{v}_{0}$ are two independent vectors of the set $\{F=0\}$. Moreover, since $(\boldsymbol{e}, \boldsymbol{x})$ and $(\boldsymbol{e}, \boldsymbol{y})$ are two families of independent vectors and $\mathbb{R} \boldsymbol{e}$ is the unique real eigenspace of the matrix $\boldsymbol{B}^{-1} \boldsymbol{C}$, we have

$$
\boldsymbol{B} \boldsymbol{x} \times \boldsymbol{C} \boldsymbol{x} \neq \mathbf{0} \text { and } \boldsymbol{B} \boldsymbol{y} \times \boldsymbol{C} \boldsymbol{y} \neq \mathbf{0} .
$$

Now, consider a vector $\boldsymbol{u} \in\{\boldsymbol{x}, \boldsymbol{y}\}^{\perp} \backslash\{\boldsymbol{0}\}$ and the matrix $\boldsymbol{M} \in \mathbb{R}^{3 \times 3}$ defined by

$$
M x=\xi, \quad M y=\eta, \quad M u=0,
$$

where the vectors $\xi, \eta$ will be chosen later. Define for $\tau>0$ the perturbed function

$$
F_{\tau}(\boldsymbol{z}):=\operatorname{det}(\boldsymbol{A} \boldsymbol{z}+\tau \boldsymbol{M} \boldsymbol{z}, \boldsymbol{B} \boldsymbol{z}, \boldsymbol{C} \boldsymbol{z}) \text { for } \boldsymbol{z} \in \mathbb{R}^{3} .
$$

We have

$$
\left\{\begin{array}{l}
F_{\tau}(\boldsymbol{x}+\tau \boldsymbol{u})=\tau \boldsymbol{\xi} \cdot(\boldsymbol{B} \boldsymbol{x} \times \boldsymbol{C} \boldsymbol{x}+\boldsymbol{O}(\tau))+O(\tau), \\
F_{\tau}(\boldsymbol{y}+\tau \boldsymbol{u})=\tau \boldsymbol{\eta} \cdot(\boldsymbol{B} \boldsymbol{y} \times \boldsymbol{C} \boldsymbol{y}+\boldsymbol{O}(\tau))+O(\tau),
\end{array}\right.
$$

where the $\boldsymbol{O}(\tau)$ denote some first-order vectors in $\tau$ and $O(\tau)$ some first-order real numbers in $\tau$ which are independent of $\boldsymbol{\xi}, \boldsymbol{\eta}$. Condition (7-16) then allows us to choose $\boldsymbol{\xi}=\boldsymbol{\xi}_{\tau}$ and $\boldsymbol{\eta}=\boldsymbol{\eta}_{\tau}$ such that $F_{\tau}(\boldsymbol{x}+\tau \boldsymbol{u})=F_{\tau}(\boldsymbol{y}+\tau \boldsymbol{u})=0$. Therefore, since $(\boldsymbol{x}, \boldsymbol{y}, \boldsymbol{u})$ is a basis of $\mathbb{R}^{3},(\boldsymbol{x}, \boldsymbol{x}+\tau \boldsymbol{u}, \boldsymbol{y}+\tau \boldsymbol{u})$ is also a basis of $\mathbb{R}^{3}$, which in addition lies in the set $\left\{F_{\tau}=0\right\}$. This leads us to condition (7-12) with the matrices $\boldsymbol{A}+\tau \boldsymbol{M}, \boldsymbol{B}, \boldsymbol{C}$. 
Second case: The matrix $\boldsymbol{B}^{-1} \boldsymbol{C}$ has only real eigenvalues.

Then there exists a small perturbation $\boldsymbol{C}_{\tau}$ of $\boldsymbol{C}$ such that the perturbed matrix $\boldsymbol{B}^{-1} \boldsymbol{C}_{\tau}$ has three distinct real eigenvalues. Hence, the matrix $\boldsymbol{B}^{-1} \boldsymbol{C}_{\tau}$ admits a basis $(\boldsymbol{x}, \boldsymbol{y}, \boldsymbol{z})$ of eigenvectors, which implies that

$$
\boldsymbol{C}_{\tau} \boldsymbol{x}-\lambda \boldsymbol{B} \boldsymbol{x}=\boldsymbol{C}_{\tau} \boldsymbol{y}-\lambda \boldsymbol{B} \boldsymbol{y}=\boldsymbol{C}_{\tau} z-\lambda \boldsymbol{B} z=\mathbf{0} .
$$

Therefore, the perturbed function

$$
F_{\tau}(\boldsymbol{u}):=\operatorname{det}\left(\boldsymbol{A} \boldsymbol{u}, \boldsymbol{B} \boldsymbol{u}, \boldsymbol{C}_{\tau} \boldsymbol{u}\right) \quad \text { for } \boldsymbol{u} \in \mathbb{R}^{3}
$$

satisfies $F_{\tau}(\boldsymbol{x})=F_{\tau}(\boldsymbol{y})=F_{\tau}(\boldsymbol{z})=0$, which again leads us to condition (7-12) with the matrices $\boldsymbol{A}, \boldsymbol{B}, \boldsymbol{C}_{\tau}$.

(ii) We will distinguish four cases according to whether the following conditions are satisfied by the basis $(\boldsymbol{x}, \boldsymbol{y}, \boldsymbol{z})$ of $\mathbb{R}^{3}$ and the vectors $\boldsymbol{a}, \boldsymbol{b}, \boldsymbol{c} \in \mathbb{R}^{3} \backslash\{0\}$ obtained in step (i):

$$
\left\{\begin{array}{l}
\boldsymbol{a} \in \operatorname{Span}\{\boldsymbol{x}, \boldsymbol{y}\} \cap \operatorname{Span}\{\boldsymbol{x}, \boldsymbol{z}\}, \\
\boldsymbol{b} \in \operatorname{Span}\{\boldsymbol{y}, \boldsymbol{x}\} \cap \operatorname{Span}\{\boldsymbol{y}, \boldsymbol{z}\}, \\
\boldsymbol{c} \in \operatorname{Span}\{\boldsymbol{z}, \boldsymbol{x}\} \cap \operatorname{Span}\{\boldsymbol{z}, \boldsymbol{y}\} .
\end{array}\right.
$$

First case: $\boldsymbol{a}, \boldsymbol{b}$ and $\boldsymbol{c}$ satisfy conditions (7-22).

Then, since $(\boldsymbol{x}, \boldsymbol{y}, \boldsymbol{z})$ is a basis of $\mathbb{R}^{3}$, we have necessarily $\boldsymbol{a} \in \mathbb{R} \boldsymbol{x}, \boldsymbol{b} \in \mathbb{R} \boldsymbol{y}, \boldsymbol{c} \in \mathbb{R} \boldsymbol{z}$. Therefore, $\boldsymbol{x} \odot \boldsymbol{x}, \boldsymbol{y} \odot \boldsymbol{y}, \boldsymbol{z} \odot \boldsymbol{z}$ are clearly three independent matrices of $\{\boldsymbol{A}, \boldsymbol{B}, \boldsymbol{C}\}^{\perp}$. Second case: $\boldsymbol{b}$ and $\boldsymbol{c}$ satisfy conditions (7-22) but $\boldsymbol{a}$ does not.

Then, for example, $(\boldsymbol{a}, \boldsymbol{x}, \boldsymbol{y})$ is a basis of $\mathbb{R}^{3}$, and $\boldsymbol{b} \in \mathbb{R} \boldsymbol{y}, \boldsymbol{c} \in \mathbb{R} \boldsymbol{z}$. Let $\boldsymbol{u} \in\{\boldsymbol{y}, \boldsymbol{z}\}^{\perp} \backslash\{\boldsymbol{0}\}$, and let $\alpha, \beta, \gamma \in \mathbb{R}$ be such that $\alpha \boldsymbol{a} \odot \boldsymbol{x}+\beta \boldsymbol{y} \odot \boldsymbol{y}+\gamma \boldsymbol{z} \odot \boldsymbol{z}=\mathbf{0}$. Multiplying by $\boldsymbol{u}$ we get that $\alpha(\boldsymbol{x} \cdot \boldsymbol{u}) \boldsymbol{a}+\alpha(\boldsymbol{a} \cdot \boldsymbol{u}) \boldsymbol{x}=\mathbf{0}$; hence $\alpha=0$ since $\boldsymbol{x} \cdot \boldsymbol{u} \neq 0$. We deduce immediately that $\beta=\gamma=0$. Therefore, $\boldsymbol{a} \odot \boldsymbol{x}, \boldsymbol{y} \odot \boldsymbol{y}, \boldsymbol{z} \odot \boldsymbol{z}$ are three independent matrices of $\{\boldsymbol{A}, \boldsymbol{B}, \boldsymbol{C}\}^{\perp}$.

Third case: $\boldsymbol{a}$ and $\boldsymbol{b}$ do not satisfy conditions (7-22), with $\boldsymbol{a} \notin \operatorname{Span}\{\boldsymbol{x}, \boldsymbol{y}\}$ and $\boldsymbol{b} \notin \mathbb{R} \boldsymbol{a} \cup \mathbb{R} \boldsymbol{x}$ (respectively $\boldsymbol{a} \notin \operatorname{Span}\{\boldsymbol{x}, \boldsymbol{z}\}$ and $\boldsymbol{c} \notin \mathbb{R} \boldsymbol{a} \cup \mathbb{R} \boldsymbol{x}$ ).

Then $(\boldsymbol{a}, \boldsymbol{x}, \boldsymbol{y})$ is a basis of $\mathbb{R}^{3}$. Let $\boldsymbol{u} \in\{\boldsymbol{x}, \boldsymbol{y}\}^{\perp} \backslash\{\boldsymbol{0}\}$, and let $\alpha, \beta \in \mathbb{R}$ be such that $\alpha \boldsymbol{a} \odot \boldsymbol{x}+\beta \boldsymbol{b} \odot \boldsymbol{y}=\mathbf{0}$. Multiplying by $\boldsymbol{u}$ we get that $\alpha(\boldsymbol{a} \cdot \boldsymbol{u}) \boldsymbol{x}+\beta(\boldsymbol{b} \cdot \boldsymbol{u}) \boldsymbol{y}=\mathbf{0}$; hence $\alpha=0$ since $\boldsymbol{a} \cdot \boldsymbol{u} \neq 0$, and thus $\beta=0$. Therefore, $\boldsymbol{a} \odot \boldsymbol{x}, \boldsymbol{b} \odot \boldsymbol{y}$ are two independent matrices of $\{\boldsymbol{A}, \boldsymbol{B}, \boldsymbol{C}\}^{\perp}$, which have two eigenvalues of opposite sign and one 0 eigenvalue.

Let us prove by contradiction that

$$
\exists t \in \mathbb{R} \backslash\{0\}, \quad \operatorname{det}(\boldsymbol{a} \odot \boldsymbol{x}+t \boldsymbol{b} \odot \boldsymbol{y}) \neq 0 .
$$


Otherwise, for any $t \neq 0$, there exists $\boldsymbol{z}_{t} \in \operatorname{Ker}(\boldsymbol{a} \odot \boldsymbol{x}+t \boldsymbol{b} \odot \boldsymbol{y}) \backslash\{\boldsymbol{0}\}$; hence

$$
\left(\boldsymbol{x} \cdot \boldsymbol{z}_{t}\right) \boldsymbol{a}+\left(\boldsymbol{a} \cdot \boldsymbol{z}_{t}\right) \boldsymbol{x}+t\left(\boldsymbol{y} \cdot \boldsymbol{z}_{t}\right) \boldsymbol{b}+t\left(\boldsymbol{b} \cdot \boldsymbol{z}_{t}\right) \boldsymbol{y}=\mathbf{0} .
$$

Since $(\boldsymbol{a}, \boldsymbol{x}, \boldsymbol{y})$ is a basis of $\mathbb{R}^{3}$ and $\boldsymbol{z}_{t} \neq \mathbf{0}$, we have necessarily $\boldsymbol{y} \cdot \boldsymbol{z}_{t} \neq 0$, which implies that

$$
-\boldsymbol{b}=\frac{\boldsymbol{x} \cdot \boldsymbol{z}_{t}}{t\left(\boldsymbol{y} \cdot \boldsymbol{z}_{t}\right)} \boldsymbol{a}+\frac{\boldsymbol{a} \cdot \boldsymbol{z}_{t}}{t\left(\boldsymbol{y} \cdot \boldsymbol{z}_{t}\right)} \boldsymbol{x}+\frac{\boldsymbol{b} \cdot \boldsymbol{z}_{t}}{\boldsymbol{y} \cdot \boldsymbol{z}_{t}} \boldsymbol{y}=\alpha \boldsymbol{a}+\beta \boldsymbol{x}+\gamma \boldsymbol{y},
$$

where $\alpha, \beta, \gamma$ are independent of $t$, and

$$
(\boldsymbol{x}-\alpha t \boldsymbol{y}) \cdot z_{t}=(\boldsymbol{a}-\beta t \boldsymbol{y}) \cdot \boldsymbol{z}_{t}=(\boldsymbol{b}-\gamma \boldsymbol{y}) \cdot \boldsymbol{z}_{t}=0 .
$$

Since $\boldsymbol{z}_{t} \neq \mathbf{0}$ there exists $\left(p_{t}, q_{t}, r_{t}\right) \in \mathbb{R}^{3} \backslash\{0\}$ such that

$$
\begin{aligned}
p_{t}(\boldsymbol{x}-\alpha t \boldsymbol{y})+q_{t}(\boldsymbol{a}-\beta t \boldsymbol{y})+r_{t}(\boldsymbol{b}-\gamma \boldsymbol{y}) & \\
& =\left(q_{t}-\alpha r_{t}\right) \boldsymbol{a}+\left(p_{t}-\beta r_{t}\right) \boldsymbol{x}-\left(\alpha t p_{t}+\beta t q_{t}+2 \gamma r_{t}\right) \boldsymbol{y}=\mathbf{0},
\end{aligned}
$$

which implies that $q_{t}=\alpha r_{t}, p_{t}=\beta r_{t}$ and $r_{t}(\alpha \beta t+\gamma)=0$. Since $\left(p_{t}, q_{t}, r_{t}\right) \neq \mathbf{0}$, we have $r_{t} \neq 0$ and $\alpha \beta t+\gamma=0$ for any $t \neq 0$; hence $\alpha \beta=0$ and $\gamma=0$. This yields a contradiction between (7-25) and $\boldsymbol{b} \notin \mathbb{R} \boldsymbol{a} \cup \mathbb{R} \boldsymbol{x}$.

By virtue of (7-23) there exist two nonzero real numbers $\alpha \neq \beta$ such that the matrices

$$
\boldsymbol{M}:=\boldsymbol{a} \odot \boldsymbol{x}+\alpha \boldsymbol{b} \odot \boldsymbol{y} \quad \text { and } \quad \boldsymbol{N}:=\boldsymbol{a} \odot \boldsymbol{x}+\beta \boldsymbol{b} \odot \boldsymbol{y}
$$

are invertible. The function $p(t):=\operatorname{det}(\beta \boldsymbol{M}-t \boldsymbol{N})$ is a polynomial of degree 3 whose $\alpha, \beta$ are two distinct roots. Then the polynomial $p(t)$ must change sign by crossing $\alpha$, for example (the conclusion is similar for $\beta$ ). Let $\lambda_{1}(t) \leq \lambda_{2}(t) \leq \lambda_{3}(t)$ be the well-ordered eigenvalues of the symmetric matrix $\beta \boldsymbol{M}-t \boldsymbol{N}$. Since the vectors $\boldsymbol{a}, \boldsymbol{x}$ are independent, $\boldsymbol{a} \odot \boldsymbol{x}$ has two eigenvalues of opposite sign and one 0 eigenvalue; hence $\lambda_{1}(\alpha)<\lambda_{2}(\alpha)=0<\lambda_{3}(\alpha)$.

Now, let $\boldsymbol{P}_{\tau}$ for a small $\tau>0$ be a symmetric matrix in the space $\{\boldsymbol{A}, \boldsymbol{B}, \boldsymbol{C}\}^{\perp}$, such that $\left|\boldsymbol{P}_{\tau}-\boldsymbol{a} \odot \boldsymbol{x}\right|=O(\tau)$, and such that the three matrices $\boldsymbol{a} \odot \boldsymbol{x}, \boldsymbol{b} \odot \boldsymbol{y}, \boldsymbol{P}_{\tau}$ are independent (note that the dimension of $\{\boldsymbol{A}, \boldsymbol{B}, \boldsymbol{C}\}^{\perp}$ is $\geq 3$ ). Define the two perturbed matrices

$$
\boldsymbol{M}_{\tau}:=\boldsymbol{P}_{\tau}+\alpha \boldsymbol{b} \odot \boldsymbol{y} \quad \text { and } \quad \boldsymbol{N}_{\tau}:=\boldsymbol{P}_{\tau}+\beta \boldsymbol{b} \odot \boldsymbol{y} .
$$

Since the well-ordered eigenvalues of a real symmetric matrix $S$ are Lipschitzcontinuous with respect to $S$ (see, e.g., [Ciarlet 1989], Theorem 2.3-2), the eigenvalues $\lambda_{1}^{\tau}(t) \leq \lambda_{2}^{\tau}(t) \leq \lambda_{3}^{\tau}(t)$ of $\beta \boldsymbol{M}_{\tau}-t \boldsymbol{N}_{\tau}$ converge uniformly as $\tau \rightarrow 0$ to the eigenvalues $\lambda_{1}(t) \leq \lambda_{2}(t) \leq \lambda_{3}(t)$ of $\beta \boldsymbol{M}-t \boldsymbol{N}$, with respect to $t$ in a neighborhood of $\alpha$. Hence, for $\tau>0$ small enough, there exist $\alpha_{\tau}$ close to $\alpha$ such that $\alpha_{\tau} \neq \beta$ and 
$\lambda_{1}^{\tau}\left(\alpha_{\tau}\right)<\lambda_{2}^{\tau}\left(\alpha_{\tau}\right)=0<\lambda_{3}^{\tau}\left(\alpha_{\tau}\right)$. Then by (7-29) there exist $\boldsymbol{c}_{\tau}, \boldsymbol{z}_{\tau} \in \mathbb{R}^{3}$ such that

$$
\beta \boldsymbol{M}_{\tau}-\alpha_{\tau} \boldsymbol{N}_{\tau}=\boldsymbol{c}_{\tau} \odot \boldsymbol{z}_{\tau}=\left(\beta-\alpha_{\tau}\right) \boldsymbol{P}_{\tau}+\beta\left(\alpha-\alpha_{\tau}\right) \boldsymbol{b} \odot \boldsymbol{y} \text {, with } \beta-\alpha_{\tau} \neq 0 .
$$

Therefore, $\boldsymbol{a} \odot \boldsymbol{x}, \boldsymbol{b} \odot \boldsymbol{y}, \boldsymbol{c}_{\tau} \odot \boldsymbol{z}_{\tau}$ are three independent symmetrized rank 1 matrices in the space $\{\boldsymbol{A}, \boldsymbol{B}, \boldsymbol{C}\}^{\perp}$.

Fourth case: $\boldsymbol{a}$ and $\boldsymbol{b}$ do not satisfy conditions (7-22), with $\boldsymbol{a} \notin \operatorname{Span}\{\boldsymbol{x}, \boldsymbol{y}\}$ and $\boldsymbol{b} \in \mathbb{R} \boldsymbol{a} \cup \mathbb{R} \boldsymbol{x}$ (respectively $\boldsymbol{a} \notin \operatorname{Span}\{\boldsymbol{x}, \boldsymbol{z}\}$ and $\boldsymbol{c} \in \mathbb{R} \boldsymbol{a} \cup \mathbb{R} \boldsymbol{x}$ ).

For example, we have $\boldsymbol{b} \in \mathbb{R} \boldsymbol{a}$. We thus start from the matrices $\boldsymbol{a} \odot \boldsymbol{x}$ and $\boldsymbol{a} \odot \boldsymbol{y}$ in the space $\{\boldsymbol{A}, \boldsymbol{B}, \boldsymbol{C}\}^{\perp}$, where $(\boldsymbol{a}, \boldsymbol{x}, \boldsymbol{y})$ is a basis of $\mathbb{R}^{3}$. We will consider a perturbation of $\boldsymbol{A}, \boldsymbol{B}, \boldsymbol{C}$ for leading us to the third case.

Let $\boldsymbol{t} \in\{\boldsymbol{a}, \boldsymbol{x}\}^{\perp} \backslash\{\boldsymbol{0}\}$, let $\boldsymbol{d} \in \mathbb{R}^{3} \backslash(\mathbb{R} \boldsymbol{a}+\mathbb{R} \boldsymbol{x})$, and consider, for a small $\tau>0$, the perturbed vector $\boldsymbol{b}_{\tau}:=\boldsymbol{a}+\tau \boldsymbol{d} \notin \mathbb{R} \boldsymbol{a} \cup \mathbb{R} \boldsymbol{x}$ and the perturbed matrices

$$
\boldsymbol{A}_{\tau}:=\boldsymbol{A}+\tau \boldsymbol{t} \odot \boldsymbol{u}_{\tau}, \quad \boldsymbol{B}_{\tau}:=\boldsymbol{B}+\tau \boldsymbol{t} \odot \boldsymbol{v}_{\tau}, \quad \boldsymbol{C}_{\tau}:=\boldsymbol{C}+\tau \boldsymbol{t} \odot \boldsymbol{w}_{\tau},
$$

where the vectors $\boldsymbol{u}_{\tau}, \boldsymbol{v}_{\tau}, \boldsymbol{w}_{\tau}$ will be chosen later. Clearly, $\boldsymbol{a} \odot \boldsymbol{x} \in\left\{\boldsymbol{A}_{\tau}, \boldsymbol{B}_{\tau}, \boldsymbol{C}_{\tau}\right\}^{\perp}$. On the other hand, we have

$$
\boldsymbol{A}_{\tau}: \boldsymbol{b}_{\tau} \odot \boldsymbol{y}=\tau\left(\boldsymbol{A}: \boldsymbol{d} \odot \boldsymbol{y}+\boldsymbol{t} \odot \boldsymbol{u}_{\tau}: \boldsymbol{a} \odot \boldsymbol{y}+\tau \boldsymbol{t} \odot \boldsymbol{u}_{\tau}: \boldsymbol{d} \odot \boldsymbol{y}\right) .
$$

Since $2 \boldsymbol{t} \odot \boldsymbol{u}_{\tau}: \boldsymbol{a} \odot \boldsymbol{y}=(\boldsymbol{t} \cdot \boldsymbol{y}) \boldsymbol{a} \cdot \boldsymbol{u}_{\tau}$ with $\boldsymbol{t} \cdot \boldsymbol{y} \neq 0$, we can choose $\boldsymbol{u}_{\tau}=\boldsymbol{O}(1)$ with respect to $\tau$ such that $\boldsymbol{A}_{\tau}: \boldsymbol{b}_{\tau} \odot \boldsymbol{y}=0$. Hence, choosing $\boldsymbol{v}_{\tau}$ and $\boldsymbol{w}_{\tau}$ similarly, we get that $\boldsymbol{b}_{\tau} \odot \boldsymbol{y} \in\left\{\boldsymbol{A}_{\tau}, \boldsymbol{B}_{\tau}, \boldsymbol{C}_{\tau}\right\}^{\perp}$. Therefore, the vectors $\boldsymbol{a}, \boldsymbol{b}_{\tau}, \boldsymbol{x}, \boldsymbol{y}$ satisfy the conditions of the third case with the perturbed matrices $\boldsymbol{A}_{\tau}, \boldsymbol{B}_{\tau}, \boldsymbol{C}_{\tau}$.

\section{Constructing suitable multimode materials for the wall microstructure}

Let us specify the construction of the desired multimode materials in two dimensions and then move to three dimensions. We begin by constructing bimode materials that can only support one stress. One could use the fourth-rank laminate structure described in detail in Section 30.7 of [Milton 2002]. The analysis would then be essentially a repeat of that analysis, which builds the appropriate trial stress and strain fields at each length scale. The key feature is that these trial fields need to be chosen so the trial stress associated with the average stress $\sigma^{0}$ we want to achieve at the macroscopic scale is concentrated entirely in phase 1 (apart from boundary layers that we ignore, whose contribution to the energy vanishes in the homogenization limit), and so the trial strain associated with an average strain that is orthogonal to $\sigma^{0}$ is concentrated entirely in phase 2 .

Rather than doing this, it is more instructive to build trial stress and strain fields that are concentrated in phase 1 and phase 2, respectively, for the honeycomb and inverted honeycomb bimode structures of Figure 6, as the ideas here carry over to pentamode materials. The trial stress is easy. It is taken to be macroscopically 

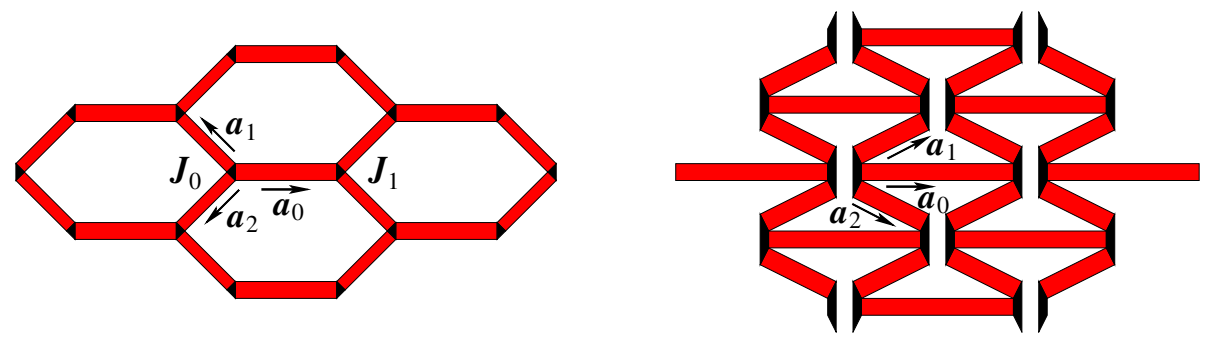

Figure 6. 2-dimensional bimode materials that can only support one average stress field $\sigma^{0}$, and which are easily compliant to any strain orthogonal to $\sigma^{0}$. Here the red struts are laminates of the two phases with the interfaces in the laminate parallel to the direction of the struts. The geometry on the left is appropriate if $\operatorname{det} \sigma^{0}>0$, the geometry on the right is appropriate if $\operatorname{det} \sigma^{0}<0$, and if $\operatorname{det} \sigma^{0}=0$ it suffices to use a simple laminate with the layer surfaces perpendicular to the null vector of $\sigma^{0}$.

constant with a value $\alpha_{i} \boldsymbol{a}_{i} \boldsymbol{a}_{i}^{T}$ in each strut which is parallel to the unit vector $\boldsymbol{a}_{i}$ in Figure 7. Let $w_{i}$ denote the width of the strut parallel to $\boldsymbol{a}_{i}$, for $i=0,1,2$. Since the net "force" on the black junction regions in the top left and top right of Figure 7 must be zero, we obtain

$$
0=-\sum_{k=0}^{2} w_{i}\left(\alpha_{i} \boldsymbol{a}_{i} \boldsymbol{a}_{i}^{T}\right) \boldsymbol{a}_{i}=-\sum_{k=0}^{2} w_{i} \alpha_{i} \boldsymbol{a}_{i} .
$$

Since $w_{1}=w_{2}$ and $\boldsymbol{a}_{0}$ points in the horizontal direction, while $\boldsymbol{a}_{1}$ and $\boldsymbol{a}_{2}$ have the same horizontal component and equal but opposite vertical components, we get

$$
\alpha_{1}=\alpha_{2}=-w_{0} \alpha_{0} /\left[2 w_{1}\left(\boldsymbol{a}_{1} \cdot \boldsymbol{a}_{2}\right)\right] .
$$

The symmetry of the trial stress field implies there is no associated torque acting on the junction regions. The trial stress in the junction regions is really not that important. One choice is the stress field that satisfies the elasticity equations appropriate to phase 1 filling the junction region when constant tractions act on the three sides. The average value of the trial stress does not depend on the choice of trial stress in the junctions. Indeed, since $\nabla \cdot(\sigma)=0$ it follows from integration by parts of $\nabla \cdot(\boldsymbol{\sigma} \boldsymbol{x})$ (where $\boldsymbol{\sigma} \boldsymbol{x}$ is a third-order tensor) that

$$
\int_{\Omega} \boldsymbol{\sigma} d \boldsymbol{x}=\int_{\partial \Omega} \boldsymbol{t} \boldsymbol{x}^{T} d S, \quad \text { where } \boldsymbol{t}=\boldsymbol{\sigma} \boldsymbol{n} \text { is the surface traction, }
$$

in which $\Omega$ is any region with boundary $\partial \Omega$. For example, the boundary of $\Omega$ could be the outermost boundary of the shape in the top left or top right of Figure 7, where we include the dashed lines as part of the boundary. 

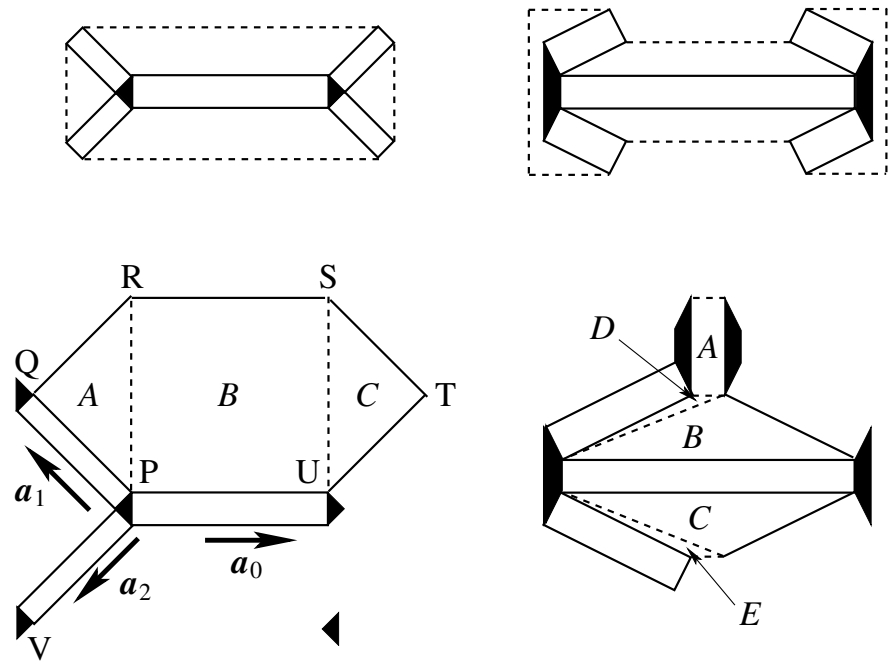

Figure 7. The honeycomb structure of Figure 6 (left) can be taken to have the unit cell shown at top left. Similarly the inverted honeycomb structure of Figure 6 (right) can be taken to have the unit cell shown at top right. The space outside the struts and junction regions (which is occupied by phase 2) has been triangulated with boundaries marked by the dashed lines to make the construction of the trial stress fields easy.

In passing, we remark that if $\sigma^{0}$ is proportional to the identity matrix, then the microstructure of Figure 7 (top left) resembles a Sigmund microstructure (see the last subfigure in Figure 2 in [Sigmund 2000]). However, we do not require the tuning of layer widths in the struts that makes his structure optimal. Suboptimal structures are perfectly fine in the walls, since the walls ultimately occupy a vanishingly small volume fraction in the final material.

To obtain a trial easy strain it suffices to specify the trial displacement in the unit cell. We only choose motions so the junction regions (triangular in Figure 7 (bottom left) and quadrilateral in Figure 7 (bottom right)) undergo rigid body translations, so there is no strain inside them. Thus associated with Figure 7 (bottom left) one can clearly identify two independent macroscopic modes of motion. The first is where the line RS moves vertically upwards while the line PU remains fixed, and $\mathrm{Q}$ and T move in such a way that the lengths QR, QP, TS, and TU remain equal and preserved in length. One can choose the displacement to be linear in each of the three regions $A, B$, and $C$ so that it matches the displacement on the boundary. The second is where the line RS moves horizontally while the line PU remains fixed, and $\mathrm{Q}$ and $\mathrm{T}$ move in such a way that the lengths $\mathrm{QR}, \mathrm{QP}, \mathrm{TS}$, and TU remain equal and preserved in length. In either case inside the horizontal laminate arm there is no 

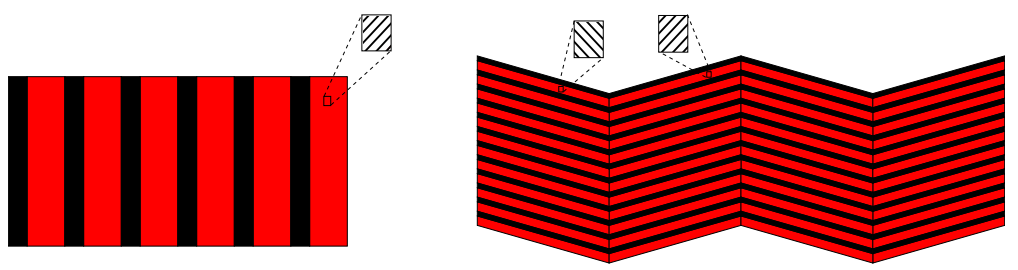

Figure 8. 2-dimensional unimode materials that are easily compliant to one average strain field $\epsilon^{0}$, and which can support any stress orthogonal to $\epsilon^{0}$. In both, the red region represents a laminate as indicated by the inserts. The second-rank laminate geometry on the left is appropriate if $\operatorname{det} \epsilon^{0}<0$ and the third-rank geometry on the right is appropriate for any $\epsilon^{0}$.

strain, while inside the inclined laminate arms there is an infinitesimal shear so the junction at $\mathrm{P}$ remains fixed, while the junction at $\mathrm{Q}$ moves perpendicular to $\boldsymbol{a}_{1}$ and the junction at $\mathrm{V}$ moves perpendicular to $\boldsymbol{a}_{2}$. We also note that there is also an easy microscopic motion which results in no macroscopic motion. Define the center of each triangular junction to be the point which is at the junction of the perpendicular bisector of the three faces. Then if all the triangular junctions undergo the same infinitesimal rotation about these centers while the laminate material in the struts shears at the same time, it will cost very little energy. The trial strain field is bounded and nonzero only in phase 2 , and therefore the associated upper bound on the elastic energy scales in proportion to $\delta$.

The situation in Figure 7 (bottom right) is basically similar. The two black quadrilateral junction regions at the bottom of the figure can remain fixed. Then one mode is the symmetric one, where the region $A$ undergoes uniaxial compression in the horizontal direction and at the same time moves downwards. The second is where the region $A$ undergoes pure shear, so the junction on the left side of it moves up, while the right side moves down. The strain field can be taken constant in the regions $A, B, C, D$, and $E$, and in the inclined laminate strut arms is also constant and corresponds to pure shear. These strains are easily determined from the value of the trial displacement field at the boundaries of each region. Again, the trial strain field is bounded and nonzero only in phase 2 , and therefore the associated upper bound on the elastic energy scales in proportion to $\delta$.

The structures of Figure 8 give suitable 2-dimensional unimode materials. We will not specify the appropriate trial stress and strain fields which prove that these structures have the desired elastic behavior, as they are exactly the same as those given in Section 30.6 of [Milton 2002].

We now describe the pentamodes and the trial fields in them. Given four vectors $\boldsymbol{a}_{0}, \boldsymbol{a}_{1}, \boldsymbol{a}_{2}$, and $\boldsymbol{a}_{4}$ (no longer required to be unit vectors) we position a point $\mathrm{P}$ at 


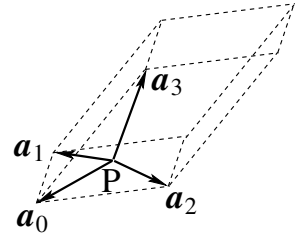

(a)

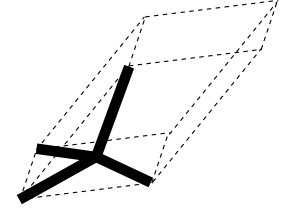

(b)

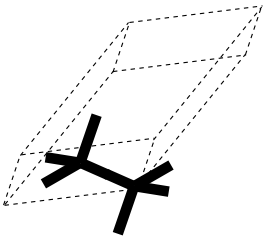

(c)

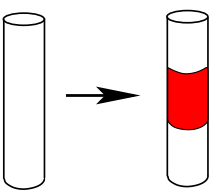

(d)

Figure 9. The procedure for constructing the desired pentamodes. In (d) a shearable section is inserted into each strut. This section, shown in red, has the structure of parallel square fibers, as illustrated in Figure 10, with the fibers aligned parallel to the strut.

the origin, and join $\mathrm{P}$ to the four points $\boldsymbol{x}=\boldsymbol{a}_{i}$ for $i=0,1,2,3$, with four infinitesimally thin rods, as in Figure 9(a). We then take as our unit cell of periodicity the parallelepiped with the eight points $x=a_{i}, x=a_{1}+a_{2}+a_{3}-a_{i}-a_{0}$ for $i=0,1,2,3$ (the three vectors $\boldsymbol{v}_{i}=\boldsymbol{a}_{i}-\boldsymbol{a}_{0}$ for $i=1,2,3$ are the primitive lattice vectors). We require that $\boldsymbol{a}_{0}, \boldsymbol{a}_{1}, \boldsymbol{a}_{2}, \boldsymbol{a}_{4}$ be chosen so $\mathrm{P}$ lies within this parallelepiped. After periodically extending the rod structure (with rods joining $k_{1} \boldsymbol{v}_{1}+k_{2} \boldsymbol{v}_{1}+k_{3} \boldsymbol{v}_{1}$ with the four points $k_{1} \boldsymbol{v}_{1}+k_{2} \boldsymbol{v}_{1}+k_{3} \boldsymbol{v}_{1}+a_{i}$ for $i=0,1,2,3$, for any integers $k_{1}, k_{2}$, and $k_{3}$ ), we then coat this periodic rod structure with phase 1 , as illustrated in Figure 9(b), so that any point $\boldsymbol{x}$ is in phase 1 if and only if it is within a distance $r$ of the rod structure. Here $r$ should be chosen appropriately small so that the coatings of each rod contain a cylindrical section that we refer to as a strut. Figure 9(b) is misleading as it suggests that the unit cell only contains one junction region. The true structure which should be periodically repeated (by making copies shifted by vectors $k_{1} \boldsymbol{v}_{1}+k_{2} \boldsymbol{v}_{1}+k_{3} \boldsymbol{v}_{1}$ for all combinations of integers $k_{1}, k_{2}$, and $k_{3}$ ) is shown in Figure 9(c) and contains the junction of Figure 9(b) plus the one obtained by inverting it under the transformation $\boldsymbol{x} \rightarrow-\boldsymbol{x}$. The final step, illustrated in Figure 9(d), is to take a cylindrical subsection of each cylindrical section between junctions and replace it with a pentamode material that supports any stress proportional to $\boldsymbol{a}_{i} \boldsymbol{a}_{i}^{T}$. It is convenient to take end faces of the cylindrical subsection to be perpendicular to the cylinder axis, i.e., perpendicular to the vector $\boldsymbol{a}_{i}$ that is parallel to the cylinder axis. Now we define the junction regions to be those connected regions of phase 1 that are bounded by the cylindrical subsections.

To obtain the trial stress field, we first solve for the tensions in the rods of Figure 9(a) when the rods are completely rigid and supporting a stress. These are found just by balance of forces at the junctions. If the rods parallel to $\boldsymbol{a}_{i}$ have a tension $T_{i}$ (which could be negative) then we take in the cylindrical subsection of the corresponding strut of the final pentamode a trial stress field $T_{i} \boldsymbol{a}_{i} \boldsymbol{a}_{i}^{T} /\left(\left|\boldsymbol{a}_{i}\right|^{2} \pi r^{2}\right)$ giving rise to a net force $T_{i}$ pulling (pushing if $T_{i}$ is negative) on the adjacent junction regions. Inside the junction region we take a stress field that satisfies the 

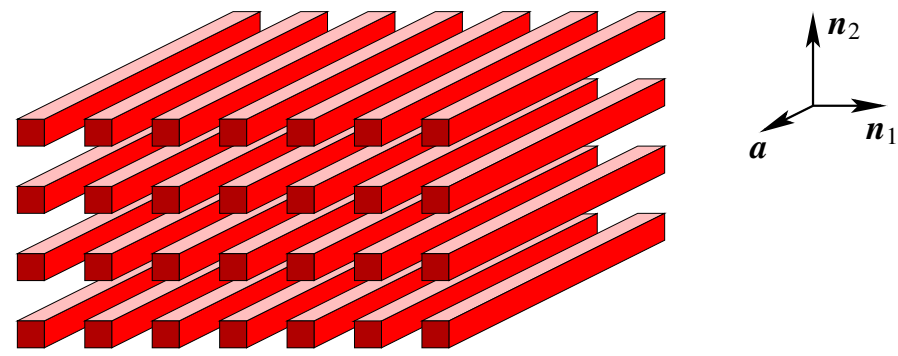

Figure 10. A detailed view of the square beam array microstructure which is used as the easily shearable section in the pentamode cylindrical struts. The vector $\boldsymbol{a}$ is chosen to be one of the four vectors $\boldsymbol{a}_{k}$ for $k=0,1,2,3$, as appropriate to each pentamode strut orientated parallel to $\boldsymbol{a}_{k}$. The square beams can support tension (or compression) in the direction of the beam, and in particular can support a constant macroscopic stress $\boldsymbol{\sigma}_{k}^{B}=\alpha_{k} \boldsymbol{a}_{k} \boldsymbol{a}_{k}^{T}$. As we are working in the framework of linear elasticity, we ignore the very real possibility that the beams will buckle.

elasticity equations appropriate to phase 1 filling the junction region when constant tractions $T_{i} /\left(\pi r^{2}\right)$ act on the four disks that border the cylindrical subsections, and there are no forces on the remaining surface of the junction regions.

Obtaining appropriate trial strain fields is also not too difficult. We first consider an infinitesimal motion that the rod model with Figure 9(a) as the unit cell can undergo when the rods are rigid but the pin junctions are flexible. Then in the final pentamode the junction regions are taken to undergo a rigid body translation which is the same as that of the corresponding pin junction in the rod model. The cylindrical subsections undergo appropriate shears to ensure continuity of the displacement. The trial displacement in the remaining multiconnected region of phase 2 bordered by the junction regions and the cylindrical subsection can be somewhat arbitrary, and is not really important. One could take it as the solution for the displacement field when phase 2 has some nonzero elastic moduli, and the displacement at the boundary of the junctions and cylindrical subsections matches that of the trial field just specified. The trial strain field is bounded and nonzero only in phase 2 , and therefore the associated upper bound on the elastic energy scales in proportion to $\delta$.

It is clear from the choice of these trial stress and strain fields that the macroscopic stress the material supports and the easy motions it permits are exactly the same as those for the ideal model with rods and pin junctions that has the unit cell pictured in Figure 9(a), and which provided the basis for our construction. That this structure can support any desired average stress, and only that average stress, is then a direct consequence of the analysis in Section 5.2 of [Milton and Cherkaev 1995]. 

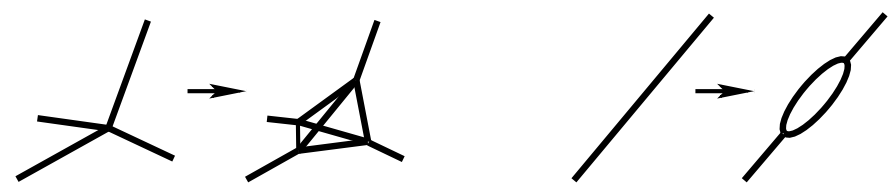

Figure 11. Some of the replacements that are needed to obtain desired unimode, bimode, trimode, or quadramode materials.

To obtain any desired unimode, bimode, trimode, or quadramode material, having respectively $p=1,2,3,4$ independent easy modes of deformation, and supporting respectively $6-p$ applied stresses $\sigma_{j}^{0}$ for $j=1, \ldots, 6-p$, we follow the prescription given by Milton and Cherkaev [1995]. That is, we superimpose, one at a time, $6-p$ pentamode structures, each supporting one of the stresses $\sigma_{j}^{0}$, with struts which are sufficiently thin to ensure that one can (with appropriate modification specified below) superimpose the structures without collision. When doing this superimposition we first remove phase 2 and shift the lattice structures to try to avoid unwanted intersections of phase 1. This may not always be possible, so in the event two vertices clash we make the replacement in Figure 11 (left) in one of the structures (which may of course then cause additional unwanted intersections of the struts). Then if two (or more) struts intersect we make the replacement in Figure 11 (right) in all but one of the struts (which then passes through each hole). The remaining possibility we want to avoid is that two pentamode struts are parallel and intersect when we superimpose the structures. Due to the freedom in the choice of the $\boldsymbol{a}_{k}$ that give a desired $\boldsymbol{\sigma}_{j}^{0}$, we can always choose our $6-p$ pentamode structures to avoid such clashes. Finally, the shearable section in each pentamode strut should be placed in a section that has not been modified, so it still is parallel to one of the $\boldsymbol{a}_{k}$. At the very end any remaining space that is not filled by phase 1 should be filled by the extremely compliant phase 2 .

\section{Continuity of the energy functions}

It follows from the preceding analysis that we can determine the three energy functions

$$
\begin{aligned}
& W_{f}^{3}\left(\boldsymbol{\sigma}_{1}^{0}, \boldsymbol{\sigma}_{2}^{0}, \boldsymbol{\sigma}_{3}^{0}, \boldsymbol{\epsilon}_{1}^{0}, \boldsymbol{\epsilon}_{2}^{0}, \boldsymbol{\epsilon}_{3}^{0}\right), \\
& W_{f}^{4}\left(\boldsymbol{\sigma}_{1}^{0}, \boldsymbol{\sigma}_{2}^{0}, \boldsymbol{\epsilon}_{1}^{0}, \boldsymbol{\epsilon}_{2}^{0}, \boldsymbol{\epsilon}_{3}^{0}, \boldsymbol{\epsilon}_{4}^{0}\right), \\
& W_{f}^{5}\left(\boldsymbol{\sigma}_{1}^{0}, \boldsymbol{\epsilon}_{1}^{0}, \boldsymbol{\epsilon}_{2}^{0}, \boldsymbol{\epsilon}_{3}^{0}, \boldsymbol{\epsilon}_{4}^{0}, \boldsymbol{\epsilon}_{5}^{0}\right)
\end{aligned}
$$

in the limit $\delta \rightarrow 0$ for almost all combinations of applied fields. Here we establish that these energy functions are continuous functions of the applied fields in the limit $\delta \rightarrow 0$, and therefore we obtain expressions for the energy functions for all combinations of applied fields in this limit. 
Recall that the set $G U_{f}$ is characterized by its $W$-transform. For example, part of it is described by the function

$$
W_{f}^{4}\left(\boldsymbol{\sigma}_{1}^{0}, \boldsymbol{\sigma}_{2}^{0}, \boldsymbol{\epsilon}_{1}^{0}, \boldsymbol{\epsilon}_{2}^{0}, \boldsymbol{\epsilon}_{3}^{0}, \boldsymbol{\epsilon}_{4}^{0}\right)=\min _{\boldsymbol{C}_{*} \in G U_{f}}\left[\sum_{i=1}^{4} \boldsymbol{\epsilon}_{i}^{0}: \boldsymbol{C}_{*} \boldsymbol{\epsilon}_{i}^{0}+\sum_{j=1}^{2} \boldsymbol{\sigma}_{j}^{0}: \boldsymbol{C}_{*}^{-1} \boldsymbol{\sigma}_{j}^{0}\right] .
$$

Here we want to show that such energy functions are continuous in their arguments. Let the tensor $\boldsymbol{C}_{*}\left(\boldsymbol{\sigma}_{1}^{0}, \boldsymbol{\sigma}_{2}^{0}, \boldsymbol{\epsilon}_{1}^{0}, \boldsymbol{\epsilon}_{2}^{0}, \boldsymbol{\epsilon}_{3}^{0}, \boldsymbol{\epsilon}_{4}^{0}\right)$ be a minimizer of (9-1), and suppose we perturb the applied stress fields $\sigma_{j}^{0}$ by $\delta \sigma_{j}^{0}$ and the applied strain fields $\epsilon_{i}^{0}$ by $\delta \epsilon_{i}^{0}$. Now consider the walled material with a geometry described by the characteristic function

$$
\chi_{w}(\boldsymbol{x})=\prod_{k=1}^{3}\left(1-H_{\epsilon^{\prime}}\left(\boldsymbol{x} \cdot \boldsymbol{n}_{k}\right)\right)
$$

where $\boldsymbol{n}_{1}, \boldsymbol{n}_{2}$, and $\boldsymbol{n}_{3}$ are the three orthogonal unit vectors

$$
\boldsymbol{n}_{1}=\left(\begin{array}{l}
1 \\
0 \\
0
\end{array}\right), \quad \boldsymbol{n}_{2}=\left(\begin{array}{l}
0 \\
1 \\
0
\end{array}\right), \quad \boldsymbol{n}_{3}=\left(\begin{array}{l}
0 \\
0 \\
1
\end{array}\right)
$$

and $\epsilon^{\prime}$ is a small parameter that gives the thickness of the walls. Inside the walls, where $\chi_{w}(\boldsymbol{x})=0$, we put an isotropic composite of phase 1 and phase 2 , mixed in the proportions $f$ and $1-f$ with isotropic effective elasticity tensor $\boldsymbol{C}\left(\kappa_{0}, \mu_{0}\right)$, where $\kappa_{0}$ is the effective bulk modulus and $\mu_{0}$ is the effective shear modulus, which are assumed to have nonzero limits as $\delta \rightarrow 0$. (The isotropic composite could consist of islands of void surrounded by phase 1.) Outside the walls, where $\chi_{w}(\boldsymbol{x})=1$, we put the material that has an effective tensor

$$
\boldsymbol{C}_{*}^{1}=\boldsymbol{C}_{*}\left(\sigma_{1}^{0}, \sigma_{2}^{0}, \epsilon_{1}^{0}, \epsilon_{2}^{0}, \epsilon_{3}^{0}, \epsilon_{4}^{0}\right) .
$$

Let $\boldsymbol{C}_{*}^{\prime}$ be the effective tensor of the composite. We have the variational principle

$$
\begin{aligned}
\sum_{i=1}^{4}\left(\boldsymbol{\epsilon}_{i}^{0}+\delta \boldsymbol{\epsilon}_{i}^{0}\right): \boldsymbol{C}_{*}^{\prime}\left(\boldsymbol{\epsilon}_{i}^{0}+\delta \boldsymbol{\epsilon}_{i}^{0}\right)+\sum_{j=1}^{2}\left(\boldsymbol{\sigma}_{j}^{0}+\delta \boldsymbol{\sigma}_{j}^{0}\right):\left(\boldsymbol{C}_{*}^{\prime}\right)^{-1}\left(\boldsymbol{\sigma}_{j}^{0}+\delta \boldsymbol{\sigma}_{j}^{0}\right) \\
=\min _{\underline{\boldsymbol{\epsilon}}_{1}, \underline{\epsilon}_{2}, \underline{\epsilon}_{3}, \underline{\epsilon}_{4}, \underline{\boldsymbol{\sigma}}_{1}, \underline{\boldsymbol{\sigma}}_{2}}\left\langle\sum_{i=1}^{4} \underline{\boldsymbol{\epsilon}}_{i}(\boldsymbol{x}):\left[\chi_{w}(\boldsymbol{x}) \boldsymbol{C}_{*}^{1}+\left(1-\chi_{w}(\boldsymbol{x})\right) \boldsymbol{C}\left(\kappa_{0}, \mu_{0}\right)\right] \underline{\boldsymbol{\epsilon}}_{i}(\boldsymbol{x})\right. \\
\left.+\sum_{j=1}^{2} \underline{\boldsymbol{\sigma}}_{j}(\boldsymbol{x}):\left[\chi_{w}(\boldsymbol{x}) \boldsymbol{C}_{*}^{1}+\left(1-\chi_{w}(\boldsymbol{x})\right) \boldsymbol{C}\left(\kappa_{0}, \mu_{0}\right)\right]^{-1} \underline{\boldsymbol{\sigma}}_{j}(\boldsymbol{x})\right),
\end{aligned}
$$

where the minimum is over fields subject to the appropriate average values and differential constraints. We choose constant trial strain fields

$$
\underline{\boldsymbol{\epsilon}}_{i}(\boldsymbol{x})=\boldsymbol{\epsilon}_{i}^{0}+\delta \boldsymbol{\epsilon}_{i}^{0}, \quad i=1,2,3,4,
$$


and trial stress fields

$$
\underline{\boldsymbol{\sigma}}_{j}(\boldsymbol{x})=\boldsymbol{\sigma}_{j}^{0}+\delta \underline{\boldsymbol{\sigma}}_{j}(\boldsymbol{x}), \quad j=1,2,
$$

where $\delta \underline{\boldsymbol{\sigma}}_{j}(\boldsymbol{x})$ has average value $\delta \boldsymbol{\sigma}_{j}^{0}$ and is concentrated in the walls. Specifically, if $\left\{\delta \sigma_{j}^{0}\right\}_{k \ell}$ denote the matrix elements of $\delta \sigma_{j}^{0}$, and letting

$$
\begin{aligned}
& \delta \boldsymbol{\sigma}_{j}^{1}=\left(\begin{array}{ccc}
0 & 0 & 0 \\
0 & 0 & \left\{\delta \boldsymbol{\sigma}_{j}^{0}\right\}_{23} \\
0 & \left\{\delta \boldsymbol{\sigma}_{j}^{0}\right\}_{32} & \left\{\delta \boldsymbol{\sigma}_{j}^{0}\right\}_{33}
\end{array}\right), \\
& \delta \boldsymbol{\sigma}_{j}^{2}=\left(\begin{array}{ccc}
\left\{\delta \boldsymbol{\sigma}_{j}^{0}\right\}_{11} & 0 & \left\{\delta \boldsymbol{\sigma}_{j}^{0}\right\}_{13} \\
0 & 0 & 0 \\
\left\{\delta \boldsymbol{\sigma}_{j}^{0}\right\}_{31} & 0 & 0
\end{array}\right), \\
& \delta \boldsymbol{\sigma}_{j}^{3}=\left(\begin{array}{ccc}
0 & \left\{\delta \boldsymbol{\sigma}_{j}^{0}\right\}_{12} & 0 \\
\left\{\delta \boldsymbol{\sigma}_{j}^{0}\right\}_{21} & \left\{\delta \boldsymbol{\sigma}_{j}^{0}\right\}_{22} & 0 \\
0 & 0 & 0
\end{array}\right),
\end{aligned}
$$

then we choose

$$
\delta \underline{\boldsymbol{\sigma}}_{j}(\boldsymbol{x})=\sum_{k=1}^{3} \delta \boldsymbol{\sigma}_{j}^{k} H_{\epsilon^{\prime}}\left(\boldsymbol{x} \cdot \boldsymbol{n}_{k}\right) / \epsilon^{\prime},
$$

which has the required average value $\delta \sigma_{j}^{0}$ and satisfies the differential constraints appropriate to a stress field because $\delta \boldsymbol{\sigma}_{j}^{k} \boldsymbol{n}_{k}=0$.

Hence, there exist positive constants $\alpha$ and $\beta$ such that for sufficiently small $\epsilon^{\prime}$ and for sufficiently small variations $\delta \sigma_{j}^{0}$ and $\delta \boldsymbol{\epsilon}_{i}^{0}$ in the applied fields, we have

$$
\begin{aligned}
\left\langle\sum_{i=1}^{4} \underline{\boldsymbol{\epsilon}}_{i}(\boldsymbol{x}):\left[\chi_{w}(\boldsymbol{x}) \boldsymbol{C}_{*}^{1}+\left(1-\chi_{w}(\boldsymbol{x})\right) \boldsymbol{C}\left(\kappa_{0}, \mu_{0}\right)\right] \underline{\boldsymbol{\epsilon}}_{i}(\boldsymbol{x})\right. \\
\left.+\sum_{j=1}^{2} \underline{\boldsymbol{\sigma}}_{j}(\boldsymbol{x}):\left[\chi_{w}(\boldsymbol{x}) \boldsymbol{C}_{*}^{1}+\left(1-\chi_{w}(\boldsymbol{x})\right) \boldsymbol{C}\left(\kappa_{0}, \mu_{0}\right)\right]^{-1} \underline{\boldsymbol{\sigma}}_{j}(\boldsymbol{x})\right\rangle \\
\leq W_{f}^{4}\left(\boldsymbol{\sigma}_{1}^{0}, \boldsymbol{\sigma}_{2}^{0}, \boldsymbol{\epsilon}_{1}^{0}, \boldsymbol{\epsilon}_{2}^{0}, \boldsymbol{\epsilon}_{3}^{0}, \boldsymbol{\epsilon}_{4}^{0}\right)+\alpha \epsilon^{\prime}+\beta K / \epsilon^{\prime},
\end{aligned}
$$

where $K$ represents the norm

$$
K=\sqrt{\sum_{i=1}^{4} \delta \boldsymbol{\epsilon}_{i}^{0}: \delta \boldsymbol{\epsilon}_{i}^{0}+\sum_{j=1}^{2} \delta \boldsymbol{\sigma}_{j}^{0}: \delta \boldsymbol{\sigma}_{j}^{0}},
$$


of the field variations. Choosing $\epsilon^{\prime}=\sqrt{\beta K / \alpha}$ to minimize the right-hand side of (9-9), we obtain

$$
\begin{aligned}
W_{f}^{4}\left(\boldsymbol{\sigma}_{1}^{0}+\delta \boldsymbol{\sigma}_{1}^{0}, \boldsymbol{\sigma}_{2}^{0}+\delta \boldsymbol{\sigma}_{2}^{0}, \boldsymbol{\epsilon}_{1}^{0}+\delta \boldsymbol{\epsilon}_{1}^{0}, \boldsymbol{\epsilon}_{2}^{0}+\delta \boldsymbol{\epsilon}_{2}^{0}, \boldsymbol{\epsilon}_{3}^{0}+\delta \boldsymbol{\epsilon}_{3}^{0}, \boldsymbol{\epsilon}_{4}^{0}+\delta \boldsymbol{\epsilon}_{4}^{0}\right) \\
\leq W_{f}^{4}\left(\boldsymbol{\sigma}_{1}^{0}, \boldsymbol{\sigma}_{2}^{0}, \boldsymbol{\epsilon}_{1}^{0}, \boldsymbol{\epsilon}_{2}^{0}, \boldsymbol{\epsilon}_{3}^{0}, \boldsymbol{\epsilon}_{4}^{0}\right)+2 \sqrt{\alpha \beta K} .
\end{aligned}
$$

In obtaining the bound (9-9) we have used the fact that $K^{2}$ is less than $K$ for sufficiently small $K$, specifically $K<1$. Clearly the right-hand side of (9-11) approaches $W_{f}^{4}\left(\boldsymbol{\sigma}_{1}^{0}, \boldsymbol{\sigma}_{2}^{0}, \boldsymbol{\epsilon}_{1}^{0}, \boldsymbol{\epsilon}_{2}^{0}, \boldsymbol{\epsilon}_{3}^{0}, \boldsymbol{\epsilon}_{4}^{0}\right)$ as $K \rightarrow 0$. On the other hand, by repeating the same argument with the roles of

$$
W_{f}^{4}\left(\boldsymbol{\sigma}_{1}^{0}, \boldsymbol{\sigma}_{2}^{0}, \boldsymbol{\epsilon}_{1}^{0}, \boldsymbol{\epsilon}_{2}^{0}, \boldsymbol{\epsilon}_{3}^{0}, \boldsymbol{\epsilon}_{4}^{0}\right)
$$

and

$$
W_{f}^{4}\left(\boldsymbol{\sigma}_{1}^{0}+\delta \boldsymbol{\sigma}_{1}^{0}, \boldsymbol{\sigma}_{2}^{0}+\delta \boldsymbol{\sigma}_{2}^{0}, \boldsymbol{\epsilon}_{1}^{0}+\delta \boldsymbol{\epsilon}_{1}^{0}, \boldsymbol{\epsilon}_{2}^{0}+\delta \boldsymbol{\epsilon}_{2}^{0}, \boldsymbol{\epsilon}_{3}^{0}+\delta \boldsymbol{\epsilon}_{3}^{0}, \boldsymbol{\epsilon}_{4}^{0}+\delta \boldsymbol{\epsilon}_{4}^{0}\right)
$$

reversed, and with

$$
\boldsymbol{C}_{*}\left(\boldsymbol{\sigma}_{1}^{0}+\delta \boldsymbol{\sigma}_{1}^{0}, \boldsymbol{\sigma}_{2}^{0}+\delta \boldsymbol{\sigma}_{2}^{0}, \boldsymbol{\epsilon}_{1}^{0}+\delta \boldsymbol{\epsilon}_{1}^{0}, \boldsymbol{\epsilon}_{2}^{0}+\delta \boldsymbol{\epsilon}_{2}^{0}, \boldsymbol{\epsilon}_{3}^{0}+\delta \boldsymbol{\epsilon}_{3}^{0}, \boldsymbol{\epsilon}_{4}^{0}+\delta \boldsymbol{\epsilon}_{4}^{0}\right)
$$

replacing

$$
\boldsymbol{C}_{*}\left(\boldsymbol{\sigma}_{1}^{0}, \boldsymbol{\sigma}_{2}^{0}, \boldsymbol{\epsilon}_{1}^{0}, \boldsymbol{\epsilon}_{2}^{0}, \boldsymbol{\epsilon}_{3}^{0}, \boldsymbol{\epsilon}_{4}^{0}\right)
$$

we deduce that

$$
\begin{aligned}
& W_{f}^{4}\left(\boldsymbol{\sigma}_{1}^{0}, \boldsymbol{\sigma}_{2}^{0}, \boldsymbol{\epsilon}_{1}^{0}, \boldsymbol{\epsilon}_{2}^{0}, \boldsymbol{\epsilon}_{3}^{0}, \boldsymbol{\epsilon}_{4}^{0}\right) \\
& \leq W_{f}^{4}\left(\boldsymbol{\sigma}_{1}^{0}+\delta \boldsymbol{\sigma}_{1}^{0}, \boldsymbol{\sigma}_{2}^{0}+\delta \boldsymbol{\sigma}_{2}^{0}, \boldsymbol{\epsilon}_{1}^{0}+\delta \boldsymbol{\epsilon}_{1}^{0}, \boldsymbol{\epsilon}_{2}^{0}+\delta \boldsymbol{\epsilon}_{2}^{0}, \boldsymbol{\epsilon}_{3}^{0}+\delta \boldsymbol{\epsilon}_{3}^{0}, \boldsymbol{\epsilon}_{4}^{0}+\delta \boldsymbol{\epsilon}_{4}^{0}\right) \\
& +2 \sqrt{\alpha \beta K} .
\end{aligned}
$$

This, together with (9-11), establishes the continuity of $W_{f}^{4}\left(\boldsymbol{\sigma}_{1}^{0}, \boldsymbol{\sigma}_{2}^{0}, \boldsymbol{\epsilon}_{1}^{0}, \boldsymbol{\epsilon}_{2}^{0}, \boldsymbol{\epsilon}_{3}^{0}, \boldsymbol{\epsilon}_{4}^{0}\right)$. The continuity of the other energy functions follows by the same argument.

\section{Conclusion}

We have established the following two theorems.

Theorem 10.1. Consider composites in three dimensions of two materials with positive definite elasticity tensors $\boldsymbol{C}_{1}$ and $\boldsymbol{C}_{2}=\delta \boldsymbol{C}_{0}$ mixed in proportions $f$ and $1-f$. Let the seven energy functions $W_{f}^{k}$, for $k=0,1, \ldots, 6$, that characterize the set $G U_{f}$ (with $U=\left(\boldsymbol{C}_{1}, \delta \boldsymbol{C}_{0}\right)$ ) of possible elastic tensors be defined by (3-9). These energy functions involve a set of applied strains $\epsilon_{i}^{0}$ and applied stresses $\sigma_{j}^{0}$ meeting the orthogonality condition (3-10). The energy function $W_{f}^{0}$ is given by

$$
W_{f}^{0}\left(\boldsymbol{\sigma}_{1}^{0}, \boldsymbol{\sigma}_{2}^{0}, \boldsymbol{\sigma}_{3}^{0}, \boldsymbol{\sigma}_{4}^{0}, \boldsymbol{\sigma}_{5}^{0}, \boldsymbol{\sigma}_{6}^{0}\right)=\sum_{j=1}^{6} \boldsymbol{\sigma}_{j}^{0}: \widetilde{\boldsymbol{C}}_{f}^{A}\left(\boldsymbol{\sigma}_{1}^{0}, \boldsymbol{\sigma}_{2}^{0}, \boldsymbol{\sigma}_{3}^{0}, \boldsymbol{\sigma}_{4}^{0}, \boldsymbol{\sigma}_{5}^{0}, \boldsymbol{\sigma}_{6}^{0}\right) \boldsymbol{\sigma}_{j}^{0}
$$


(as proved by Avellaneda [1987b]). Here $\widetilde{\boldsymbol{C}}_{f}^{A}\left(\boldsymbol{\sigma}_{1}^{0}, \boldsymbol{\sigma}_{2}^{0}, \boldsymbol{\sigma}_{3}^{0}, \boldsymbol{\sigma}_{4}^{0}, \boldsymbol{\sigma}_{5}^{0}, \boldsymbol{\sigma}_{6}^{0}\right)$ is the effective elasticity tensor of a complementary Avellaneda material that is a sequentially layered laminate with the minimum value of the sum of complementary energies

$$
\sum_{j=1}^{6} \boldsymbol{\sigma}_{j}^{0}: \boldsymbol{C}_{*}^{-1} \boldsymbol{\sigma}_{j}^{0}
$$

Additionally, we now have

$$
\begin{aligned}
& \lim _{\delta \rightarrow 0} W_{f}^{3}\left(\boldsymbol{\sigma}_{1}^{0}, \boldsymbol{\sigma}_{2}^{0}, \boldsymbol{\sigma}_{3}^{0}, \boldsymbol{\epsilon}_{1}^{0}, \boldsymbol{\epsilon}_{2}^{0}, \boldsymbol{\epsilon}_{3}^{0}\right)=\sum_{j=1}^{3} \boldsymbol{\sigma}_{j}^{0}:\left[\widetilde{\boldsymbol{C}}_{f}^{A}\left(\boldsymbol{\sigma}_{1}^{0}, \boldsymbol{\sigma}_{2}^{0}, \boldsymbol{\sigma}_{3}^{0}, 0,0,0\right)\right]^{-1} \boldsymbol{\sigma}_{j}^{0}, \\
& \lim _{\delta \rightarrow 0} W_{f}^{4}\left(\boldsymbol{\sigma}_{1}^{0}, \boldsymbol{\sigma}_{2}^{0}, \boldsymbol{\epsilon}_{1}^{0}, \boldsymbol{\epsilon}_{2}^{0}, \boldsymbol{\epsilon}_{3}^{0}, \boldsymbol{\epsilon}_{4}^{0}\right)=\sum_{j=1}^{2} \boldsymbol{\sigma}_{j}^{0}:\left[\widetilde{\boldsymbol{C}}_{f}^{A}\left(\boldsymbol{\sigma}_{1}^{0}, \boldsymbol{\sigma}_{2}^{0}, 0,0,0,0\right)\right]^{-1} \boldsymbol{\sigma}_{j}^{0}, \\
& \lim _{\delta \rightarrow 0} W_{f}^{5}\left(\boldsymbol{\sigma}_{1}^{0}, \boldsymbol{\epsilon}_{1}^{0}, \boldsymbol{\epsilon}_{2}^{0}, \boldsymbol{\epsilon}_{3}^{0}, \boldsymbol{\epsilon}_{4}^{0}, \boldsymbol{\epsilon}_{5}^{0}\right)=\boldsymbol{\sigma}_{1}^{0}:\left[\widetilde{\boldsymbol{C}}_{f}^{A}\left(\boldsymbol{\sigma}_{1}^{0}, 0,0,0,0,0\right)\right]^{-1} \boldsymbol{\sigma}_{1}^{0}, \\
& \lim _{\delta \rightarrow 0} W_{f}^{6}\left(\boldsymbol{\epsilon}_{1}^{0}, \boldsymbol{\epsilon}_{2}^{0}, \boldsymbol{\epsilon}_{3}^{0}, \boldsymbol{\epsilon}_{4}^{0}, \boldsymbol{\epsilon}_{5}^{0}, \boldsymbol{\epsilon}_{6}^{0}\right)=0 .
\end{aligned}
$$

for all combinations of applied stresses $\boldsymbol{\sigma}_{j}^{0}$ and applied strains $\boldsymbol{\epsilon}_{i}^{0}$. When $\operatorname{det} \boldsymbol{\epsilon}_{1}^{0}=0$ but $\epsilon_{1}^{0}$ is not positive semidefinite or negative semidefinite, we have

$$
\lim _{\delta \rightarrow 0} W_{f}^{1}\left(\boldsymbol{\sigma}_{1}^{0}, \boldsymbol{\sigma}_{2}^{0}, \boldsymbol{\sigma}_{3}^{0}, \boldsymbol{\sigma}_{4}^{0}, \boldsymbol{\sigma}_{5}^{0}, \boldsymbol{\epsilon}_{1}^{0}\right)=\sum_{j=1}^{5} \boldsymbol{\sigma}_{j}^{0}:\left[\widetilde{\boldsymbol{C}}_{f}^{A}\left(\boldsymbol{\sigma}_{1}^{0}, \boldsymbol{\sigma}_{2}^{0}, \boldsymbol{\sigma}_{3}^{0}, \boldsymbol{\sigma}_{4}^{0}, \boldsymbol{\sigma}_{5}^{0}, 0\right)\right]^{-1} \boldsymbol{\sigma}_{j}^{0},
$$

while when the equation $\operatorname{det}\left(\epsilon_{1}^{0}+t \epsilon_{2}^{0}\right)$ has at least two distinct roots for $t$ (the condition for which is given by (7-5)), and additionally, the matrix pencil $\epsilon(t)=$ $\epsilon_{1}^{0}+t \epsilon_{2}^{0}$ does not contain any positive definite or negative definite matrices as $t$ is varied (which requires that the quantities in (7-4) are never all positive, or all negative), we have

$$
\lim _{\delta \rightarrow 0} W_{f}^{2}\left(\boldsymbol{\sigma}_{1}^{0}, \boldsymbol{\sigma}_{2}^{0}, \boldsymbol{\sigma}_{3}^{0}, \boldsymbol{\sigma}_{4}^{0}, \boldsymbol{\epsilon}_{1}^{0}, \boldsymbol{\epsilon}_{2}^{0}\right)=\sum_{j=1}^{4} \boldsymbol{\sigma}_{j}^{0}:\left[\widetilde{\boldsymbol{C}}_{f}^{A}\left(\boldsymbol{\sigma}_{1}^{0}, \boldsymbol{\sigma}_{2}^{0}, \boldsymbol{\sigma}_{3}^{0}, \boldsymbol{\sigma}_{4}^{0}, 0,0\right)\right]^{-1} \boldsymbol{\sigma}_{j}^{0} .
$$

Theorem 10.2. For 2-dimensional composites, the four energy functions $W_{f}^{k}$, for $k=0,1,2,3$, are defined by (6-1), and these characterize the set $G U_{f}$, with $U=$ $\left(\boldsymbol{C}_{1}, \delta \boldsymbol{C}_{0}\right)$, of possible elastic tensors $\boldsymbol{C}_{*}$ of composites of two phases with positive definite elasticity tensors $\boldsymbol{C}_{1}$ and $\boldsymbol{C}_{2}=\delta \boldsymbol{C}_{0}$. These energy functions involve a set of applied strains $\boldsymbol{\epsilon}_{i}^{0}$ and applied stresses $\sigma_{j}^{0}$ meeting the orthogonality condition (3-10). The energy function $W_{f}^{0}$ is given by

$$
W_{f}^{0}\left(\boldsymbol{\sigma}_{1}^{0}, \boldsymbol{\sigma}_{2}^{0}, \boldsymbol{\sigma}_{3}^{0}\right)=\sum_{j=1}^{3} \boldsymbol{\sigma}_{j}^{0}: \widetilde{\boldsymbol{C}}_{f}^{A}\left(\boldsymbol{\sigma}_{1}^{0}, \boldsymbol{\sigma}_{2}^{0}, \boldsymbol{\sigma}_{3}^{0}\right) \boldsymbol{\sigma}_{j}^{0}
$$


(as proved by Avellaneda [1987b]), where $\widetilde{\boldsymbol{C}}_{f}^{A}\left(\boldsymbol{\sigma}_{1}^{0}, \boldsymbol{\sigma}_{2}^{0}, \boldsymbol{\sigma}_{3}^{0}\right)$ is the effective elasticity tensor of a complementary Avellaneda material that is a sequentially layered laminate with the minimum value of the sum of complementary energies

$$
\sum_{j=1}^{3} \boldsymbol{\sigma}_{j}^{0}: \boldsymbol{C}_{*}^{-1} \boldsymbol{\sigma}_{j}^{0}
$$

We also have the trivial result that

$$
\lim _{\delta \rightarrow 0} W_{f}^{3}\left(\boldsymbol{\epsilon}_{1}^{0}, \boldsymbol{\epsilon}_{2}^{0}, \boldsymbol{\epsilon}_{3}^{0}\right)=0 .
$$

When $\operatorname{det} \epsilon_{1}^{0} \leq 0$ we have

$$
\lim _{\delta \rightarrow 0} W_{f}^{1}\left(\boldsymbol{\sigma}_{1}^{0}, \boldsymbol{\sigma}_{2}^{0}, \boldsymbol{\epsilon}_{1}^{0}\right)=\sum_{j=1}^{2} \boldsymbol{\sigma}_{j}^{0}:\left[\widetilde{\boldsymbol{C}}_{f}^{A}\left(\boldsymbol{\sigma}_{1}^{0}, \boldsymbol{\sigma}_{2}^{0}, 0\right)\right]^{-1} \boldsymbol{\sigma}_{j}^{0},
$$

while when $\operatorname{det} \epsilon_{1}^{0}<0$ or when $f(t)=\operatorname{det}\left(\epsilon_{1}^{0}+t \epsilon_{2}^{0}\right)$ is quadratic in $t$ with two distinct roots, or when $f(t)$ is linear in $t$ with a nonzero t coefficient, we have

$$
\lim _{\delta \rightarrow 0} W_{f}^{2}\left(\boldsymbol{\sigma}_{1}^{0}, \boldsymbol{\epsilon}_{1}^{0}, \boldsymbol{\epsilon}_{2}^{0}\right)=\boldsymbol{\sigma}_{1}^{0}:\left[\widetilde{\boldsymbol{C}}_{f}^{A}\left(\boldsymbol{\sigma}_{1}^{0}, 0,0\right)\right]^{-1} \boldsymbol{\sigma}_{1}^{0} .
$$

These theorems, and the accompanying microstructures, help define what sort of elastic behaviors are theoretically possible in 2- and 3-dimensional printed materials. They should serve as benchmarks for the construction of more realistic microstructures that can be manufactured. We have found the minimum over all microstructures of various sums of energies and complementary energies. More realistic designs can be obtained by adding to this sum a term that penalizes the surface area as done for a single energy minimization by Kohn and Wirth [2014; 2016].

It remains an open problem to find expressions for the energy functions in the cases not covered by these theorems. Even for an isotropic composite with a bulk modulus $\kappa_{*}$ and a shear modulus $\mu_{*}$, the set of all possible pairs $\left(\kappa_{*}, \mu_{*}\right)$ is still not completely characterized either in the limit $\delta \rightarrow 0$ or in the limit $\delta \rightarrow \infty$. In these limits the bounds of Berryman and Milton [1988] and Cherkaev and Gibiansky [1993] decouple and provide no extra information beyond that provided by the Hashin-Shtrikman-Hill bounds [Hashin and Shtrikman 1963; Hashin 1965; Hill $1963 ; 1964]$. While the results of this paper show that in the limit $\delta \rightarrow 0$ one can obtain 2- or 3-dimensional structures attaining the Hashin-Shtrikman-Hill upper bound on $\kappa_{*}$, while having $\mu_{*}=0$, it is not clear what the maximum value for $\mu_{*}$ is, given that $\kappa_{*}=0$.

One important corollary of this work is that it gives a complete characterization of the possible triplets $\left(\boldsymbol{\epsilon}^{0}, \boldsymbol{\sigma}^{0}, f\right)$ of average strain $\boldsymbol{\epsilon}^{0}$, average stress $\boldsymbol{\sigma}^{0}$, and volume fraction $f$ that can occur in 2-dimensional and 3-dimensional printed materials in 
the limit $\delta \rightarrow 0$. This will be discussed in a separate paper [Milton and CamarEddine 2016].

\section{Acknowledgements}

The authors thank the National Science Foundation for support through grant DMS1211359. M. Briane wishes to thank the Department of Mathematics of the University of Utah for his stay during March 25-April 3, 2016. Mohamed Camar-Eddine is thanked for initial collaborations that marked the beginning of this work. The authors are grateful to Martin Wegener and his group for allowing them to use one of their electron micrographs of the 3-dimensional pentamode structure, and to Muamer Kadic for help with processing the micrograph.

\section{References}

[Allaire 1994] G. Allaire, "Explicit lamination parameters for three-dimensional shape optimization”, Control Cybernet. 23:3 (1994), 309-326.

[Allaire 2002] G. Allaire, Shape optimization by the homogenization method, Applied Mathematical Sciences 146, Springer, New York, 2002.

[Allaire and Aubry 1999] G. Allaire and S. Aubry, "On optimal microstructures for a plane shape optimization problem”, Struct. Optimiz. 17:2 (1999), 86-94.

[Allaire and Kohn 1993a] G. Allaire and R. V. Kohn, "Optimal bounds on the effective behavior of a mixture of two well-ordered elastic materials", Quart. Appl. Math. 51:4 (1993), 643-674.

[Allaire and Kohn 1993b] G. Allaire and R. V. Kohn, "Explicit optimal bounds on the elastic energy of a two-phase composite in two space dimensions", Quart. Appl. Math. 51:4 (1993), 675-699.

[Allaire and Kohn 1994] G. Allaire and R. V. Kohn, "Optimal lower bounds on the elastic energy of a composite made from two non-well-ordered isotropic materials", Quart. Appl. Math. 52:2 (1994), 311-333.

[Avellaneda 1987a] M. Avellaneda, "Iterated homogenization, differential effective medium theory and applications”, Comm. Pure Appl. Math. 40:5 (1987), 527-554.

[Avellaneda 1987b] M. Avellaneda, "Optimal bounds and microgeometries for elastic two-phase composites”, SIAM J. Appl. Math. 47:6 (1987), 1216-1228.

[Avellaneda and Milton 1989] M. Avellaneda and G. W. Milton, "Bounds on the effective elasticity tensor of composites based on two-point correlations", pp. 89-93 in Composite material technology, 1989: presented at the Twelfth Annual Energy-Sources Technology Conference and Exhibition (Houston, 1989), American Society of Mechanical Engineers, Petroleum Division 24, American Society of Mechanical Engineers, New York, 1989.

[Backus 1962] G. E. Backus, "Long-wave elastic anisotropy produced by horizontal layering", $J$. Geophys. Res. 67:11 (1962), 4427-4440.

[Beran and Molyneux 1966] M. Beran and J. E. Molyneux, "Use of classical variational principles to determine bounds for the effective bulk modulus in heterogeneous media", Q. Appl. Math. 24 (1966), 107-118.

[Berryman and Milton 1988] J. G. Berryman and G. W. Milton, "Microgeometry of random composites and porous media", J. Phys. D 21:1 (1988), 87-94. 
[Bourdin and Kohn 2008] B. Bourdin and R. V. Kohn, "Optimization of structural topology in the high-porosity regime”, J. Mech. Phys. Solids 56:3 (2008), 1043-1064.

[Bückmann et al. 2014] T. Bückmann, M. Thiel, M. Kadic, R. Schittny, and M. Wegener, "An elastomechanical unfeelability cloak made of pentamode metamaterials", Nature Commun. 5 (2014), art. id. 4130, 6 pp.

[Camar-Eddine and Seppecher 2003] M. Camar-Eddine and P. Seppecher, "Determination of the closure of the set of elasticity functionals", Arch. Ration. Mech. Anal. 170:3 (2003), 211-245.

[Cherkaev 2000] A. Cherkaev, Variational methods for structural optimization, Applied Mathematical Sciences 140, Springer, New York, 2000.

[Cherkaev and Gibiansky 1992] A. V. Cherkaev and L. V. Gibiansky, "The exact coupled bounds for effective tensors of electrical and magnetic properties of two-component two-dimensional composites", Proc. Roy. Soc. Edinburgh Sect. A 122:1-2 (1992), 93-125.

[Cherkaev and Gibiansky 1993] A. V. Cherkaev and L. V. Gibiansky, "Coupled estimates for the bulk and shear moduli of a two-dimensional isotropic elastic composite", J. Mech. Phys. Solids 41:5 (1993), 937-980.

[Ciarlet 1989] P. G. Ciarlet, Introduction to numerical linear algebra and optimisation, Cambridge Univ. Press, 1989.

[Francfort and Milton 1994] G. A. Francfort and G. W. Milton, "Sets of conductivity and elasticity tensors stable under lamination", Comm. Pure Appl. Math. 47:3 (1994), 257-279.

[Francfort and Murat 1986] G. A. Francfort and F. Murat, "Homogenization and optimal bounds in linear elasticity", Arch. Rational Mech. Anal. 94:4 (1986), 307-334.

[Francfort et al. 1995] G. Francfort, F. Murat, and L. Tartar, "Fourth-order moments of nonnegative measures on $S^{2}$ and applications”, Arch. Rational Mech. Anal. 131:4 (1995), 305-333.

[Gérard 1989] P. Gérard, "Compacité par compensation et régularité 2-microlocale", exposé VI, 118 in Séminaire sur les Équations aux Dérivées Partielles, 1988-1989, École Polytech., Palaiseau, FR, 1989.

[Gérard 1994] P. Gérard, "Microlocal analysis of compactness", pp. 75-86 in Nonlinear partial differential equations and their applications (Paris, 1991-1993), edited by H. Brezis and J.-L. Lions, Pitman Res. Notes Math. Ser. 302, Longman, Essex, 1994.

[Gibiansky and Cherkaev 1997a] L. V. Gibiansky and A. V. Cherkaev, "Design of composite plates of extremal rigidity", pp. 95-137 in Topics in the mathematical modelling of composite materials, edited by A. Cherkaev and R. Kohn, Progr. Nonlinear Differential Equations Appl. 31, Birkhäuser, Boston, 1997.

[Gibiansky and Cherkaev 1997b] L. V. Gibiansky and A. V. Cherkaev, "Microstructures of composites of extremal rigidity and exact bounds on the associated energy density", pp. 273-317 in Topics in the mathematical modelling of composite materials, edited by A. Cherkaev and R. Kohn, Progr. Nonlinear Differential Equations Appl. 31, Birkhäuser, Boston, 1997.

[Gibiansky and Lakes 1993] L. V. Gibiansky and R. Lakes, "Bounds on the complex bulk modulus of a two-phase viscoelastic composite with arbitrary volume fractions of the components", Mech. Mater. 16:3 (1993), 317-331.

[Gibiansky and Lakes 1997] L. V. Gibiansky and R. S. Lakes, "Bounds on the complex bulk and shear moduli of a two-dimensional two-phase viscoelastic composite", Mech. Mater. 25:2 (1997), 79-95.

[Gibiansky and Milton 1993] L. V. Gibiansky and G. W. Milton, "On the effective viscoelastic moduli of two-phase media, I: Rigorous bounds on the complex bulk modulus", Proc. Roy. Soc. London Ser. A 440:1908 (1993), 163-188. 
[Gibiansky and Sigmund 2000] L. V. Gibiansky and O. Sigmund, "Multiphase composites with extremal bulk modulus", J. Mech. Phys. Solids 48:3 (2000), 461-498.

[Gibiansky et al. 1993] L. V. Gibiansky, R. S. Lakes, and G. W. Milton, "Viscoelastic composites with extremal properties", pp. 369-376 in Structural Optimization 93 (Rio de Janeiro, 1993), World Cong. Opt. Design Struct. Syst. Proc. 1, Fed. Univ. Rio de Janeiro, 1993.

[Gibiansky et al. 1999] L. V. Gibiansky, G. W. Milton, and J. G. Berryman, "On the effective viscoelastic moduli of two-phase media, III: Rigorous bounds on the complex shear modulus in two dimensions", Proc. Roy. Soc. London Ser. A 455:1986 (1999), 2117-2149.

[Grabovsky 1996] Y. Grabovsky, "Bounds and extremal microstructures for two-component composites: a unified treatment based on the translation method", Proc. Roy. Soc. London Ser. A 452:1947 (1996), 919-944.

[Grabovsky 1998] Y. Grabovsky, "Exact relations for effective tensors of polycrystals, I: Necessary conditions", Arch. Rational Mech. Anal. 143:4 (1998), 309-329.

[Grabovsky and Kohn 1995a] Y. Grabovsky and R. V. Kohn, "Microstructures minimizing the energy of a two-phase elastic composite in two space dimensions, I: The confocal ellipse construction”, J. Mech. Phys. Solids 43:6 (1995), 933-947.

[Grabovsky and Kohn 1995b] Y. Grabovsky and R. V. Kohn, "Microstructures minimizing the energy of a two-phase elastic composite in two space dimensions, II: The Vigdergauz microstructure", J. Mech. Phys. Solids 43:6 (1995), 949-972.

[Grabovsky and Sage 1998] Y. Grabovsky and D. S. Sage, "Exact relations for effective tensors of polycrystals, II: Applications to elasticity and piezoelectricity”, Arch. Rational Mech. Anal. 143:4 (1998), 331-356.

[Grabovsky et al. 2000] Y. Grabovsky, G. W. Milton, and D. S. Sage, "Exact relations for effective tensors of composites: necessary conditions and sufficient conditions", Comm. Pure Appl. Math. 53:3 (2000), 300-353.

[Hashin 1962] Z. Hashin, "The elastic moduli of heterogeneous materials", J. Appl. Math. 29:1 (1962), 143-150.

[Hashin 1965] H. Hashin, "On elastic behavior of fibre reinforced materials of arbitrary transverse phase geometry", J. Mech. Phys. Solids 13:3 (1965), 119-134.

[Hashin and Shtrikman 1963] Z. Hashin and S. Shtrikman, "A variational approach to the theory of the elastic behaviour of multiphase materials", J. Mech. Phys. Solids 11:2 (1963), 127-140.

[Hill 1952] R. Hill, "The elastic behaviour of a crystalline aggregate", Proc. Phys. Soc. A 65:5 (1952), 349-354.

[Hill 1963] R. Hill, "Elastic properties of reinforced solids: some theoretical principles", J. Mech. Phys. Solids 11:5 (1963), 357-372.

[Hill 1964] R. Hill, "Theory of mechanical properties of fibre-strengthened materials, I: Elastic behaviour”, J. Mech. Phys. Solids 12 (1964), 199-212.

[Kadic et al. 2012] M. Kadic, T. Bückmann, N. Stenger, M. Thiel, and M. Wegener, "On the practicability of pentamode mechanical metamaterials", Appl. Phys. Lett. 100:19 (2012), art. id. 191901, $5 \mathrm{pp}$.

[Kochmann and Milton 2014] D. M. Kochmann and G. W. Milton, "Rigorous bounds on the effective moduli of composites and inhomogeneous bodies with negative-stiffness phases", J. Mech. Phys. Solids 71 (2014), 46-63.

[Kohn and Lipton 1988] R. V. Kohn and R. Lipton, "Optimal bounds for the effective energy of a mixture of isotropic, incompressible, elastic materials", Arch. Rational Mech. Anal. 102:4 (1988), $331-350$. 
[Kohn and Wirth 2014] R. V. Kohn and B. Wirth, "Optimal fine-scale structures in compliance minimization for a uniaxial load”, Proc. Roy. Soc. A 470:2170 (2014), art. id. 20140432, 16 pp.

[Kohn and Wirth 2016] R. V. Kohn and B. Wirth, "Optimal fine-scale structures in compliance minimization for a shear load”, Comm. Pure Appl. Math. 69:8 (2016), 1572-1610.

[Lipton 1988] R. Lipton, "On the effective elasticity of a two-dimensional homogenised incompressible elastic composite”, Proc. Roy. Soc. Edinburgh Sect. A 110:1-2 (1988), 45-61.

[Lipton 1991] R. Lipton, "On the behavior of elastic composites with transverse isotropic symmetry”, J. Mech. Phys. Solids 39:5 (1991), 663-681.

[Lipton 1992] R. Lipton, "Bounds and perturbation series for incompressible elastic composites with transverse isotropic symmetry", J. Elasticity 27:3 (1992), 193-225.

[Lipton 1994] R. Lipton, "Optimal bounds on effective elastic tensors for orthotropic composites", Proc. Roy. Soc. London Ser. A 444:1921 (1994), 399-410.

[Liu et al. 2007] L. Liu, R. D. James, and P. H. Leo, "Periodic inclusion-matrix microstructures with constant field inclusions", Metal. Mater. Trans. A 38:4 (2007), 781-787.

[Lurie et al. 1982] K. A. Lurie, A. V. Cherkaev, and A. V. Fedorov, "Regularization of optimal design problems for bars and plates, II”, J. Optim. Theory Appl. 37:4 (1982), 523-543.

[Maxwell 1873] J. C. Maxwell, A treatise on electricity and magnetism, I, Clarendon, Oxford, 1873.

[Milton 1980] G. W. Milton, "Bounds on the complex dielectric constant of a composite material", Appl. Phys. Lett. 37:3 (1980), 300-302.

[Milton 1981a] G. W. Milton, "Bounds on the complex permittivity of a two-component composite material", J. Appl. Phys. 52:8 (1981), 5286-5293.

[Milton 1981b] G. W. Milton, "Bounds on the electromagnetic, elastic, and other properties of twocomponent composites", Phys. Rev. Lett. 46:8 (1981), 542-545.

[Milton 1981c] G. W. Milton, "Concerning bounds on the transport and mechanical properties of multicomponent composite materials", Appl. Phys. A 26:2 (1981), 125-130.

[Milton 1985] G. W. Milton, Some exotic models in statistical physics, I: The coherent potential approximation is a realizable effective medium scheme, II: Anomalous first-order transitions (composites, phases, fractals), Ph.D. thesis, Cornell University, 1985, available at http://tinyurl.com/ miltonphd.

[Milton 1986] G. W. Milton, "Modelling the properties of composites by laminates", pp. 150-174 in Homogenization and effective moduli of materials and media (Minneapolis, MN, 1984/1985), edited by J. L. Ericksen et al., The IMA Volumes in Mathematics and its Applications 1, Springer, New York, 1986.

[Milton 1990] G. W. Milton, "On characterizing the set of possible effective tensors of composites: the variational method and the translation method", Comm. Pure Appl. Math. 43:1 (1990), 63-125.

[Milton 1994] G. W. Milton, "A link between sets of tensors stable under lamination and quasiconvexity", Comm. Pure Appl. Math. 47:7 (1994), 959-1003.

[Milton 2002] G. W. Milton, The theory of composites, Cambridge Monographs on Applied and Computational Mathematics 6, Cambridge Univ. Press, 2002.

[Milton and Berryman 1997] G. W. Milton and J. G. Berryman, "On the effective viscoelastic moduli of two-phase media, II: Rigorous bounds on the complex shear modulus in three dimensions", Proc. Roy. Soc. London Ser. A 453:1964 (1997), 1849-1880.

[Milton and Camar-Eddine 2016] G. W. Milton and M. Camar-Eddine, "Complete characterization of the possible (average strain, average stress, volume fraction) triplets that can occur in 3-d printed materials", to be submitted, 2016. 
[Milton and Cherkaev 1995] G. W. Milton and A. V. Cherkaev, "Which elasticity tensors are realizable?", J. Eng. Mater. Tech. 117:4 (1995), 483-493.

[Milton and Kohn 1988] G. W. Milton and R. V. Kohn, "Variational bounds on the effective moduli of anisotropic composites", J. Mech. Phys. Solids 36:6 (1988), 597-629.

[Milton and McPhedran 1982] G. W. Milton and R. C. McPhedran, "A comparison of two methods for deriving bounds on the effective conductivity of composites", pp. 183-193 in Macroscopic properties of disordered media: proceedings of a conference held at the Courant Institute, edited by R. Burridge et al., Lecture Notes in Physics 154, Springer, Berlin, 1982.

[Milton and Phan-Thien 1982] G. W. Milton and N. Phan-Thien, "New bounds on effective elastic moduli of two-component materials", Proc. Roy. Soc. London Ser. A 380:1779 (1982), 305-331.

[Milton et al. 2003] G. W. Milton, S. K. Serkov, and A. B. Movchan, "Realizable (average stress, average strain) pairs in a plate with holes", SIAM J. Appl. Math. 63:3 (2003), 987-1028.

[Milton et al. 2017] G. W. Milton, D. Harutyunyan, and M. Briane, "Towards a complete characterization of the effective elasticity tensors of mixtures of an elastic phase and an almost rigid phase", Math. Mech. Complex Syst. 5:1 (2017), 95-113.

[Nemat-Nasser and Hori 1993] S. Nemat-Nasser and M. Hori, Micromechanics: overall properties of heterogeneous materials, North-Holland Series in Applied Mathematics and Mechanics 37, North-Holland, Amsterdam, 1993.

[Nemat-Nasser and Hori 1998] S. Nemat-Nasser and M. Hori, Micromechanics: overall properties of heterogeneous materials, 2nd ed., Elsevier, Amsterdam, 1998.

[Norris 1985] A. N. Norris, "A differential scheme for the effective moduli of composites", Mech. Mater. 4:1 (1985), 1-16.

[Phani and Hussein 2017] A. S. Phani and M. I. Hussein (editors), Dynamics of lattice materials, Wiley, New York, 2017.

[Roscoe 1973] R. Roscoe, "Isotropic composites with elastic or viscoelastic phases: general bounds for the moduli and solutions for special geometries", Rheol. Acta 12:3 (1973), 404-411.

[Seppecher et al. 2011] P. Seppecher, J.-J. Alibert, and dell'Isola, Francesco, "Linear elastic trusses leading to continua with exotic mechanical interactions", J. Phys. Conf. Ser. 319:1 (2011), art. id. 012018, 13 pp.

[Sigmund 1994] O. Sigmund, "Materials with prescribed constitutive parameters: an inverse homogenization problem”, Internat. J. Solids Structures 31:17 (1994), 2313-2329.

[Sigmund 1995] O. Sigmund, "Tailoring materials with prescribed elastic properties", Mech. Mater. 20:4 (1995), 351-368.

[Sigmund 2000] O. Sigmund, "A new class of extremal composites", J. Mech. Phys. Solids 48:2 (2000), 397-428.

[Tartar 1979] L. Tartar, "Estimation de coefficients homogénéisés", pp. 364-373 in Computing methods in applied sciences and engineering: proceedings of the Third International Symposium (Versailles, 1977), edited by R. Glowinski and J.-L. Lions, Lecture Notes in Mathematics 704, Springer, Berlin, 1979. Translated in Topics Math. Model. Comp. Mater. 31 (1997), 9-20.

[Tartar 1985] L. Tartar, "Estimations fines des coefficients homogénéisés”, pp. 168-187 in Ennio De Giorgi colloquium (Paris, 1983), edited by P. Krée, Research Notes in Mathematics 125, Pitman, Boston, 1985.

[Tartar 1989] L. Tartar, " $H$-measures and small amplitude homogenization", pp. 89-99 in Random media and composites (Leesburg, VA, 1988), edited by R. V. Kohn and G. W. Milton, SIAM, Philadelphia, PA, 1989. 
[Tartar 1990] L. Tartar, " $H$-measures, a new approach for studying homogenisation, oscillations and concentration effects in partial differential equations", Proc. Roy. Soc. Edinburgh Sect. A 115:3-4 (1990), 193-230.

[Tartar 2009] L. Tartar, The general theory of homogenization, Lecture Notes of the Unione Matematica Italiana 7, Springer, Berlin, 2009.

[Torquato 2002] S. Torquato, Random heterogeneous materials: microstructure and macroscopic properties, Interdisciplinary Applied Mathematics 16, Springer, New York, 2002.

[Vigdergauz 1986] S. B. Vigdergauz, "Effective elastic parameters of a plate with a regular system of equal-strength holes", Inzh. Zh. Mekh. Tverd. Tela 21:2 (1986), 165-169. In Russian.

[Vigdergauz 1994] S. Vigdergauz, "Two-dimensional grained composites of extreme rigidity", $J$. Appl. Mech. 61:2 (1994), 390-394.

[Vigdergauz 1996] S. Vigdergauz, "Rhombic lattice of equi-stress inclusions in an elastic plate", Quart. J. Mech. Appl. Math. 49:4 (1996), 565-580.

[Vigdergauz 1999] S. Vigdergauz, "Energy-minimizing inclusions in a planar elastic structure with macroisotropy”, Struct. Optimiz. 17:2 (1999), 104-112.

[Walpole 1966] L. J. Walpole, "On bounds for the overall elastic moduli of inhomogeneous systems, I”, J. Mech. Phys. Solids 14:3 (1966), 151-162.

[Willis 1977] J. R. Willis, "Bounds and self-consistent estimates for the overall properties of anisotropic composites", J. Mech. Phys. Solids 25:3 (1977), 185-202.

[Zhikov 1988] V. V. Zhikov, "Estimates for the trace of an averaged tensor", Dokl. Akad. Nauk SSSR 299:4 (1988), 796-800. In Russian; translated in Soviet Math. Dokl. 37:2 (1988), 456-459.

[Zhikov 1991a] V. V. Zhikov, "Estimates for an averaged matrix and an averaged tensor", Uspekhi Mat. Nauk 46:3(279) (1991), 49-109. In Russian; translated in Russ. Math. Surv. 46:3 (1991), 65136; errata in 47:1 (1992) 278.

[Zhikov 1991b] V. V. Zhikov, "Estimates for the trace of an averaged tensor", Ukrain. Mat. Zh. 43:6 (1991), 745-755. In Russian; translated in Ukrainian Math. J. 43:6 (1992), 694-704.

Received 10 Jun 2016. Revised 11 Oct 2016. Accepted 14 Nov 2016.

GRAEME W. MiLton: milton@math.utah.edu

Department of Mathematics, University of Utah, 155 South 1400 East Room 233,

Salt Lake City, UT 84112-0090, United States

MARC BRIANE: mbriane@insa-rennes.fr

Institut de Recherche Mathématique de Rennes, INSA de Rennes, 20 Avenue des Buttes de Coësmes, CS 70839, 35708 Rennes Cedex 7, France

DAVIT HARUTYUNYAN: davith@math.utah.edu

Department of Mathematics, University of Utah, 155 South 1400 East Room 233,

Salt Lake City, UT 84112-0090, United States 
EDITORIAL BOARD

ANTONIO CARCATERRA

ERIC A. CARLEN

FRANCESCO DELL'ISOLA

RAFFAELE ESPOSITO

ALBERT FANNJIANG

Gilles A. FranCFORT

Pierangelo MARCATI

JEAN-JACQUES MARIGO

PETER A. MARKOWICH

MARTIN OSTOJA-STARZEWSKI

PIERRE SEPPECHER

DAVID J. STEIGMANN

PAUl STEINMANN

PierRe M. SuQueT

MANAGING EDITORS

MICOL AMAR

CORRADO LATTANZIO

ANGELA MADEO

MARTIN OSTOJA-STARZEWSKI

\section{ADVISORY BOARD}

ADNAN AKAY

Holm AltenBaCH

MICOL AMAR

HARM ASKES

TEODOR ATANACKOVIĆ

VICTOR BERDICHEVSKY

GUY BOUCHITTÉ

ANDREA BRAIDES

ROBERTO CAMASSA

MAURO CARFORE

ERIC DARVE

FELIX DARVE

ANNA DE MASI

GianPiEtro DEL Piero

EMMANUELE Di BENEDETTO

BERNOLD FIEDLER

IRENE M. GAMBA

DAVID Y. GAO

SERGEY GAVRILYUK

TIMOTHY J. HEALEY

DOMINIQUE JEULIN

ROGER E. KHAYAT

CORRADO LATTANZIO

ROBERT P. LIPTON

ANGELO LUONGO

ANGELA MADEO

JUAN J. MANFREDI

CARLO MARCHIORO

GÉRARD A. MAUGIN

ROBERTO NATALINI PATRIZIO NEFF

ANDREY PIATNITSKI

ERRICO PRESUTTI

MARIO PULVIRENTI

LUCIO RUSSO

Miguel A. F. SANJUAN

PATRICK SElvadurai

ALEXANDER P. SEYRANIAN

MIROSLAV ŠILHAVÝ

GUIDO SWEERS

ANTOINETTE TORDESILLAS

LEV TRUSKINOVSKY

JUAN J. L. VELÁZQUEZ VINCENZO VESPRI ANGELO VULPIANI msp.org/memocs

Università di Roma “La Sapienza”, Italia

Rutgers University, USA

(CO-CHAIR) Università di Roma "La Sapienza", Italia

(TREASURER) Università dell'Aquila, Italia

University of California at Davis, USA

(CO-CHAIR) Université Paris-Nord, France

Università dell'Aquila, Italy

École Polytechnique, France

DAMTP Cambridge, UK, and University of Vienna, Austria

(CHAIR MANAGING EDITOR) Univ. of Illinois at Urbana-Champaign, USA

Université du Sud Toulon-Var, France

University of California at Berkeley, USA

Universität Erlangen-Nürnberg, Germany

LMA CNRS Marseille, France

Università di Roma "La Sapienza", Italia

Università dell'Aquila, Italy

Université de Lyon-INSA (Institut National des Sciences Appliquées), France

(CHAIR MANAGING EDITOR) Univ. of Illinois at Urbana-Champaign, USA

Carnegie Mellon University, USA, and Bilkent University, Turkey

Otto-von-Guericke-Universität Magdeburg, Germany

Università di Roma "La Sapienza", Italia

University of Sheffield, UK

University of Novi Sad, Serbia

Wayne State University, USA

Université du Sud Toulon-Var, France

Università di Roma Tor Vergata, Italia

University of North Carolina at Chapel Hill, USA

Università di Pavia, Italia

Stanford University, USA

Institut Polytechnique de Grenoble, France

Università dell'Aquila, Italia

Università di Ferrara and International Research Center MEMOCS, Italia

Vanderbilt University, USA

Freie Universität Berlin, Germany

University of Texas at Austin, USA

Federation University and Australian National University, Australia

Université Aix-Marseille, France

Cornell University, USA

École des Mines, France

University of Western Ontario, Canada

Università dell' Aquila, Italy

Louisiana State University, USA

Università dell'Aquila, Italia

Université de Lyon-INSA (Institut National des Sciences Appliquées), France University of Pittsburgh, USA

Università di Roma "La Sapienza”, Italia

Université Paris VI, France

Istituto per le Applicazioni del Calcolo "M. Picone", Italy

Universität Duisburg-Essen, Germany

Narvik University College, Norway, Russia

Università di Roma Tor Vergata, Italy

Università di Roma "La Sapienza”, Italia

Università di Roma “Tor Vergata”, Italia

Universidad Rey Juan Carlos, Madrid, Spain

McGill University, Canada

Moscow State Lomonosov University, Russia

Academy of Sciences of the Czech Republic

Universität zu Köln, Germany

University of Melbourne, Australia

École Polytechnique, France

Bonn University, Germany

Università di Firenze, Italia

Università di Roma La Sapienza, Italia

MEMOCS (ISSN 2325-3444 electronic, 2326-7186 printed) is a journal of the International Research Center for the Mathematics and Mechanics of Complex Systems at the Università dell'Aquila, Italy.

Cover image: "Tangle” by $\odot$ John Horigan; produced using the Context Free program (contextfreeart.org).

PUBLISHED BY

7 mathematical sciences publishers

nonprofit scientific publishing

http://msp.org/

(C) 2017 Mathematical Sciences Publishers 
Mathematics and Mechanics of Complex Systems vol. 5 no. 1

Reducible and irreducible forms of stabilised gradient

elasticity in dynamics

Harm Askes and Inna M. Gitman

Dating Hypatia's birth: a probabilistic model

Canio Benedetto, Stefano Isola and Lucio Russo

On the possible effective elasticity tensors of 2-dimensional 41 and 3-dimensional printed materials

Graeme W. Milton, Marc Briane and Davit Harutyunyan Towards a complete characterization of the effective elasticity tensors of mixtures of an elastic phase and an almost rigid phase

Graeme W. Milton, Davit Harutyunyan and Marc Briane

MEMOCS is a journal of the International Research Center for the Mathematics and Mechanics of Complex Systems at the Università dell' Aquila, Italy.

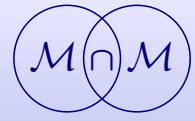

